\title{
ASYNCHRONOUS MIPS PROCESSORS: EDUCATIONAL SIMULATIONS
}

\author{
A Thesis \\ Presented to \\ the Faculty of California Polytechnic State University \\ San Luis Obispo
}

\author{
In Partial Fulfillment \\ of the Requirements for the Degree \\ Master of Science in Computer Science
}

by

Robert Webb

July 2010 
(C) 2010

Robert Webb

ALL RIGHTS RESERVED 


\section{COMMITTEE MEMBERSHIP}

TITLE: Asynchronous MIPS Processors: Educational Simulations

AUTHOR: $\quad$ Robert Webb

DATE SUBMITTED: July 2010

COMMITTEE CHAIR: Chris Lupo, Ph.D.

COMMITTEE MEMBER: Christopher Clark, Ph.D.

COMMITTEE MEMBER: Phillip Nico, Ph.D. 


\section{Abstract \\ Asynchronous MIPS Processors: Educational Simulations \\ Robert Webb}

The system clock has been omnipresent in most mainstream chip designs. While simplifying many design problems the clock has caused the problems of clock skew, high power consumption, electromagnetic interference, and worst-case performance. In recent years, as the timing constraints of synchronous designs have been squeezed ever tighter, the efficiencies of asynchronous designs have become more attractive. By removing the clock, these issues can be mitigated. However, asynchronous designs are generally more complex and difficult to debug. In this paper I discuss the advantages of asynchronous processors and the specifics of some asynchronous designs, outline the roadblocks to asynchronous processor design, and propose a series of asynchronous designs to be used by students in tandem with traditional synchronous designs when taking an undergraduate computer architecture course. 


\section{Acknowledgements}

Thank you to my parents... ${ }^{1}$

${ }^{1}$...for paying my tuition, among other things. 


\section{Contents}

List of Figures $\quad$ ix

1 Introduction 1

1.1 Advantages of Asynchronous Design . . . . . . . . . . . . . 2

1.1.1 Potential Speed Increase . . . . . . . . . . . . . . . 2

1.1.2 Elimination of Clock Skew . . . . . . . . . . . . . 2

1.1.3 Electromagnetic Interference . . . . . . . . . . . 3

1.1.4 Reduced Power Consumption ... . . . . . . . . 3

1.2 Roadblocks ......................... 3

1.2.1 Design Difficulties . . . . . . . . . . . . . . . . 4

1.2.2 Design Tools................... 4

1.2.3 Lack of Academic Courses . . . . . . . . . . . . . 4

1.3 Outline: Parallels to

Computer Organization and Design . . . . . . . . . . 5

2 Previous Work $\quad 7$

2.1 Asynchronous Logic . . . . . . . . . . . . . . . . 7

2.2 Early Developments . . . . . . . . . . . . . . . 8

2.3 Modern Developments . . . . . . . . . . . . . . 8

2.4 Educational Resources . . . . . . . . . . . . . . . . . . . . 10

3 A Single Cycle Asynchronous MIPS Processor 11

3.1 Design Principles . . . . . . . . . . . . . . . 11

3.1.1 Dual Rail Encoding . . . . . . . . . . . . . . . 11

3.1 .2 Dual Rail Logic . . . . . . . . . . . . . . . . . . . . . . . 12

3.2 Simulation . . . . . . . . . . . . . . . . . . . 14 
3.2.1 The Synchronous Starting Point . . . . . . . . . . . . . . 14

3.2.2 Beginning the Asynchronous Conversion . . . . . . . . . 15

3.2 .3 Completion Signals . . . . . . . . . . . . . . . . 16

3.2 .4 Clock Removal . . . . . . . . . . . . . . . . . . . 19

3.2.5 Distribution of the GCS . . . . . . . . . . . 20

3.3 Results and Discussion . . . . . . . . . . . . . . . 20

3.3.1 Design Lessons . . . . . . . . . . . . . . . . . . . 20

3.3 .2 Results. . . . . . . . . . . . . . . . . . . 22

4 An Asynchronous Pipelined MIPS Processor 23

4.1 Theory . . . . . . . . . . . . . . . . . . . . . 23

4.1 .1 Delay Insensitivity $\ldots \ldots$

4.1 .2 Quasi-Delay Insensitivity . . . . . . . . . . . . . . . . 24

4.1 .3 The Muller C Element . . . . . . . . . . . . . . . . . 24

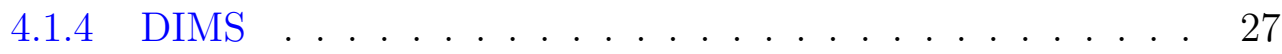

4.1.5 Design Choices . . . . . . . . . . . . . . . . 30

$4.1 .6 \quad$ Muller Pipeline . . . . . . . . . . . . . . . . . . 33

4.2 Simulation . . . . . . . . . . . . . . . . . . . . . 39

4.2 .1 Data Path Decomposition . . . . . . . . . . . . . 39

$4.2 .2 \quad \mathrm{ALU} \ldots \ldots \ldots \ldots \ldots \ldots \ldots \ldots$

4.3 Results . . . . . . . . . . . . . . . . . . . . . 45

5 Educational Resources $\quad 47$

5.1 Laboratory Exercises . . . . . . . . . . . . . . . . . 47

5.2 Notes on the use of these simulations . . . . . . . . . . . 49

6 Conclusions and Future Work $\quad 50$

6.1 Conclusion . . . . . . . . . . . . . . . . . . 50

6.2 Future Work . . . . . . . . . . . . . . . . . . . . 51

Bibliography $\quad 52$

A The Complete Synchronous Single-Cycle Source Code 54

B The Complete Single-Cycle Asynchronous Source Code $\quad 70$

C The Complete Pipelined Asynchronous Source Code 89 
D Laboratory Exercises for students

114

viii 


\section{List of Figures}

3.1 basic dual rail NOT gate: NOT2 . . . . . . . . . . . . . 12

3.2 basic dual rail AND gate: AND2 . . . . . . . . . . . . 13

3.3 basic dual rail OR gate: OR2 . . . . . . . . . . . . . . . . 13

3.4 Completion Detector . . . . . . . . . . . . . . . 18

3.5 The single cycle asynchronous processor excluding control . . . . . 21

4.1 C element: gate description and transistor implementation . . . 26

4.2 C Element minterms for a 2 input DIMS circuit . . . . . . . . . . 27

4.3 DIMS AND gate . . . . . . . . . . . . . . . 28

4.4 DIMS OR gate . . . . . . . . . . . . . . . . . . . . . 29

4.5 Conversion Unit, Bundled Data into Dual-rail . . . . . . . . . . 31

4.6 Conversion Unit, Dual-rail into Bundled Data . . . . . . . . . . . 32

4.7 The 4 phase handshake protocol waveform . . . . . . . . . . . . . 34

4.8 A basic asynchronous pipeline made from pipeline control units . 35

4.9 Muller Pipeline Unit . . . . . . . . . . . . . . . . . . . . . . 35

4.10 A basic asynchronous pipeline with latches . . . . . . . . . . 36

4.11 Dual-Rail Pipeline Unit . . . . . . . . . . . . . . . . . . 36

4.12 Dual-Rail Pipeline Gate with Multiple data Inputs . . . . . . . . 37

4.13 A basic asynchronous pipeline with a constant delay processing unit 38

4.14 A basic asynchronous pipeline with a variable delay processing unit 38

4.15 Instruction Fetch Stage with control for jump commands . . . . . 40

4.16 Instruction Decode Stage with control and branch decision . . . . 41

4.17 Execute Stage with wired for Memory Bypass . . . . . . . . . . . 43 
4.18 DIMS Adder with Generate and Kill carry . . . . . . . . . . . . . 44 


\section{Chapter 1}

\section{Introduction}

Computer chip designers have long relied on a system wide clock to signal the change of a state. This has resulted in designs where all instructions are assumed to introduce the worst possible delay. This bottleneck has been mitigated by solutions such as pipelining, branch prediction, and dynamic scheduling. However, the recent slowdown in the progress of designers to further increase the clock rates of chips has led to renewed interest in designing a processor without a clock.

Using an internal clock requires that a chip can only progress as fast as the slowest component, which has created increasingly complex pipelines. Even with these very complex pipelines, much of the chip is inactive for a large portion of the time between cycles. Also, as clock rates increase, the size of the circuits has become an issue because of the need for the clock pulse to reach every component of the chip before the next pulse occurs.

Asynchronous design is a possible solution to many of these roadblocks. This thesis presents the basic theory of asynchronous circuit design. To further demonstrate these ideas, simulations of asynchronous processors are introduced. The simulations are designed in parallel with popular synchronous designs to facilitate their use as pedagogical tools, as the lack of academic resources has been one of the principal reasons asynchronous designs have not reached more widespread awareness. 
The available literature does not contain a small simulation of a working asynchronous processor with laboratory exercises. The primary contribution of this paper is to fill that gap. The simulations detailed here are small and easily accessible. The first could be introduced in a few additional lectures once a single cycle synchronous design has been discussed. Depending on the detail desired, introducing the pipelined design to a class could take anywhere from a few days to a few weeks of lecture and lab.

\subsection{Advantages of Asynchronous Design}

The advantages of asynchronous designs can be categorized primarily in terms of speed increase, elimination of the clock skew problem, reduction of electromagnetic interference, and lower power consumption.

\subsubsection{Potential Speed Increase}

A processor that does not have a clock can proceed when processing is complete. There is no need to wait for the next synchronizing clock cycle. For example, when using a simple cascading adder, the addition of two small numbers is fast. However, the subtraction of two small numbers takes quite a bit of time because the two's complement representation of negative numbers requires that all of the significant bits be determined in a cascade. The ALU pipeline stage must be long enough for the worst possible case, but an asynchronous processor can move on once the calculation is completed.

\subsubsection{Elimination of Clock Skew}

As clock rates have become faster the speed of signals across the distance of the chip has become a problem. The maximum delay of the clock signal across the chip is the clock skew, and this delay must be small when compared 
to the frequency of the clock. In modern designs methods of clock distribution have become increasingly complicated and expensive in order to mitigate this problem.[12] Creating processors without a clock eliminates this problem.

\subsubsection{Electromagnetic Interference}

The clock pulse must be powerful enough to reach every part of the chip to synchronize operations. This powerful electric current traveling through the chip can cause significant electromagnetic interference for other nearby components. Eliminating the clock significantly reduces electromagnetic interference produced by processors because the total current will be lower and it will not pulse at a constant frequency.[12]

\subsubsection{Reduced Power Consumption}

Am important commercial interest in asynchronous processors has been their extremely low power consumption. The largest power requirement of a processor is the clock signal, which must occur even when the processor is idle [12]. An asynchronous processor never has to generate a clock signal, so uses less power when running. Also, upon a cache miss or other event requiring the processor to wait for other components of the system, the processor can maintain an idle state with essentially no power consumption. Because of these factors, an asynchronous design consumes significantly less power than synchronous processors. This makes an asynchronous processor an ideal candidate for a battery powered mobile device.

\subsection{Roadblocks}

There are several roadblocks to producing asynchronous processors. These stem from the increased difficulty of asynchronous design and the inertia of industry and educational institutions in the direction of synchronous designs. Specif- 
ically, there are few design tools, trained engineers, or academic courses on the subject.

\subsubsection{Design Difficulties}

The lack of a synchronizing clock makes design much more difficult. Every part of the processor is acting independently, which can lead to unexpected interactions and behavior. Fixing the problems with one set of interacting systems can cause cascading problems down the pipeline.

\subsubsection{Design Tools}

The few design tools that are available to engineers have not been widely adopted. Also, when compared to existing synchronous tools, the asynchronous design tools automate fewer parts of the design process and do not provide as much framework for debugging and testing. For more information on available tools see [1].

\subsubsection{Lack of Academic Courses}

Very few schools offer any kind of academic training in asynchronous design. This has led to most engineers being unaware of the theory that has been developed by research groups developing asynchronous designs. Because the theories have not been widely taught, asynchronous design problems can seem more daunting [12]. 


\subsection{Outline: Parallels to}

\section{Computer Organization and Design}

The purpose of this paper is to propose a way of integrating asynchronous design into a traditional undergraduate computer architecture class using Patterson and Hennessy's "Computer Organization and Design" (COD) [11] as a starting point. The project began with a term paper at Cal Poly in Winter 2009 with Dr. John Seng. It was impractical to learn all the theory of asynchronous design in the timespan of the 10 week quarter, so the goal was to implement a basic single cycle asynchronous processor using mostly synchronous design principals from COD while borrowing asynchronous principals only when necessary. In the end, only dual rail encoding and basic dual rail gates (defined in section 3.1) were required. However, several timing assumptions were required to make the simulation simple enough to complete in the time allotted. The result was a design that was not robust.

To expand the term project into a master's thesis, pipelining and the necessary theory to develop a robust design would be added. In order to integrate the work into existing courses, the design would continue to parallel COD. The final product would be a set of asynchronous simulations that could be used in a traditional course focused on synchronous designs to demonstrate asynchronous designs.

To parallel the machine arithmetic chapter of COD, two asynchronous ALU units are presented. The first is naive, but easy to understand, and presented in section 3.2.2. The second requires more theory, but is much more robust in that it has fewer timing assumptions, and the timing assumptions are mostly local in nature. The theory required to understand the second ALU is presented in section 4.1. The ALU design is presented in section 4.2.2.

To parallel the single cycle data-path chapter of COD, a simple asynchronous processor is presented. This design was created using the design from the text and converting the data path wires to dual-rail encoding and any logic involving 
these wires to dual-rail gates. These busses are then used to create completion signals which take the place of the clock. As noted above, to make this simulation work, several naive assumptions needed to be made. This design is the subject of chapter 3 .

To parallel the pipelining chapter of COD, a pipelined processor using asynchronous design techniques is presented. This is presented in chapter 4 . The chapter includes a detailed description of the theory that was incorporated into the design in section 4.1. The design of the simulation is detailed in section 4.2.

This report is concluded with a description of the educational resources that accompany the project in chapter 5 and appendix D and a discussion of conclusions and possible future research in chapter 6 . Also included as appendices are the complete source code for the single-cycle synchronous processor (appendix A), single-cycle asynchronous processor (appendix B), pipelined asynchronous processor (appendix C). 


\section{Chapter 2}

\section{Previous Work}

\section{$2.1 \quad$ Asynchronous Logic}

For small scale circuits the cumulative effects of gate and wire delay are not difficult to deal with. For this reason, many small circuits do not have a clock because one is simply not needed. The fact that these circuits were asynchronous is generally not explicitly stated. For a discussion of these circuits, see chapter 8 of [14].

When the number of switches or transistors in a circuit began to grow exponentially, quickly approaching the millions, the problem of synchronizing the behavior of a circuit became nontrivial. This was exacerbated by the problem that these pioneers were literally manufacturing the first computers, so all of these circuits had to be laid out by hand on paper. Because CAD simulations were not available, the solution that most designers used was to determine an upper bound on the worst case delay of a circuit or segment of a circuit and use an external clock to signal the completion of the logic and stability of the outputs. 


\section{$2.2 \quad$ Early Developments}

An exception to this trend toward synchronous designs were the designers of ILLIAC II at the University of Illinois in the late 1950's and early 1960's [10]. One of the first pipelined computers, it also had many components designed to work without an external clock. Much of the early theory of large scale asynchronous circuits was developed by the designers of ILLIAC II, specifically David E. Muller. Because, at the time, wire delay was insignificant compared to gate delay, a theory of "Speed-Independent" (SI) circuits was developed to generate logic that would not glitch given arbitrary gate delay. A SI circuit is assumed to have no wire delay. A new gate was developed to accomplish this, the Muller C-Element [10]. These developments are discussed in detail in section 4.1. The handshaking protocols used in the pipelined simulation were also developed in this period by Muller. This remained the standard handshaking protocol until 1988 when Ivan Sutherland proposed an improvement in [13], but this improvement is not used here because of the intended audience and additional circuit complexity.

\subsection{Modern Developments}

The principal inspiration for this project has been the work of the Asynchronous Processor Group at the California Institute of Technology, led by Alain J. Martin. One of the early contributions of this research group was the paper that described the limitations of the successor to SI circuit theory, "Delay-Insensitive" (DI) circuit theory.

As manufacturing processes improved to include more complex circuits with faster switching, it became clear that wire delay and gate delay were concurrent problems. This led to the development a theory of DI circuits where the delay of wires AND gates could be any arbitrary non-negative value without causing any unstable outputs. In the early 80's, Martin developed a model for circuit timing similar to the context-free languages used in computability theory and language theory to prove that any circuit conforming to the requirements of DI would only 
be functionally equivalent to a circuit made entirely from C-elements. As the C-element is not a universal gate ${ }^{1}$, this creates a very limited set of potential DI circuits [7].

The limitations led to the development of a compromise to the strict requirements of DI. Called "Quasi-Delay Insensitive" (QDI), nearly all asynchronous development has followed this paradigm. The compromise is to simply allow a wire to fork out locally and guarantee that the data carried on that wire will reach all the local receivers at the same time. These are called "iso-chronic" forks. Manohar and Martin proved that these circuits are Turing-complete in 1995. [2] Because of the limitations of the original definition of DI and the adoption of QDI, most modern references to DI are, in fact, referring to the assumptions and requirements of QDI.

In the 80's, Cal Tech also developed the first QDI general purpose processor, mostly as a proof-of-concept. A RISC ${ }^{2}$ design, it was finely pipelined and employed many opportunities for concurrent processing that would have been significantly more difficult in a synchronous design. Most significantly, the execute, memory, and write back stages of a traditional 5 stage pipeline were designed to run in parallel, asynchronously. Specifically, there were several busses to write back into the register file, and different instructions could simultaneously be accessing the ALU and memory modules, while asynchronously writing back to the registers. Results were promising, even with manufacturing defects. Most impressively, the processor was entirely QDI. This allowed it to run at a wide range of voltages and temperatures $[3,4]$.

The advancements in asynchronous design led to speculation that clockless processors were going to be much more widely used in the 90's [5]. While this speculation proved incorrect, progress in asynchronous design continued. Martin published the design of a fast asynchronous adder in 1991 [6]. This design was optimized by going down to the transistor level, so was not used in this project as the intended audience for the simulation is computer science students, not

\footnotetext{
${ }^{1} \mathrm{C}$-elements cannot be used to create a circuit that is equivalent to all the other standard logic gates.

${ }^{2}$ Reduced Instruction Set Computing
} 
electrical engineers, however, it could significantly increase the logic speed of asynchronous circuits. For a discussion of several asynchronous adders, including the one detailed in section 4.2.2 and the one designed by Martin, see section 5.3 of $[12]$.

As an additional proof-of-concept, Martin's group produced an asynchronous processor running the MIPS R3000 machine language [8, 9]. This processor is currently the state of the art in terms of asynchronous design and the next step in the logical development after [3]. The papers also developed a new metric for the performance of asynchronous processors, $E \tau^{2}$ where $E$ is the average energy per instruction and $\tau$ is the average instruction execution time. Experiments suggested that, for a given design, that $E \tau^{2}$ is roughly constant because voltage varies inversely with the square of speed.

\subsection{Educational Resources}

As asynchronous design has been "the next big thing" and "just around the corner" for quite some time, it has been only recently that the subject has been the subject of publications beyond short journal articles or technical reports. The lack of textbooks has been a roadblock to the teaching of asynchronous design because most available material was not very student-friendly. Two monographs have been written to remedy this. Davis and Nowick wrote a circuit design manual in 1997 is targeted more toward the experienced designer [1]. Sparsøwrote a textbook targeted to graduate students and advanced undergraduates in 2006 that is freely available on his web page [12]. This textbook has been invaluable to this project. It was the primary reference, especially for pipeline design, even though the principle pipeline control design dates back to Muller and Illiac II. As noted in section 1, these books do not include a small, student accessible, simulation of an asynchronous processor. 


\section{Chapter 3}

\section{A Single Cycle Asynchronous}

\section{MIPS Processor}

This chapter describes a single cycle asynchronous MIPS processor that closely follows the designs presented in COD. To make the simulation accessible, asynchronous circuit theory is used as little as possible. The result is that the processor is simple, but not robust.

\subsection{Design Principles}

\subsubsection{Dual Rail Encoding}

In dual rail encoding every bit $A$ is represented by a pair of bits $A H$ and $A L$, for $A$-high and $A$-low. If $A=0$ then $A H=0$ and $A L=1$. If $A=1$ then $A H=1$ and $A L=0$. If $A$ has not been fully determined, then $A H=0$ and $A L=0$. The situation where $A H=A L=1$ has different meanings in each of the simulations. For the first simulation, this case will also imply that the bit is undetermined. For the more advanced pipeline simulation, the circuit will be designed so that this case cannot occur. See section 4.1.4 for more on this more 


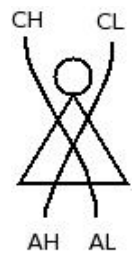

Figure 3.1: basic dual rail NOT gate: NOT2

advanced paradigm.

\subsubsection{Dual Rail Logic}

All the logic gates used in the traditional encoding of data have an equivalent gate that takes dual rail data as input and produces dual rail data as output.

$$
\overline{(A H, A L)}=(A L, A H)
$$

so $\overline{(1,0)}=(0,1)$ and $\overline{(0,1)}=(1,0)$ as shown in figure 3.1. If $A$ has not yet been determined then $\bar{A}$ will not be determined either

$$
\overline{(0,0)}=(0,0)
$$

Similarly

$$
(A H, A L) \wedge(B H, B L)=(A H \wedge B H, A L \vee B L)
$$

as shown in figure 3.2 and

$$
(A H, A L) \vee(B H, B L)=(A H \vee B H, A L \wedge B L)
$$

as shown in figure 3.3.

These new gates are referred to as NOT2, AND2, and OR2 in the simulations using these constructions.

As can be seen, if both inputs to either AND2 or OR2 are undetermined then the output is undetermined. If one of the inputs to AND2 is low then it will output low, even if the other is undetermined. If one of the inputs to AND2 is high then it will output undetermined if the other input is undetermined. If 


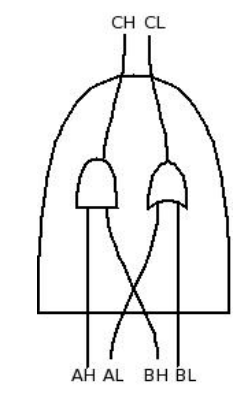

Figure 3.2: basic dual rail AND gate: AND2

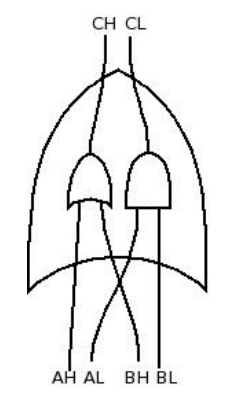

Figure 3.3: basic dual rail OR gate: OR2

one of the inputs to OR2 is high then it will output high, even if the other is undetermined. If one of the inputs to OR2 is low then it will output undetermined if the other input is undetermined.

The fact that these new "gates" are actually more complicated circuits constructed from traditional gates implies that timing is still a concern. For example, if the AND gate inside the AND2 is slower than the OR gate inside AND2, then the output of the AND2 will produce an output of $(1,1)$ when transitioning from an output of $(1,0)$ to an output of $(0,1)$. This is an example of a "glitch". Because of this problem an output of $(1,1)$ is defined as undetermined, and XOR is used to determine if the output of a circuit using these gates is ready to be used. While this method is used in the first simulation, a more sophisticated construction is used in the second simulation that will keep these "glitches" from occurring. This construction requires more theory to be developed which is detailed in 4.1.4. 


\subsection{Simulation}

Because the target audience of this simuation is an undergraduate studying COD, a simple single stage abbreviated MIPS simulator has been altered to incorporate asynchronous design principles.

\subsubsection{The Synchronous Starting Point}

The first design is a single state synchronous MIPS simulation capable of using the addi (add immediate), add (add register), sw (save word to memory), 1w (load word from memory), $\mathrm{j}$ (jump), and bne (branch when not equal) instructions. A simple instruction memory, and data memory have been simulated using behavioral Verilog. ${ }^{1}$ An ALU was constructed used structural Verilog code with the naive assumption that all AND, NOT, and OR gates generate one time unit of delay. The adder is a simple cascading design. A simple program has been written to test the functioning of the unit.

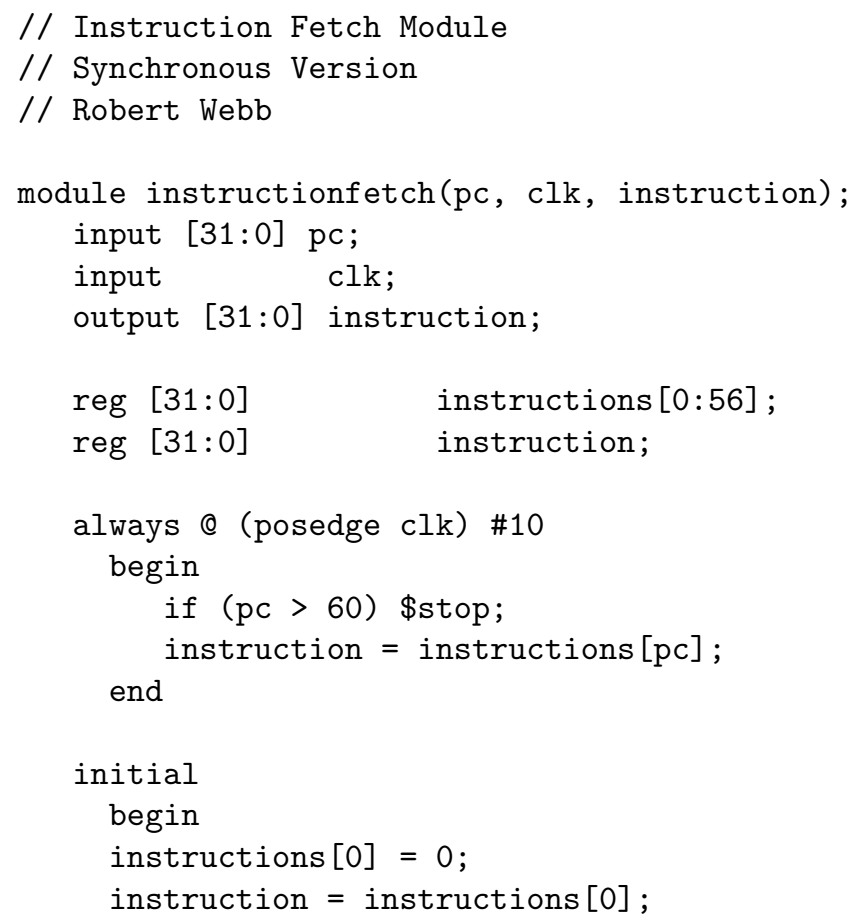

${ }^{1}$ Behavioral Verilog is a specification of the behavior of a circuit without specifying the physical design of the circuit. 


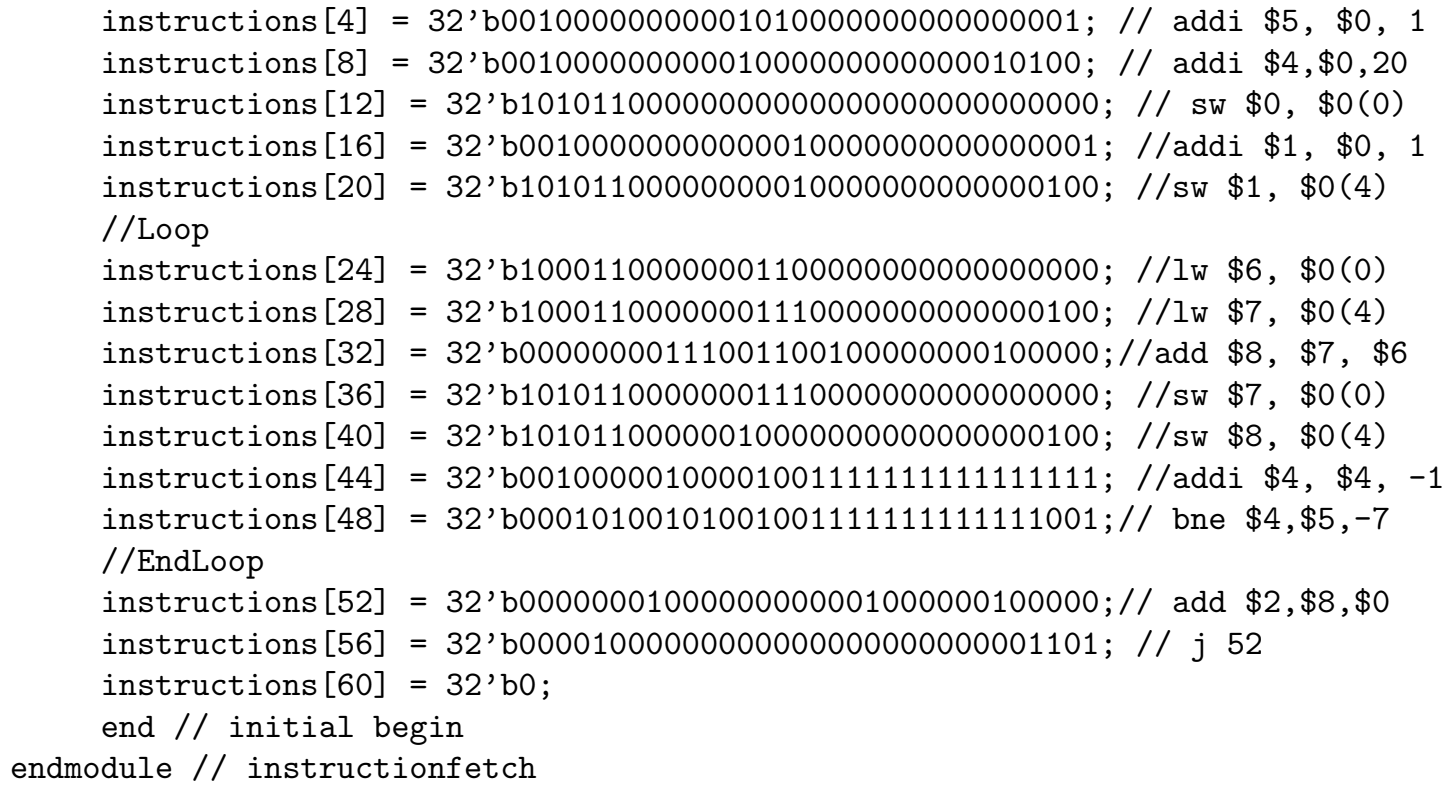

At the conclusion of this program register $\$ 2$ contains the $20^{\text {th }}$ Fibonacci number 6765 . The remainder of the code for the traditional synchronous processor is presented as an appendix.

With the previously stated assumptions, the above program will run with a clock frequency of 96 time units per cycle. At this speed the simulation completes in 13488 time units. These values are noted so they can be compared to the speed of the asynchronous designs.

\subsubsection{Beginning the Asynchronous Conversion}

Verilog is a modular design tool, so the logical starting point is to define new modules corresponding to dual rail AND, OR, and NOT. Because NOT2 has no logical components, it was assumed to have no delay.

For AND2 and OR2 to work properly, delay must be introduced so the undetermined bits will be logically obvious. Assuming that AND took one tick to transition up and two ticks to transition down while OR instantaneously transitioned up and took one tick to transition down produced the desired behavior. These delays were determined by running the simulation with different delays 
while observing the outputs of the ALU. Using these delays, the undetermined bits cascaded down the outputs of the ALU as the carry rippled through the circuit, while guaranteeing that there was always an undetermined bit until all bits were defined. All the busses were then converted to dual rail encoding and all logical gates were converted to dual rail gates.

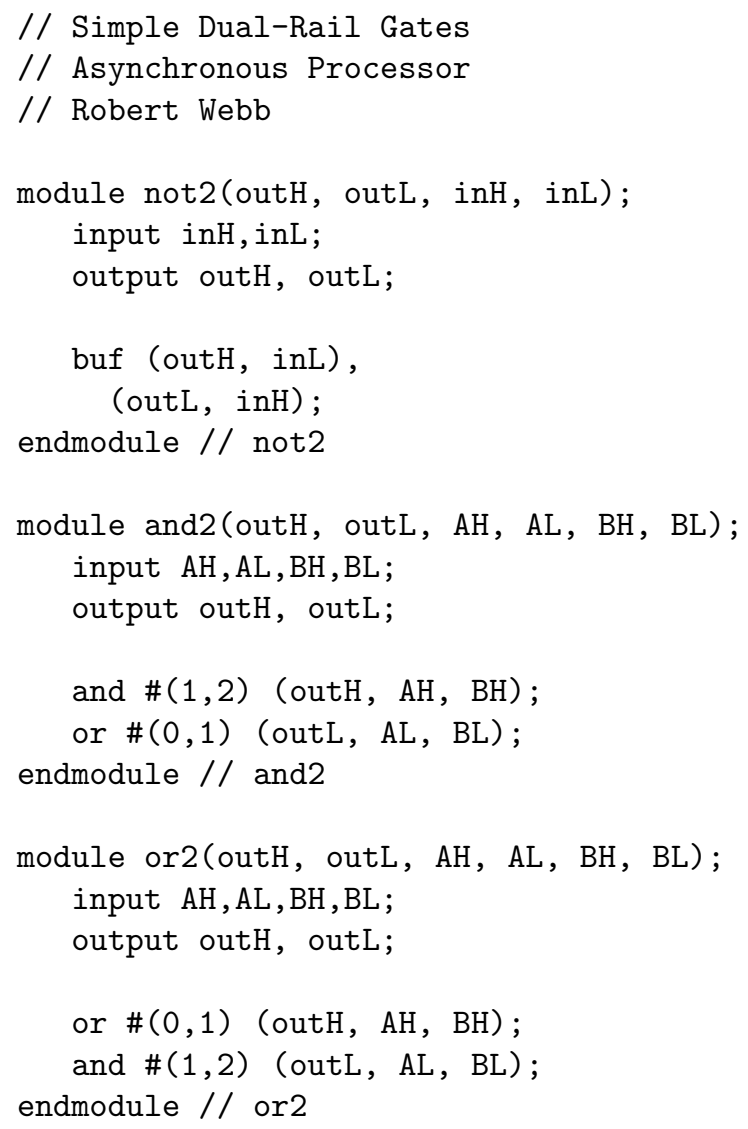

\subsubsection{Completion Signals}

The only parts of the processor that introduce significant and non-constant delay in this simulation are the $3 \mathrm{ALU}$ units. The first ALU increments the current PC by 4 . The second ALU increments the incremented PC by the signextended and shifted immediate from the current instruction. The completion signals from these units are combined to generate a signal referred to as PCready in the simulation specification. The final ALU performs the calculation required by the instruction. The completion signal from this unit is referred to as ALUready 
in the simulation specification. To determine if an ALU has completed it's work, a module in Verilog is used. The basic circuit is shown in figure 3.4.

Depending on the situation, all three may need to be finished to move on. If the instruction is not a branch, then the ALU that is computing branchPC is computing garbage, so the ALU adding the shifted, sign extended immediate and the incremented PC does not need to be finished. The simulated processor does take advantage of this fact in that the PCready signal is computed using the output of the multiplexor that selects nextPC. This allows PCready to signal without waiting for branchPC if it is not needed.

The completion detector module takes a high and low output from an ALU or other processing unit ${ }^{2}$ and returns a high bit if it has completed its work and a low bit if it has not. The determination is accomplished by computing the XOR of each high and low bits and then taking the AND of all of these (called good in the code). A BUF with a delay of 3 time units is then used to delay the output of the AND (called really in the code) which is then compared to the real-time output of the AND gate. The delayed signal is used to attenuate any "glitches" in the processor that might "jump the gun". Attenuation is accomplished because BUF module in Verilog is internally defined to ignore any signals that do not remain high for the duration of the delay of the module. These signals are then passed through a behavioral bit of code that simply changes the possible bit value of $x$ that is produced by Verilog at the beginning of the simulation into a 0 . The behavioral code is only used to get the process started at the beginning because none of the processing units will initiate with an undefined completion signal.

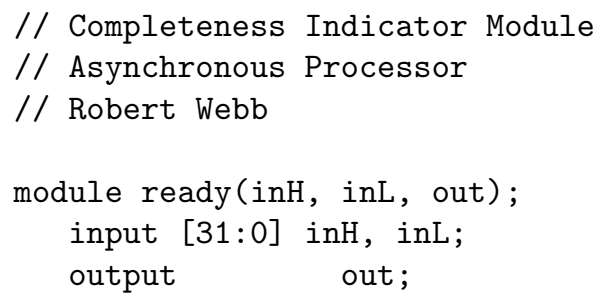

\footnotetext{
${ }^{2}$ It is important that the output of a flip-flop never be used to generate a completion signal in the way described because a flip-flop could enter a metastable state. It is not needed here to generate a completion signal from the register file here, so this detail can be omitted, but in the case a completion signal is needed it can be produced by simply delaying the request signal going into the register file by an upper bound of latency of the flip-flops.
} 


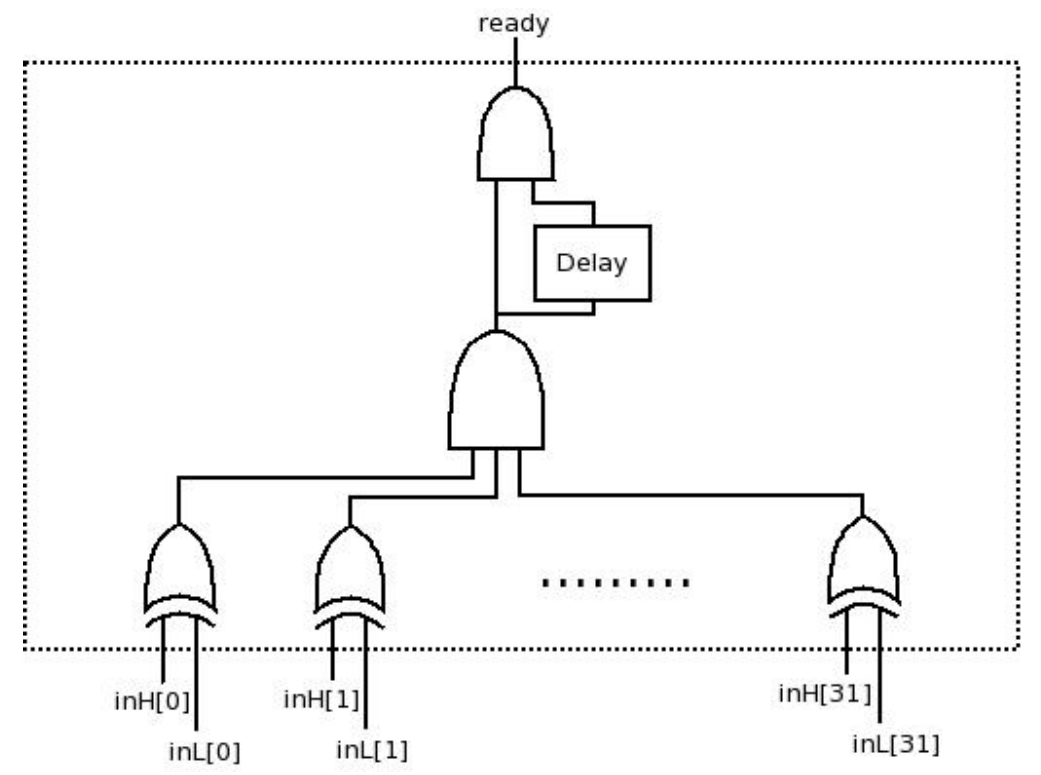

Figure 3.4: Completion Detector

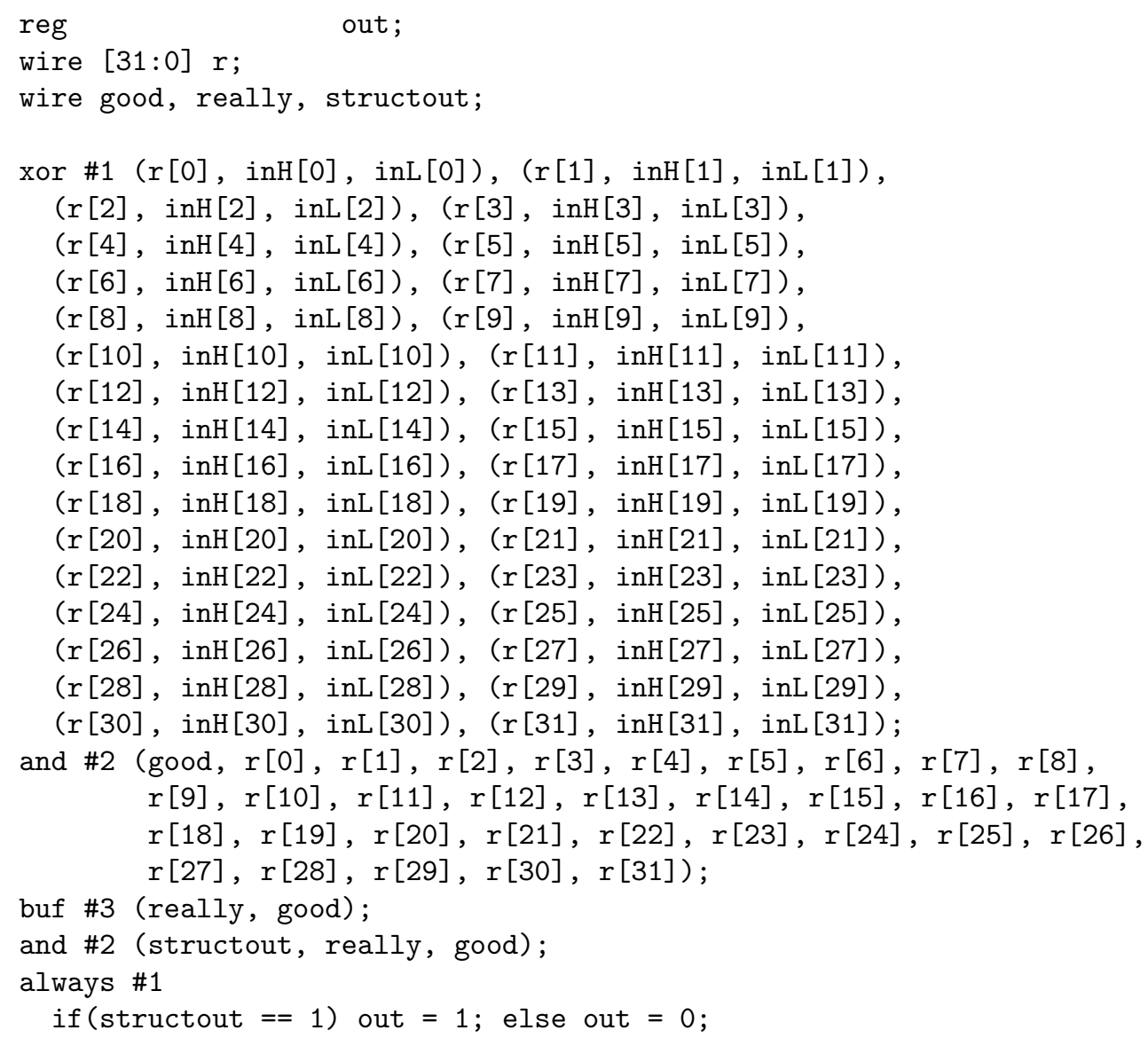




\subsubsection{Clock Removal}

In a single cycle implementation, the clock triggers 4 events:

1. Signal an update of the PC.

2. Signal a new instruction fetch.

3. Signal a write back of data to the register file.

4. Signal a write or read of the memory file.

The goal of the simulation is to somehow generate a "global completion signal" (GCS) which will replace the clock.

First, a distinction between the write or read function of the memory module is required. A read can happen when the ALU is finished because it is calculating the address of the read. This must occur before any GCS because the value of the read may be required by the register file, which will write back upon receiving the GCS. A write would occur upon receiving the GCS. Therefore, the memory module has been written to have distinct get and set signals. The get signal is connected to the ALUready signal. The set signal is connected to the GCS.

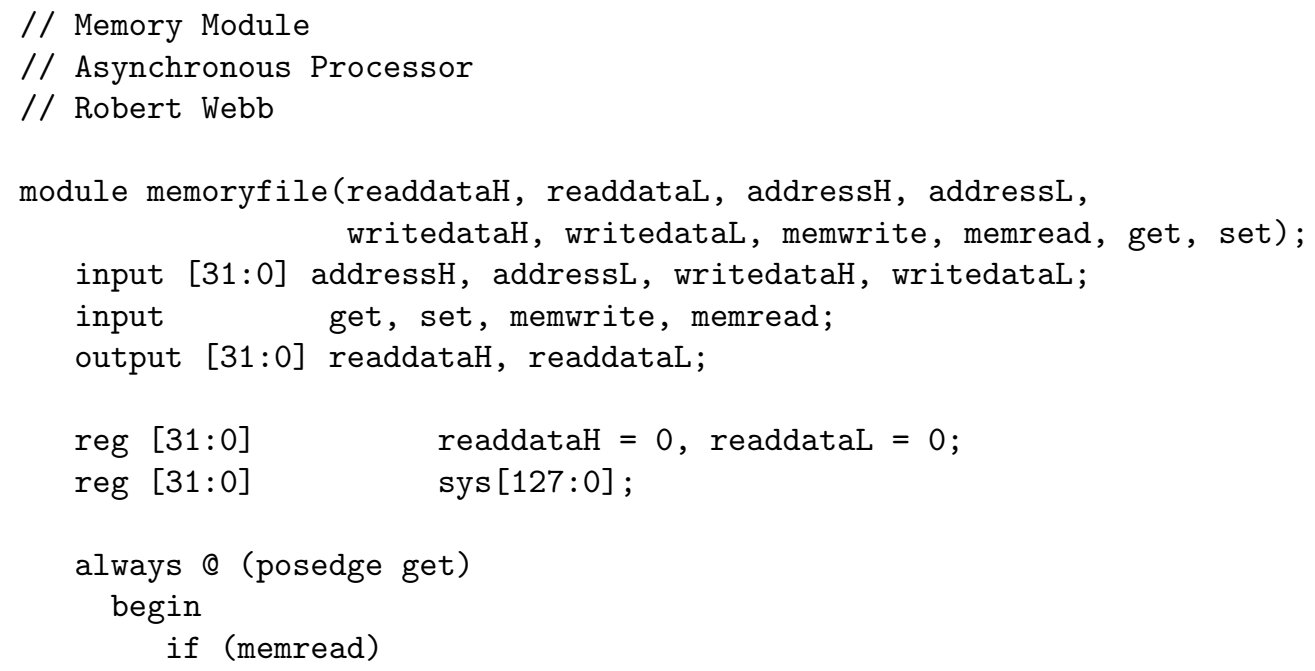




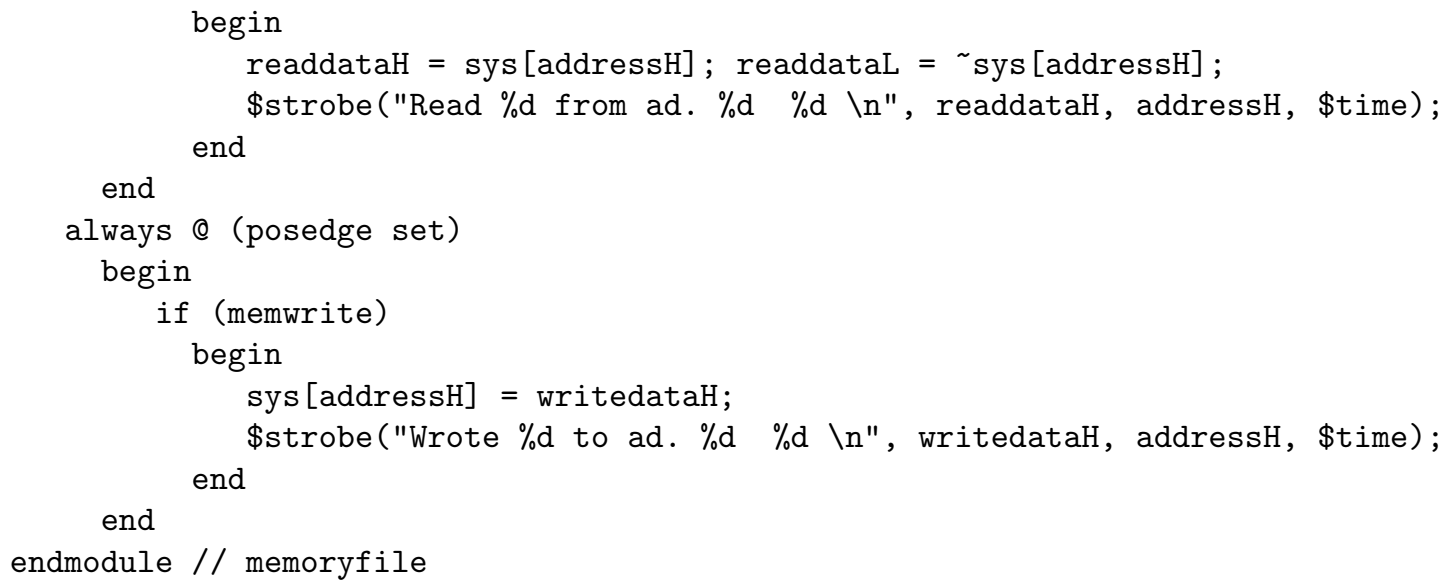

\subsubsection{Distribution of the GCS}

To fully remove the clock, three ready modules for the PC, the main ALU, and the final output are used. These ready signals are combined with an AND gate to produce the GCS. The global ready signal is sent back into the PC, instruction fetch, register, and memory modules. Appropriate delays for the GCS going into each module are required to ensure correct operation. Specifically, the ready signal must first perform any writes to the memory or register units before the state changes. The latch holding the current PC can then be updated. The instruction fetch module must wait for a stable output from the latch to lookup the next instruction. A high level schematic of the processor, without control signals, is shown in figure 3.5. The processor is now asynchronous and independent of a clock signal.

\subsection{Results and Discussion}

\subsubsection{Design Lessons}

When this design was complete, one major problem was found. Branch instructions always caused the processor to go to a garbage address. The problem 


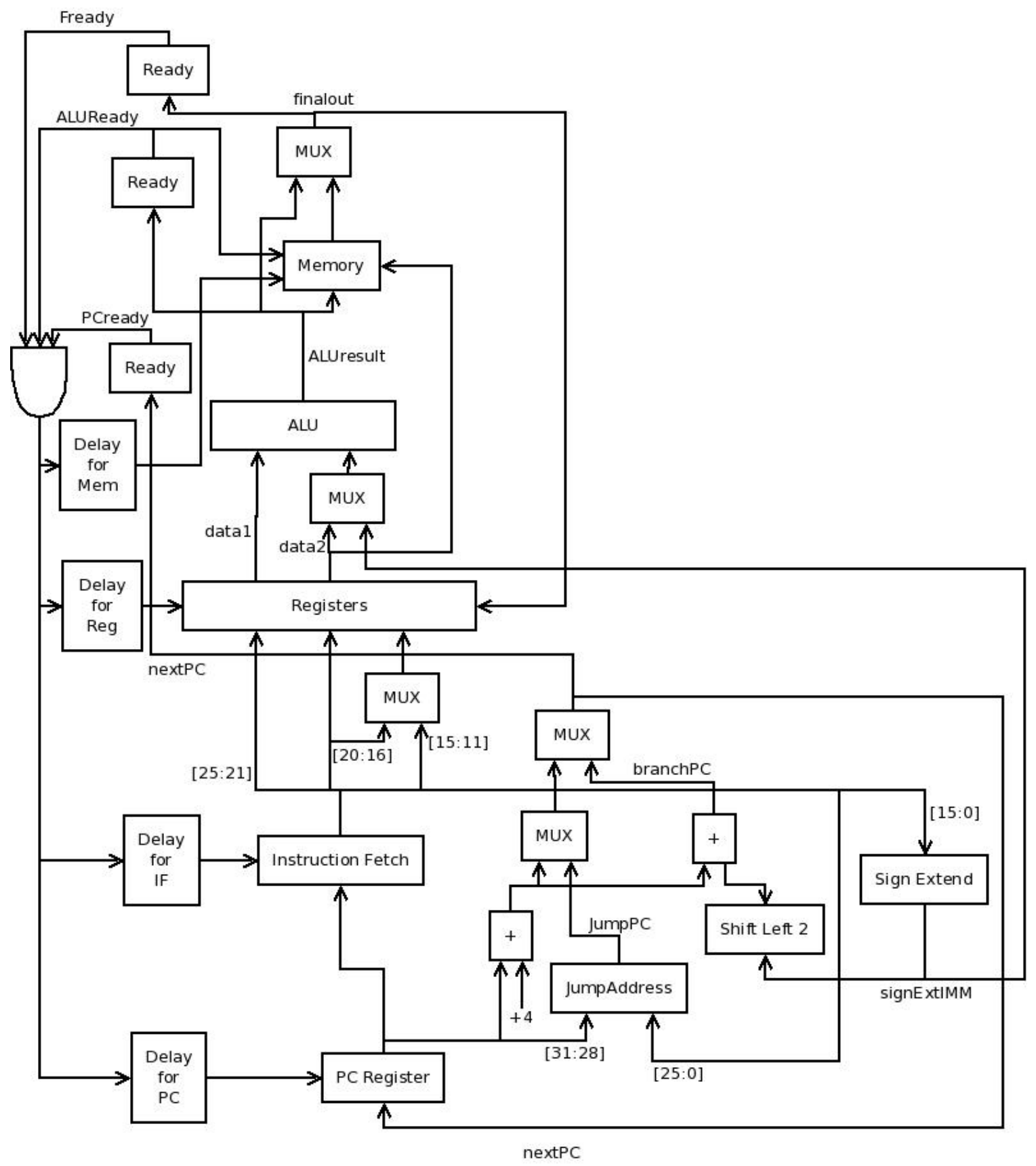

Figure 3.5: The single cycle asynchronous processor excluding control 
was the original design simply checked if the main ALU was finished before signaling the next state change. After tracing through the simulation several times, it was determined that the branch address ALU calculation was not completed, so the simulation was jumping to a garbage address. Adding completion signals to all the ALUs fixed the problem, which occurs because it takes much longer for a cascade adder to subtract a small number than to add a small number, and the branch instruction is performing a subtraction to calculate branchPC. The bug is instructive of the design issues that asynchronous processor designers must grapple with that may not be as pervasive in a synchronous environment.

\subsubsection{Results}

The same program that took the synchronous processor 13488 time units to complete was completed by the asynchronous version in 7604 time units. This is a $43.6 \%$ increase in computing speed.

A significant amount of the gain is from the simple arithmetic done at the beginning of the Fibonacci calculation. Simple arithmetic strongly favors the asynchronous implementation because it can be completed by the ALU quickly. One would expect the speed gains to be less significant with more complex calculations that approached the worst case delay of a synchronous design. 


\section{Chapter 4}

\section{An Asynchronous Pipelined}

\section{MIPS Processor}

\subsection{Theory}

As stated in chapter 2 the theory of asynchronous circuits begins with the "Speed Independent" (SI) model developed in the 50's and 60's by David Muller.[10] This theory assumes that all wires are ideal and have no delays and that gates can have arbitrary and potentially variable delay. A correctly designed SI circuit will never "glitch", meaning that once the inputs are stable, the output bits will make a single transition to the correct output. An example of a "glitch" is described in section 3.1.2. Theoretically correct asynchronous circuits will either produce a valid correct output, or an output that clearly communicates that it is not ready.

\subsubsection{Delay Insensitivity}

Early computers did not run at a speed where wire delay would be an issue. As silicon design began to increase both clock speed and the number of gates that 
could be used, wire delay became a significant part of the delay of the circuit. Because of this development, the logical next step was to develop a theory where wires and gates could have an arbitrary and potentially variable positive delay, known as DI. However, as was shown by Martin in [7], the class of circuits that are DI is very limited, so this theory was largely abandoned. When modern literature refers to DI, generally the assumptions are that of QDI.

\subsubsection{Quasi-Delay Insensitivity}

The standard compromise to DI is "Quasi-Delay Insensitivity" (QDI). Using the QDI paradigm a designer can assume that a wire that forks will have the same delay in a local area. These are called "iso-chronic forks". In other words, if a wire forks and goes to multiple nearby gates, a transition on that wire will reach the gates at the same time. It has been shown that these circuits are Turing complete.[2]

From an electrical engineering perspective, QDI is a reasonable assumption that can be guaranteed with careful layout of a processor. However, complexity to the design process in increased because the forks that need to be iso-chronic have to be identified and monitored through fabrication to ensure correct operation. In the simulation, the forks required to be iso-chronic are noted.

\subsubsection{The Muller C Element}

The basic building block of SI and QDI circuits is the Muller C element, first used in the ALU of Iliniac II. As noted in [12], the necessity of a new gate for asynchronous processors can be best described by the concept of "indication". A gate is said to indicate an input if the value of the input can be completely determined by the output of the gate. Consider the two input AND gate. A transition of the output to high indicates that both inputs are high. However, a transition to low indicates that only one input is low, so no information can be determined about one of the inputs. Transitioning to high indicates the state of 
both inputs, but transitioning to low indicates the state of only one. Similarly the OR gate also does not indicate both inputs on both transitions.

For correct SI or QDI behavior, a gate that indicates both inputs on both transitions is required. It is useful to consider joining two request signals. If both are high, then the output should be high, and if both are low, then the output should be low. If only one is high, then the output should be low. A high joined signal means that that the processing for both is finished. A low combined signal means that both units are ready to do something else. ${ }^{1}$ Therefore, if two requests are high and then one goes low, then their joined request should stay high because both of them are not ready to start processing again. The joined request should only go low when both of them are again low. This is the gate known as the Muller $\mathrm{C}$ element. It stays low until all inputs are high, then stays high until all inputs are low. The truth table is:

\begin{tabular}{c|c||c}
$\mathrm{A}$ & $\mathrm{B}$ & $\mathrm{C}$ \\
\hline 0 & 0 & 0 \\
1 & 0 & $\mathrm{C}_{t-1}$ \\
0 & 1 & $\mathrm{C}_{t-1}$ \\
1 & 1 & 1
\end{tabular}

The gate description and transistor implementation are shown in figure 4.1. The code that has been written for 2, 3, and 32 input $\mathrm{C}$ elements follows this paragraph. Behavioral Verilog is used because these units are considered atomic. While they could be expressed using AND and OR gates, defining the C element with real AND and OR gates could introduce "glitches" because of different delays in AND and OR gates.

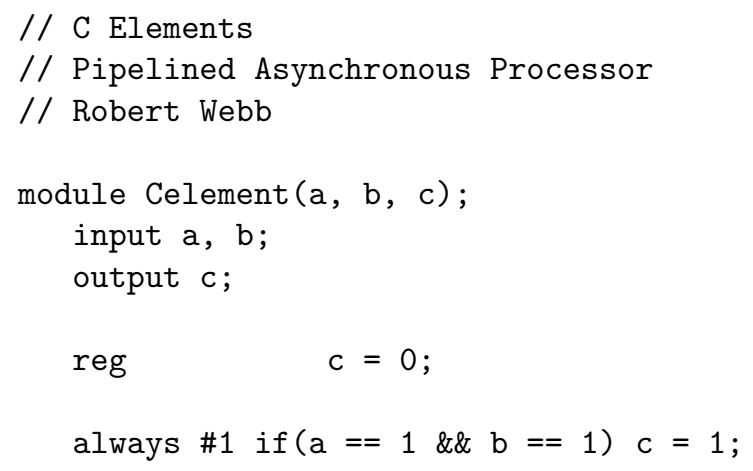

${ }^{1}$ Request signals will be more completely discussed in section 4.1.6. 


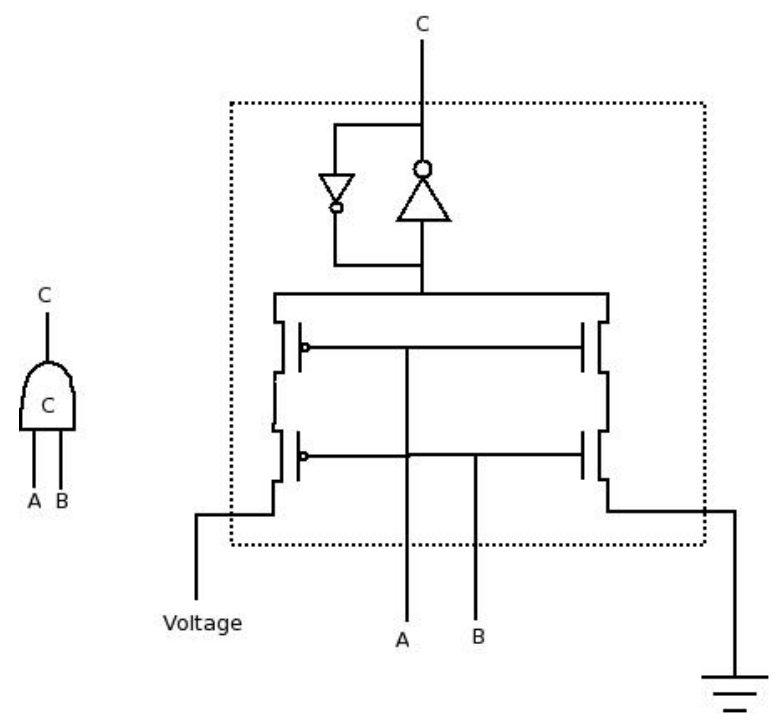

Figure 4.1: C element: gate description and transistor implementation

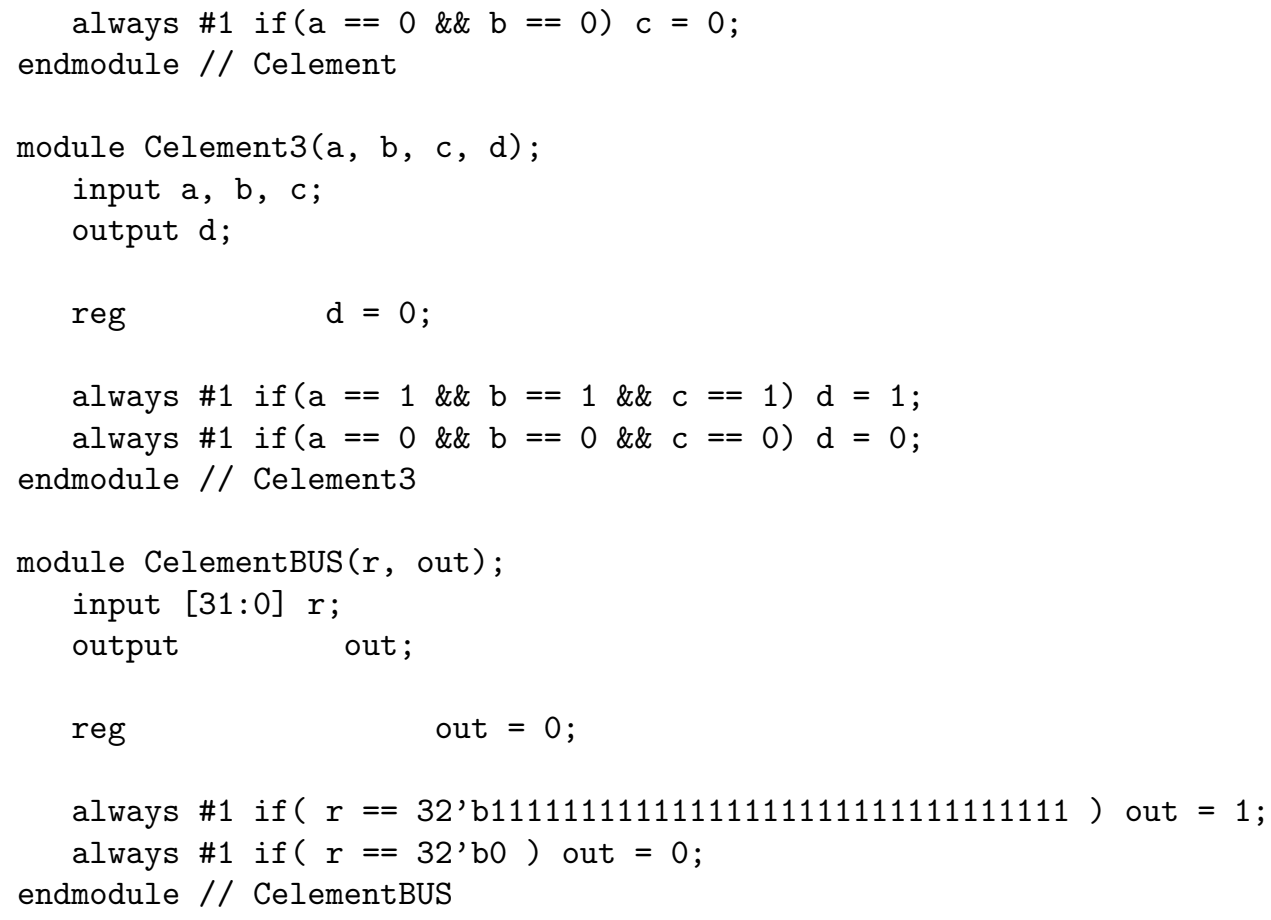

It should be noted that a $\mathrm{C}$ element has hysteresis, so is a form of latch. In fact, many asynchronous circuits use $\mathrm{C}$ elements as part of their state memory. An example is shown in the dual-rail pipeline registers in figures 4.11 and 4.12 . 


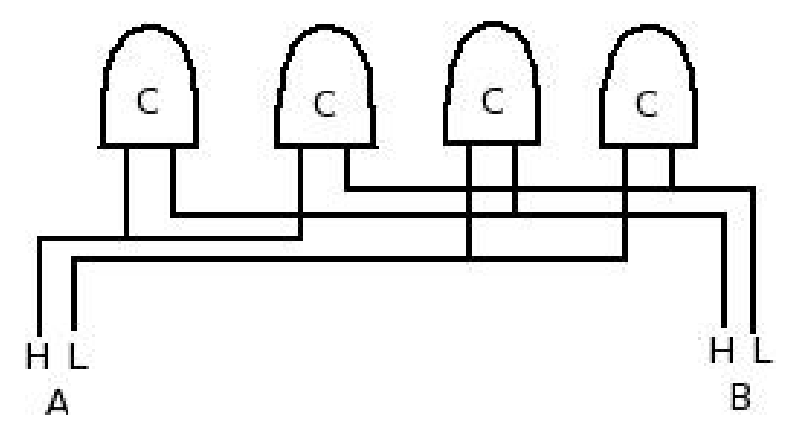

\section{Figure 4.2: C Element minterms for a 2 input DIMS circuit}

\subsubsection{DIMS}

Once $\mathrm{C}$ elements have been defined it is possible to make circuits that, by definition, cannot "glitch" using a systematic algorithm. The algorithm is called "Delay Insensitive Minterm Synthesis" or DIMS. Using this method all of the data must be in dual-rail format as defined in section 3.1. The value of $(1,1)$ is not allowed in this case. The circuits will be designed so that that combination will never be produced as long as that combination is never an input and all transitions on inputs are to or from $(0,0)$. Specifically, an input of $(1,0)$ can never transition directly to $(0,1)$ or vise-versa.

As an example, a simple 2 input circuit will be converted. ${ }^{2}$ Because there are 4 possible input states, there will be $4 \mathrm{C}$ elements in the circuit representing each possible minterm. ${ }^{3}$ Figure 4.2 represents the first stage of the DIMS circuit.

The truth table for the AND gate is:

\begin{tabular}{c|c||c}
$\mathrm{A}$ & $\mathrm{B}$ & $\mathrm{C}$ \\
\hline 0 & 0 & 0 \\
1 & 0 & 0 \\
0 & 1 & 0 \\
1 & 1 & 1
\end{tabular}

To complete the DIMS algorithm the $\mathrm{C}$ element outputs associated with each minterm are connected to the appropriate output. If there are multiple minterms for a given output, they can be connected with an OR gate as each minterm is

\footnotetext{
${ }^{2}$ Note that because these are dual-rail circuits, a 2 input circuit will have 4 input wires.

${ }^{3}$ If not all inputs are possible, then some of the $\mathrm{C}$ elements can be removed.
} 


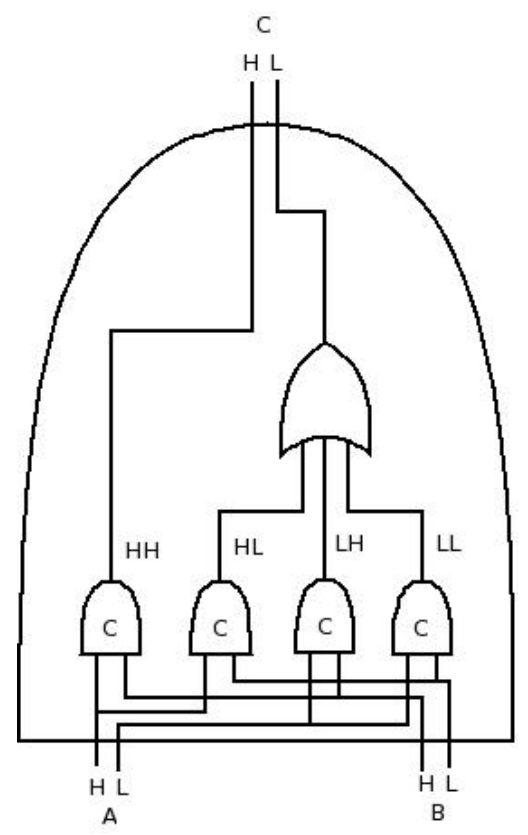

Figure 4.3: DIMS AND gate

exclusive from all the others. The resulting DIMS AND gate is show in figure 4.3. Using this method, it is simple to make a DIMS OR gate, as seen in figure 4.4. The verilog code for these modules also follows.

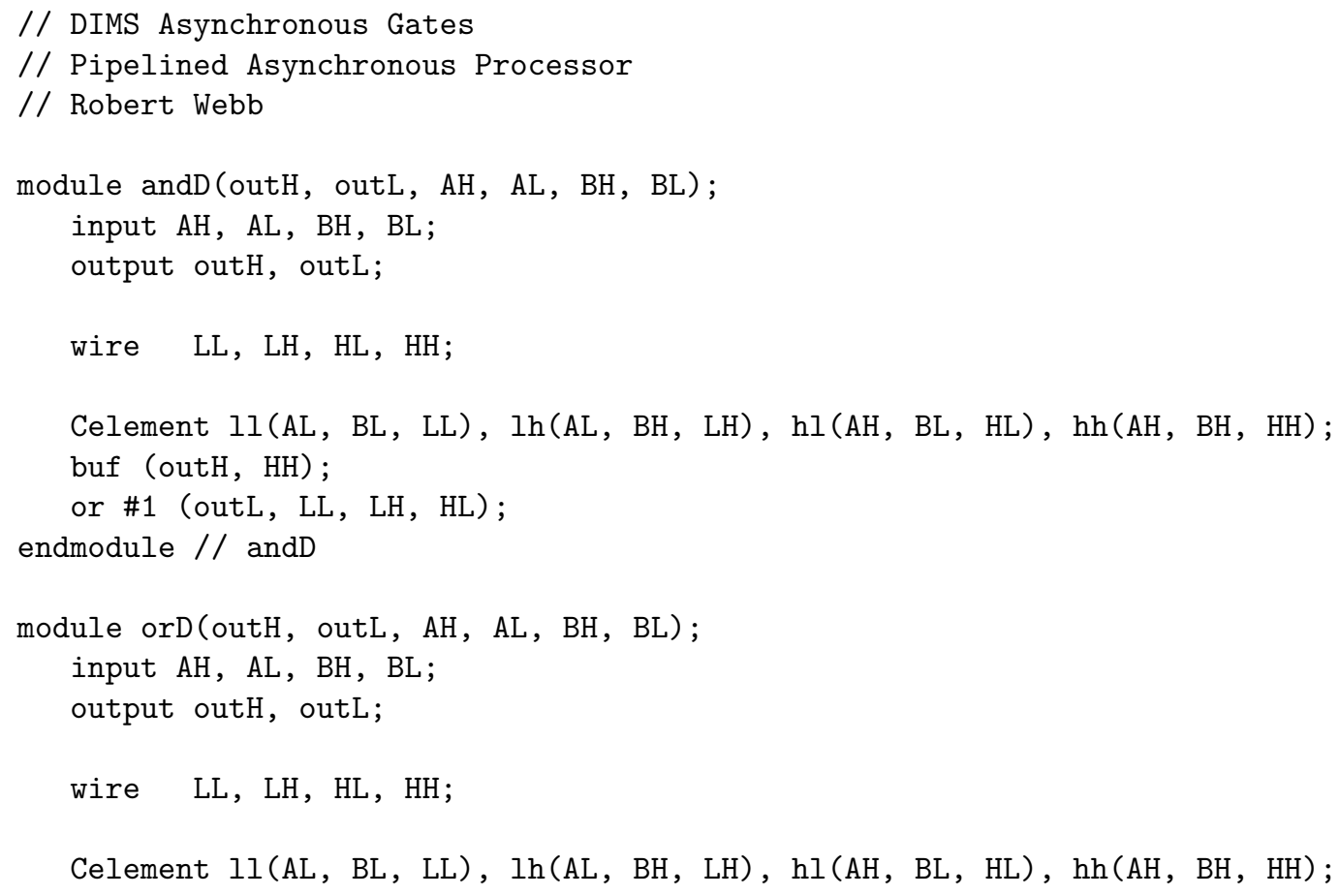




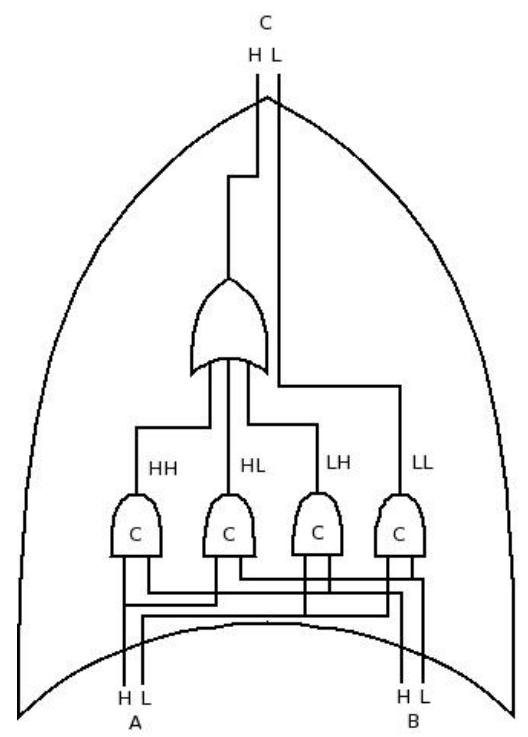

Figure 4.4: DIMS OR gate

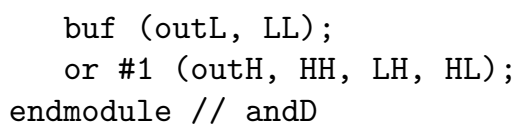

The resulting circuits will satisfy all the requirements of the SI theory and the QDI theory. The only delay assumption is that the forks of the wires going into the $\mathrm{C}$ elements are isochronic. However, to use these circuits, the inputs must begin in the undefined state (i.e. all bits are at $(0,0))$. By definition, in this state, the output will also be in the undefined state. The inputs can then change monotonically to their input state. In complex circuits with multiple outputs, the behavior of the outputs can be defined to be strongly indicating or weakly indicating. Strongly indicating circuits require all inputs to be defined before producing any output. Weakly indicating circuit outputs are allowed to become defined at different times and will produce output before all inputs are defined, if possible. For example, in the adder that is described in section 4.2 .2 , the single bit adders can produce output if the carry input is undefined because the carry out can be determined in some cases without the carry in value.

Once the output is defined, care must be taken to be sure that all inputs return to the undefined state and that the inputs remain undefined until the 
output has again returned to the undefined state. This is very important because of the hysteresis of the $\mathrm{C}$ elements. One possible consequence of not returning to the undefined state would be an output where both the high bit and low bit of an output is 1, which is not allowed in the circuit specification. The pipeline structure will ensure that the inputs and outputs return to the undefined state because the pipeline timing signals will be used to return the input to the undefined state and ensure the output has returned to the undefined state.

\subsubsection{Design Choices}

Clearly the new AND and OR gates are much more complicated. A traditional AND gate has 6 transistors and the new one has 30 transistors. For this reason, the approach of making new AND and OR modules and changing all of the gates in a synchronous processor as was done in chapter 3 is unrealistic. Instead of doubling the transistor count as before, there would be a quintupling of the transistor count. To mitigate this, when converting a circuit the DIMS procedure is done on larger modules to reduce replication of overhead. However, this can quickly become daunting. Assuming there are no possible optimizations, an $n$ input DIMS gate will have $2^{n} \mathrm{C}$ elements each having $n$ inputs!

Modules have been created to convert between dual-rail data and a bundled data encoding. In dual-rail encoding of data, the validity of the data is encoded as part of the data. This works well when the data is being used by combinatorial logic and makes the calculation of a completion signal much easier, but is very cumbersome in other parts of a data path such as multiplexors and registers. For these parts of the data path bundled data is used. Bundled data is the traditional encoding of the data with an additional bit indicating if the data is valid. This additional bit is usually referred to as a "request" because if it is high, then the data is requesting to be consumed by some subsequent processing unit.

The dual-rail to bundled data converter is labeled in schematics as "SR", and the bundled data to dual -rail converter is labeled as "DR". The circuit diagram for the "DR" converter is in figure 4.5. The circuit diagram for the 


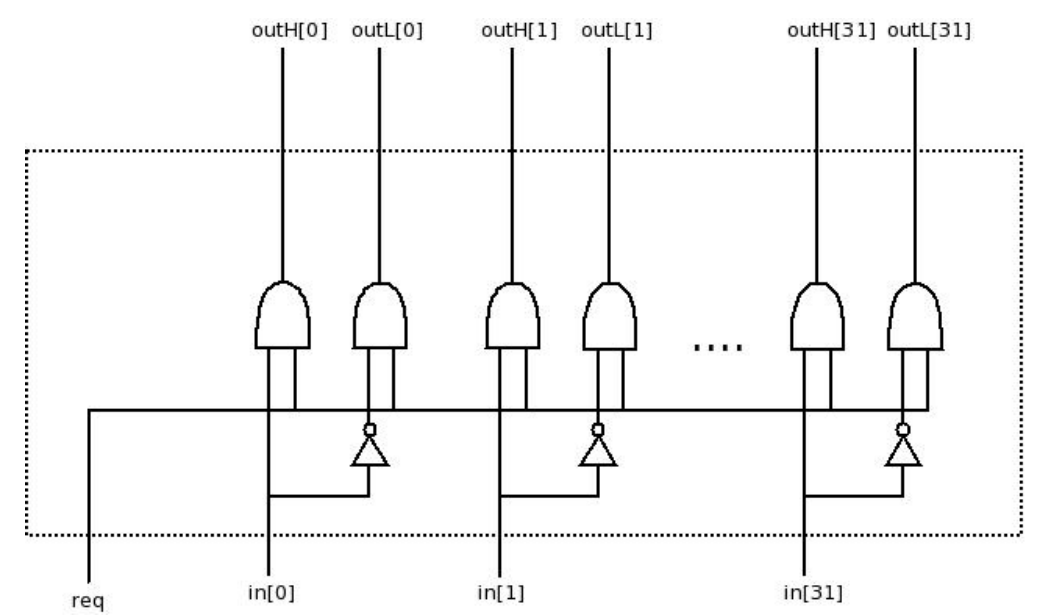

\section{Figure 4.5: Conversion Unit, Bundled Data into Dual-rail}

"SR" converter is in figure 4.6. The Verilog code for these modules also follows. The "DR" module is very important because it is how the input for a DIMS unit will return to the undefined state before transitioning to the next set of data inputs. The req input to the "DR" cannot go high until the req output of the "SR" goes low. Most of these control issues will be handled by the pipeline control units defined in section 4.1.6.

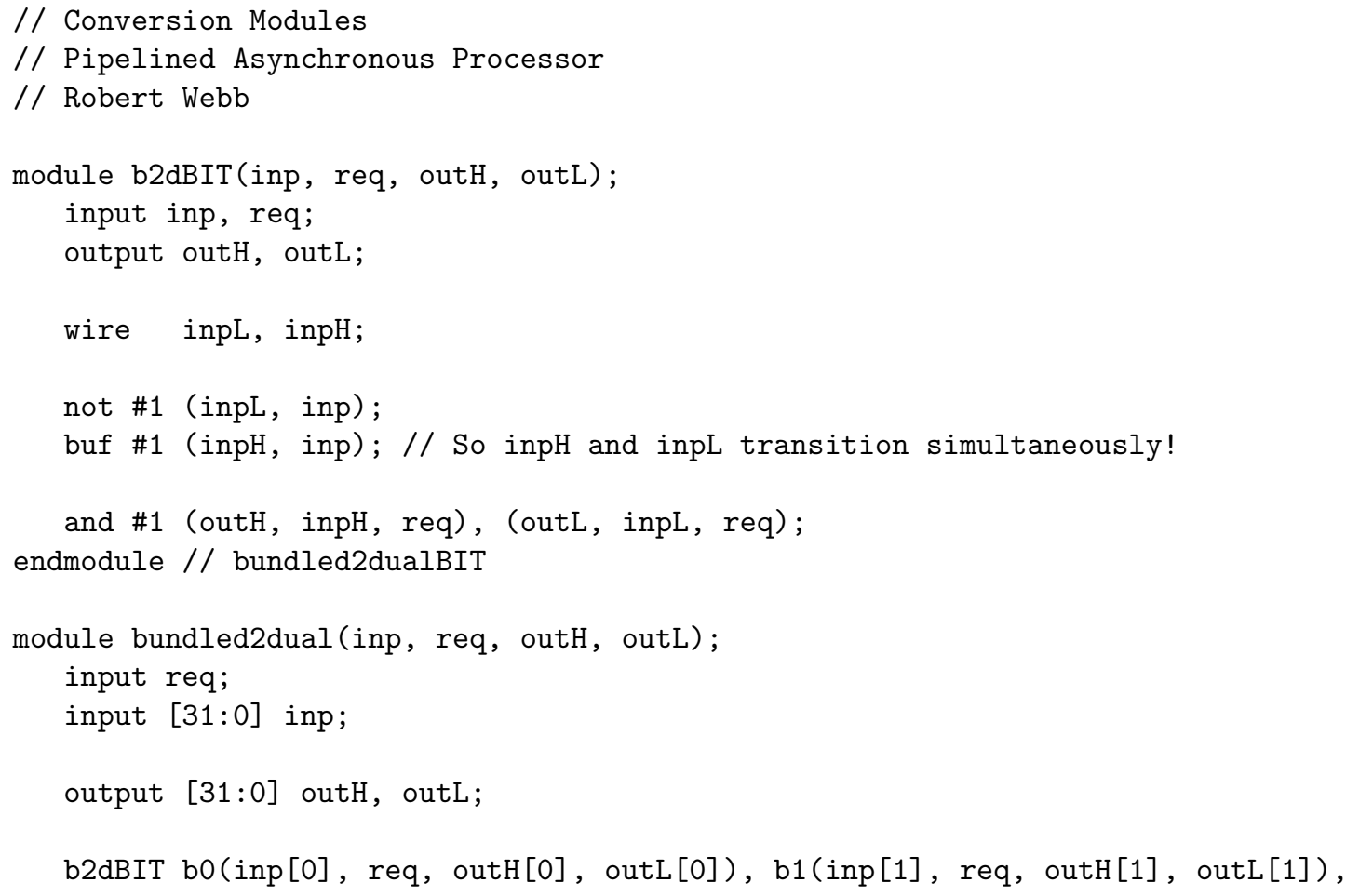




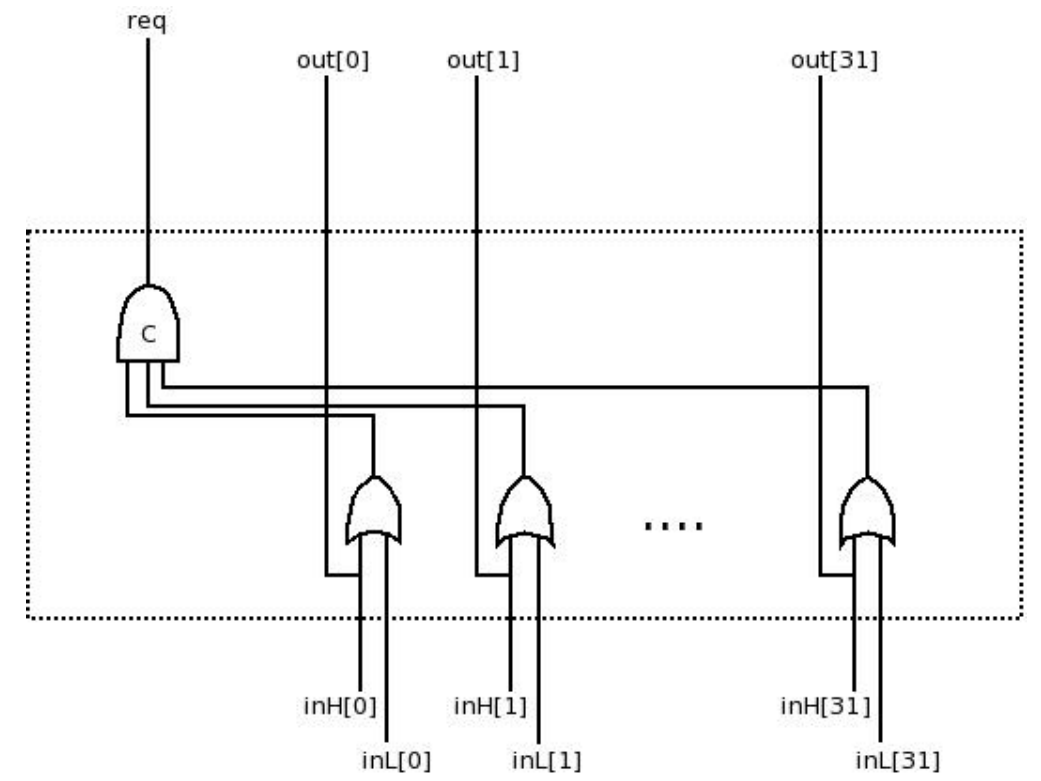

Figure 4.6: Conversion Unit, Dual-rail into Bundled Data

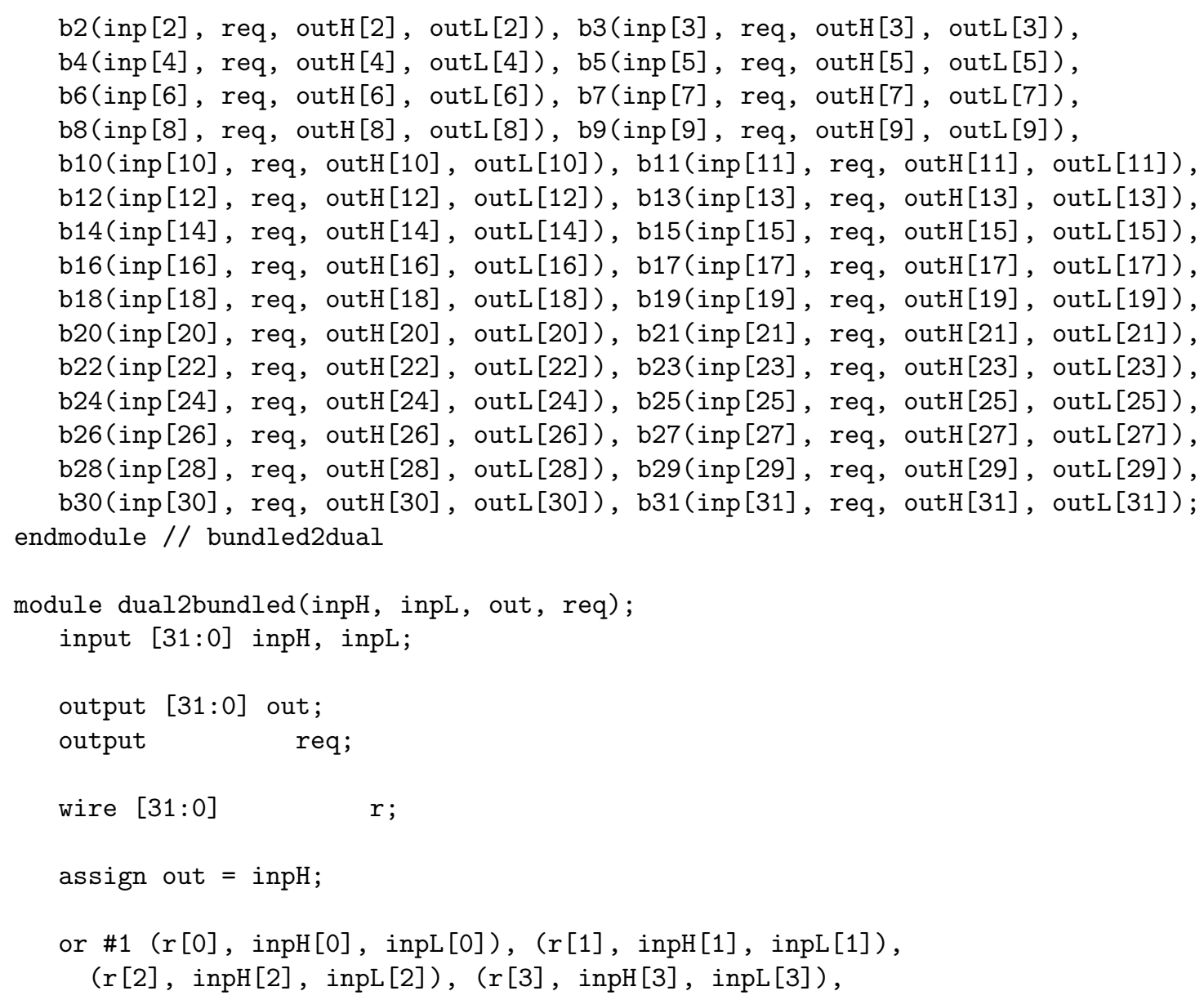




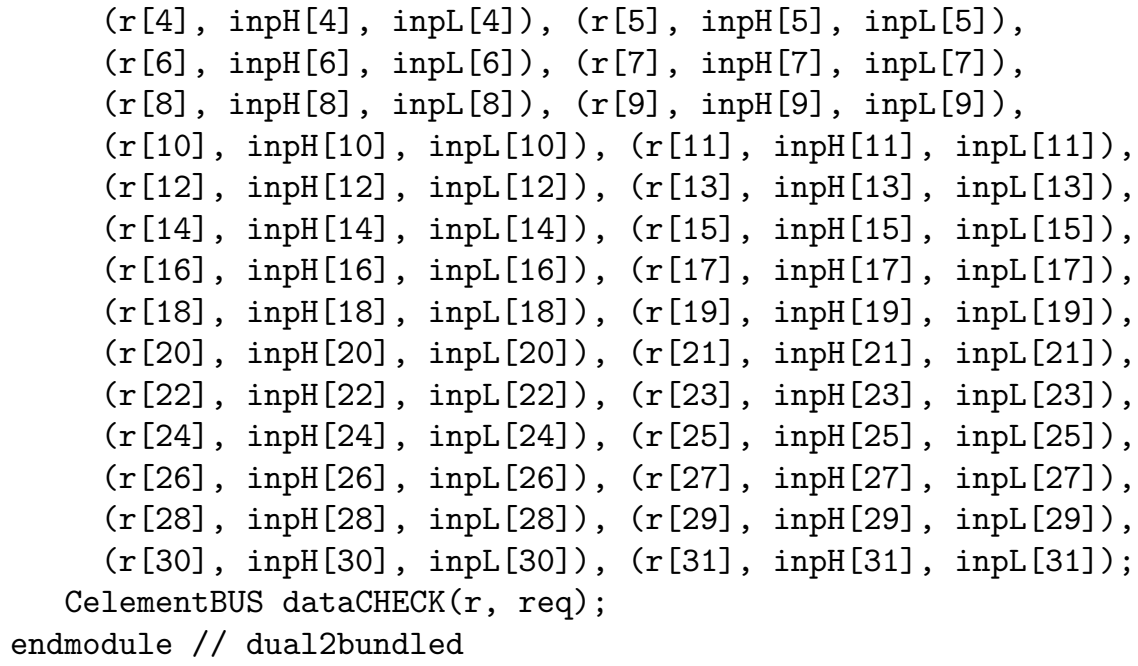

\subsubsection{Muller Pipeline}

To understand the asynchronous meaning of a pipeline, it is useful to think about a line of people passing buckets of water to a fire crew. If this line were a synchronous pipeline, everyone would be passing buckets at exactly the same frequency and buckets full of water would appear at the beginning of the pipeline at that exact frequency. However, in an asynchronous pipeline, everyone on the line would be working at their own pace, and each individual pace could change over time. If one person was slow, then everyone before that person in the line would have to wait to pass the next bucket. If a group of people at the end of the line were all fast, then when a bucket got to that part of the line it would speed through, and those people would be waiting idle for the next bucket.

What is required is a handshaking protocol in the form of requests and acknowledgments. The solution, called the four-phase handshake protocol and generally credited to Muller, focuses on the actions of an individual unit of the circuit.[12] Each unit has four timing signals, two requests for receiving and passing along and two acknowledges, for receiving and passing along. The wave forms of corresponding request and acknowledge signals are shown in figure 4.7.

A unit begins at an idle state where all timing signals are low. For simplicity, the unit will be referred to as current unit $(\mathrm{CU})$. The previous unit (PU) and next 


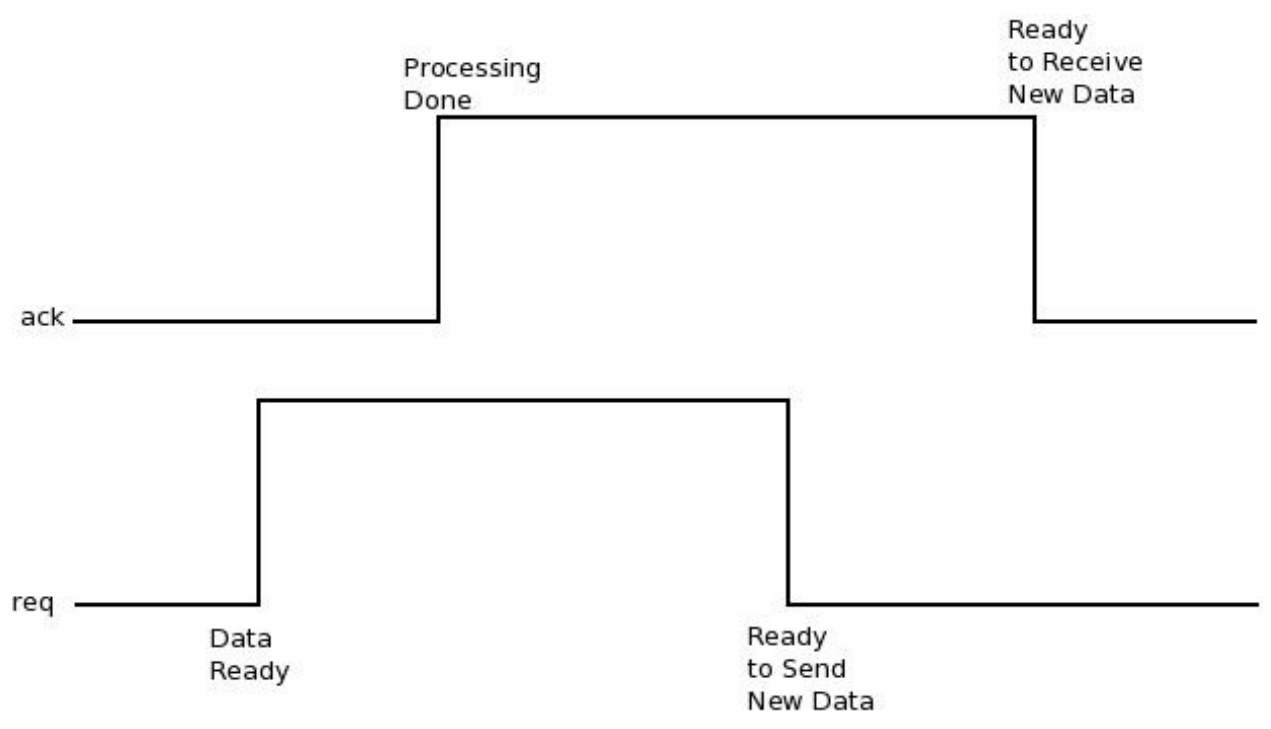

Figure 4.7: The 4 phase handshake protocol waveform

unit (NU) will also be referenced. All signals are currently low. At this point, $\mathrm{CU}$ is ready to receive a request from $\mathrm{PU}$. When the request from $\mathrm{PU}$ is received, $\mathrm{CU}$ does its work, then sends a request ahead to NU. CU now must wait. At this point, $\mathrm{CU}$ is still receiving a request from $\mathrm{PU}$, because no acknowledgement has been send back to PU. Also, CU has to continue to output the current work until $\mathrm{NU}$ acknowledges receipt. When $\mathrm{CU}$ gets acknowledgement from NU, CU can send an acknowledgement back to PU. When CU sends the acknowledge back, it is not to say "I have it", it is to say "I have successfully passed it on" ${ }^{4}$ When the acknowledge goes high, that does not communicate that NU is ready for new data. Recall back to section 4.1.4, once a DIMS circuit is finished, it must go back to the neutral state. CU can't go back to the neutral state right now, because the request from PU is still high and the acknowledge from NU is still high. However, PU will lower its request once it receives acknowledgement. When PU lowers its request, the inputs to $\mathrm{CU}$ will be in the neutral state. The neutral inputs will propagate through the logic in $\mathrm{CU}$, and when the outputs are also neutral, the request to NU will go low. Once NU has returned to the neutral state, it will set

\footnotetext{
${ }^{4}$ This is important because CU needs to have valid inputs, which are signaled by the request from the previous unit, and when $\mathrm{CU}$ produces output, that output must be put somewhere (usually some sort of pipeline register) before the inputs change.
} 


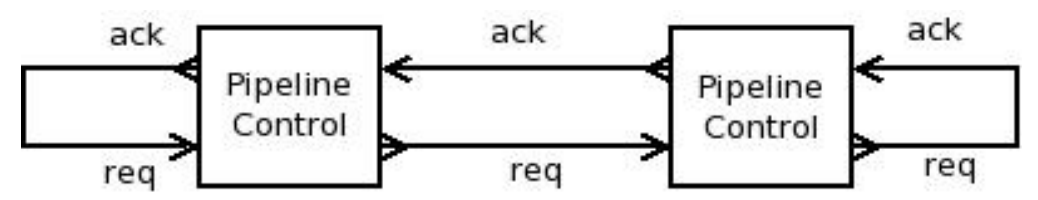

Figure 4.8: A basic asynchronous pipeline made from pipeline control units

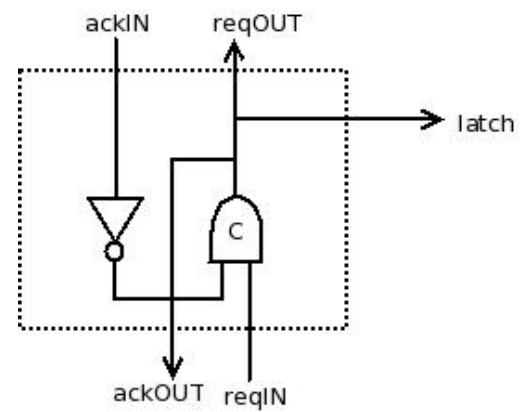

\section{Figure 4.9: Muller Pipeline Unit}

acknowledge low. $\mathrm{CU}$ can then set acknowledge to $\mathrm{PU}$ low. $\mathrm{CU}$ is now ready to get a new request, as all the signals have returned to 0 .

To simplify the details described above, a pipeline control unit is needed. This unit will deal with most of the handshaking independent of the actual processing units. The pipeline control unit can be copied and placed in sequence to coordinate the handshaking actions. The processing units would then only be required to receive a request and generate a request.

The use of this simple pipeline control unit is show in figure 4.8. Muller designed a circuit that would produce this behavior, as shown in figure 4.9. In addition to generating the request and acknowledge signals, this gate also generates a latch enable, so that data can be carried down the pipe with the signals as shown in figure 4.10. An alternative pipeline control unit can be used with dual-rail data as shown in figure 4.11. The $\mathrm{C}$ elements in this pipeline control unit are used instead of a traditional latch. This idea can be extended to multiple bits of dual-rail data as shown in 4.12.

As show in figure 4.10, all the pipeline does is propagate data down each of the stages. To make this useful processing units need to be added. For the 


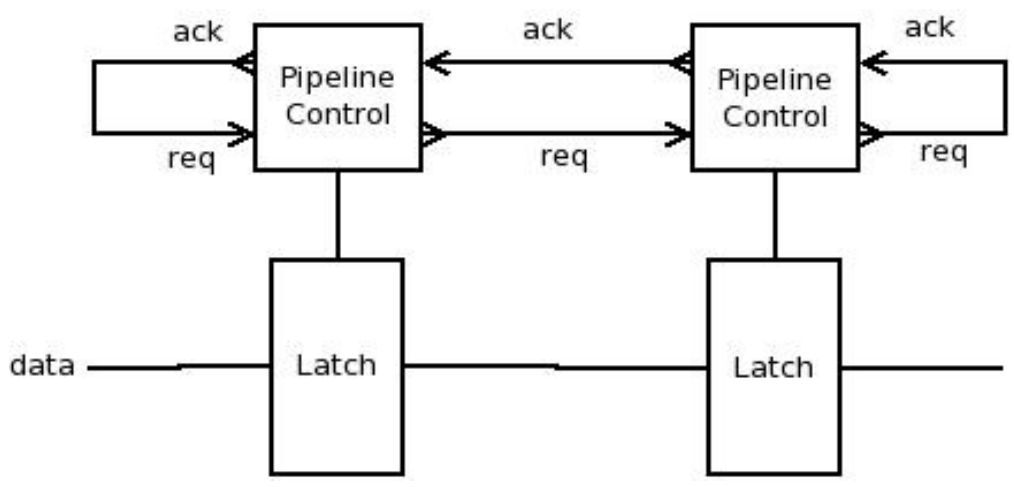

Figure 4.10: A basic asynchronous pipeline with latches

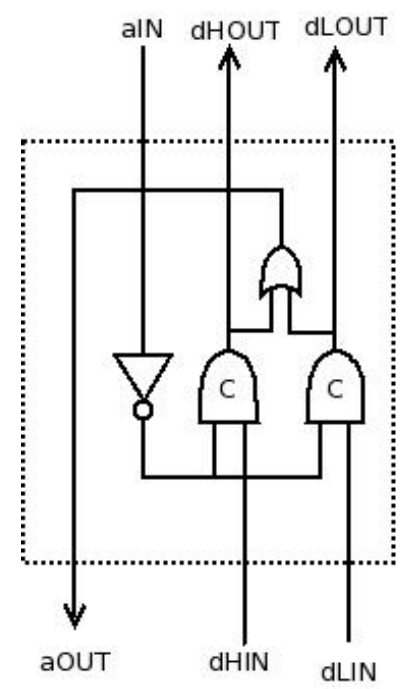

Figure 4.11: Dual-Rail Pipeline Unit 


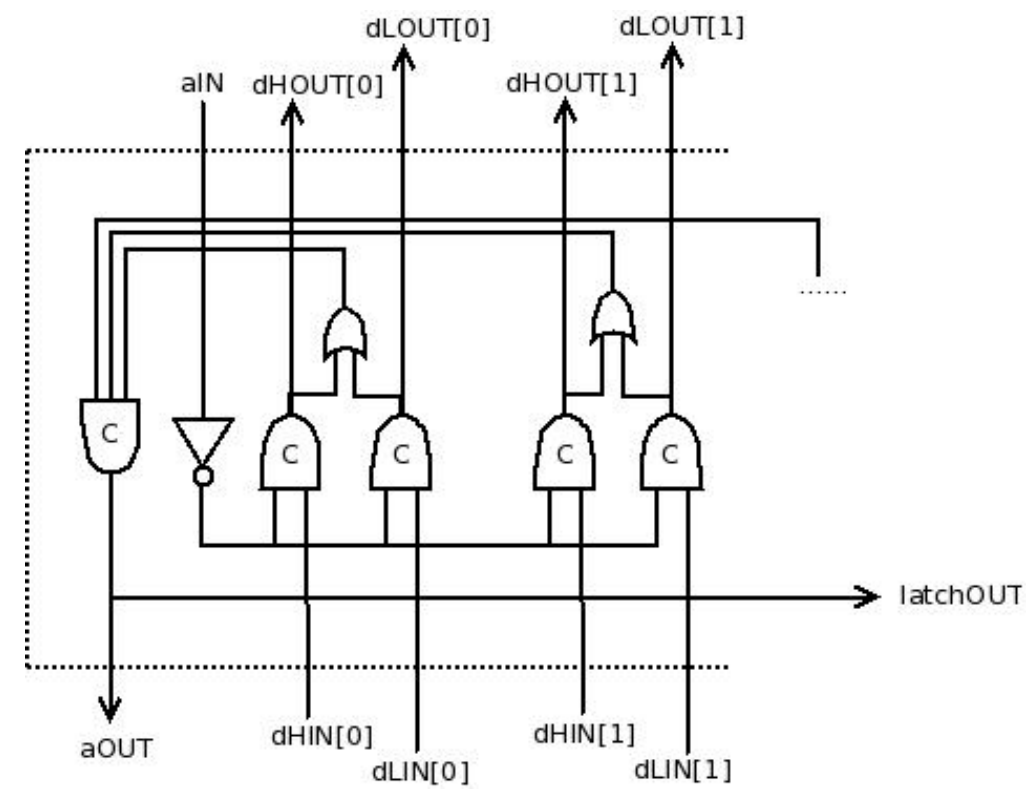

\section{Figure 4.12: Dual-Rail Pipeline Gate with Multiple data Inputs}

purposes of this paper, there are two kinds of processing units: constant delay units and variable delay units. Inserting a constant delay unit like a register file is shown in figure 4.13. The only variable delay unit in this project is the ALU. Inserting one of those is a bit more complex because the data is in bundled form when traveling down the pipeline, so the converters defined in figures 4.5 and 4.6 must be used. The result is shown in figure 4.14.

For the ALU to function correctly the inputs must return to the neutral state before the next set of values become defined. Initially all the values of the timing signals are 0 which causes the DR module to only output the neutral state. First reqIN $=1$ which allows the ALU to begin work by defining the inputs. Then reqOUT $=1$ when it is finished because the SR module generates a completion signal. Then ackIN $=1$ when the next phase has latched the data, and ackOUT $=1$ because it is directly connected to ackIN. Because of the acknowledge, reqIN $=0$ when the previous unit lowers its request and the ALU inputs go to neutral because of the DR unit. After processing, the ALU outputs go neutral and SR unit causes reqOUT $=0$. This allows ack $=0$, and the initial state has been restored. 


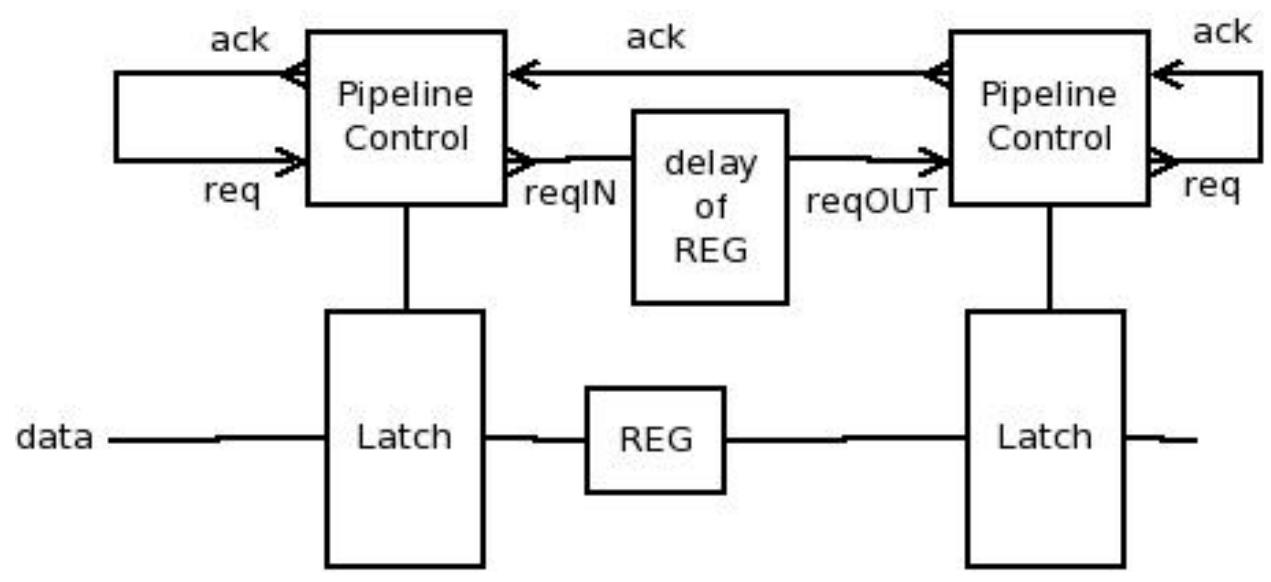

Figure 4.13: A basic asynchronous pipeline with a constant delay processing unit

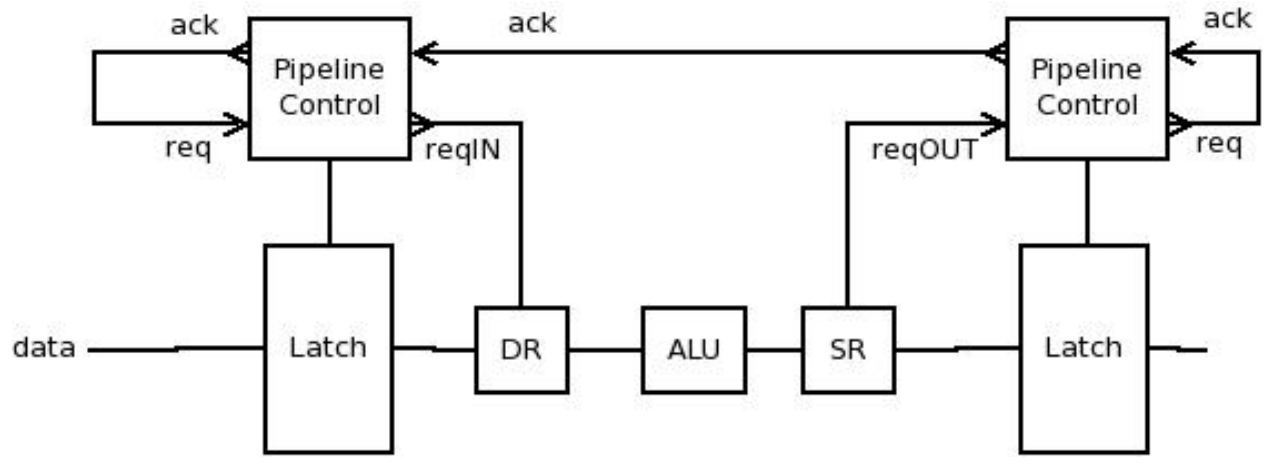

Figure 4.14: A basic asynchronous pipeline with a variable delay processing unit 


\subsection{Simulation}

With section 4.1 completed, the necessary theory to complete an asynchronous MIPS pipeline is available.

\subsubsection{Data Path Decomposition}

Asynchronous MIPS data paths are generally decomposed into 3 stages: Instruction Fetch, Instruction Decode, and Execute. The memory and write-back stages of a traditional five stage MIPS pipeline are incorporated into the execute stage because many of those operations can occur in parallel if some circuitry is duplicated. This parallel execution can be exploited to a great degree in asynchronous processors. For a more detailed discussion of this point, see [3, 8].

\section{Instruction Fetch}

The Instruction Fetch (IF) stage is pictured in figure 4.15. Because this is the first stage, it must generate the first request. This is done by combining the request from the two main units of this stage in a $\mathrm{C}$ element. The IF unit, because it is a memory unit, will generate its own request when memory reads have been completed. The code that defines the behavior of the IF unit is in appendix C. The adder that calculates the $\mathrm{PC}$ incremented by 4 will also generate a completion signal when it has finished its work. It should be noted that since there is no request coming into the instruction fetch stage, the validity of the inputs to the adder, which is a DIMS implementation, is determined by the opposite of the acknowledge from the Instruction Decode (ID) stage.

Jumps are handled entirely in this stage by simply cycling through instructions and the $\mathrm{PC}$ that is determined by the jump instructions until a non-jump instruction is found. This process is entirely transparent to the subsequent stages. It appears to them as though jump instructions are simply never encountered. This also means that a branch-delay-slot after a jump is unnecessary. 


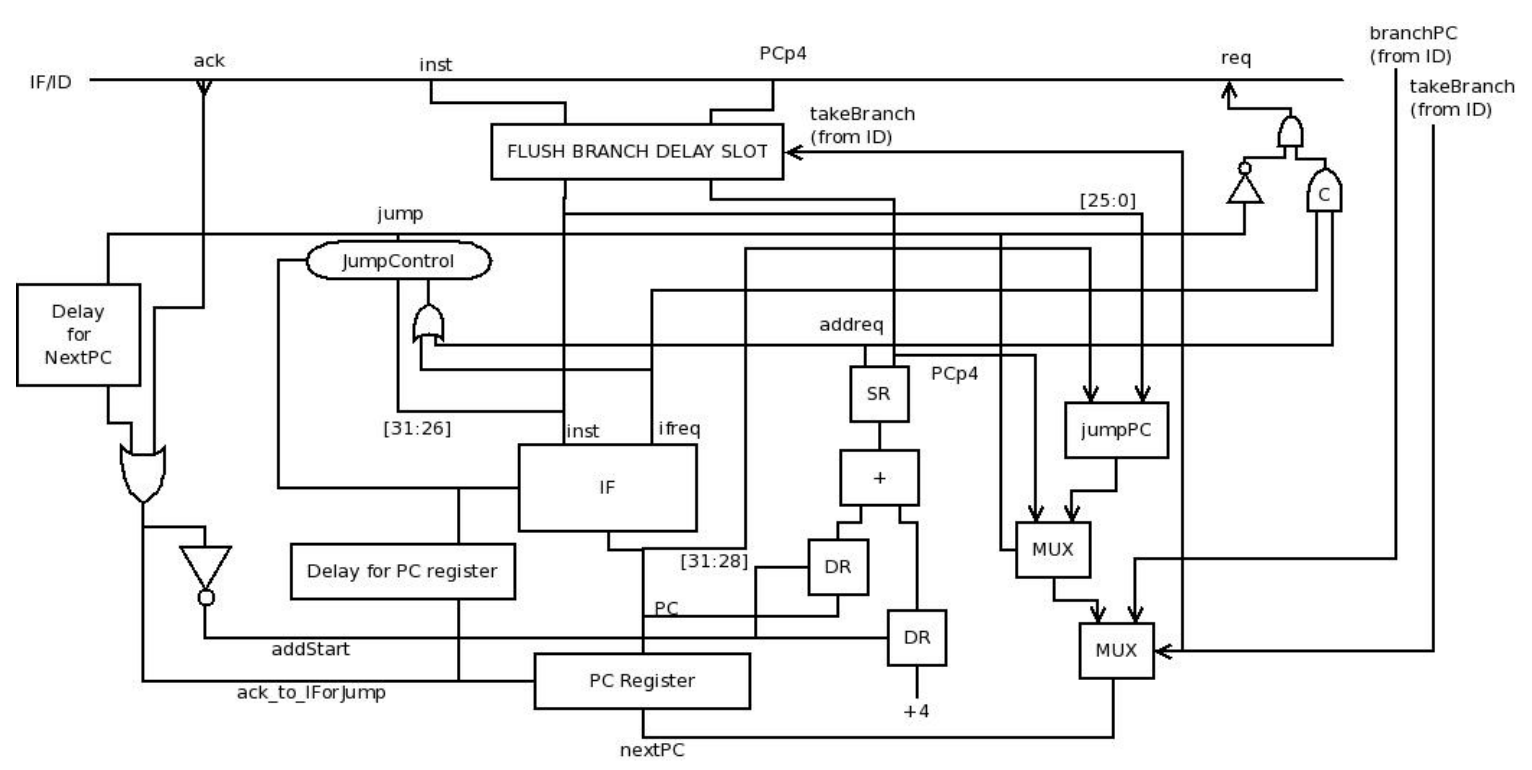

Figure 4.15: Instruction Fetch Stage with control for jump commands

The IF stage attempts to fetch and pass the instruction following a branch regardless of the branch outcome. However, because the IF stage cannot move forward without the acknowledgement of the ID stage, the branchPC and takeBranch flag will be valid before the nextPC is written into the PC register, and the takeBranch flag can flush the branch delay slot instruction before the values are saved by the pipeline registers.

\section{Instruction Decode}

The ID stage is pictured in figure 4.16. The control signals are generated, and the reads from the register file are completed. The request going forward to the Execute (EX) stage is generated by the constant delay of the multiplexors and latches in the register file and the request from the adder that is calculating the branchPC. As the adder is frequently adding a negative number, the delay for this branch becomes significant because all the bits generally have to wait for a long ripple carry. To deal with this problem, if the branchPC is not needed, the unit does not wait for the request from the adder.

The positive edge of acknowledge from EX is used to signal a write back into 


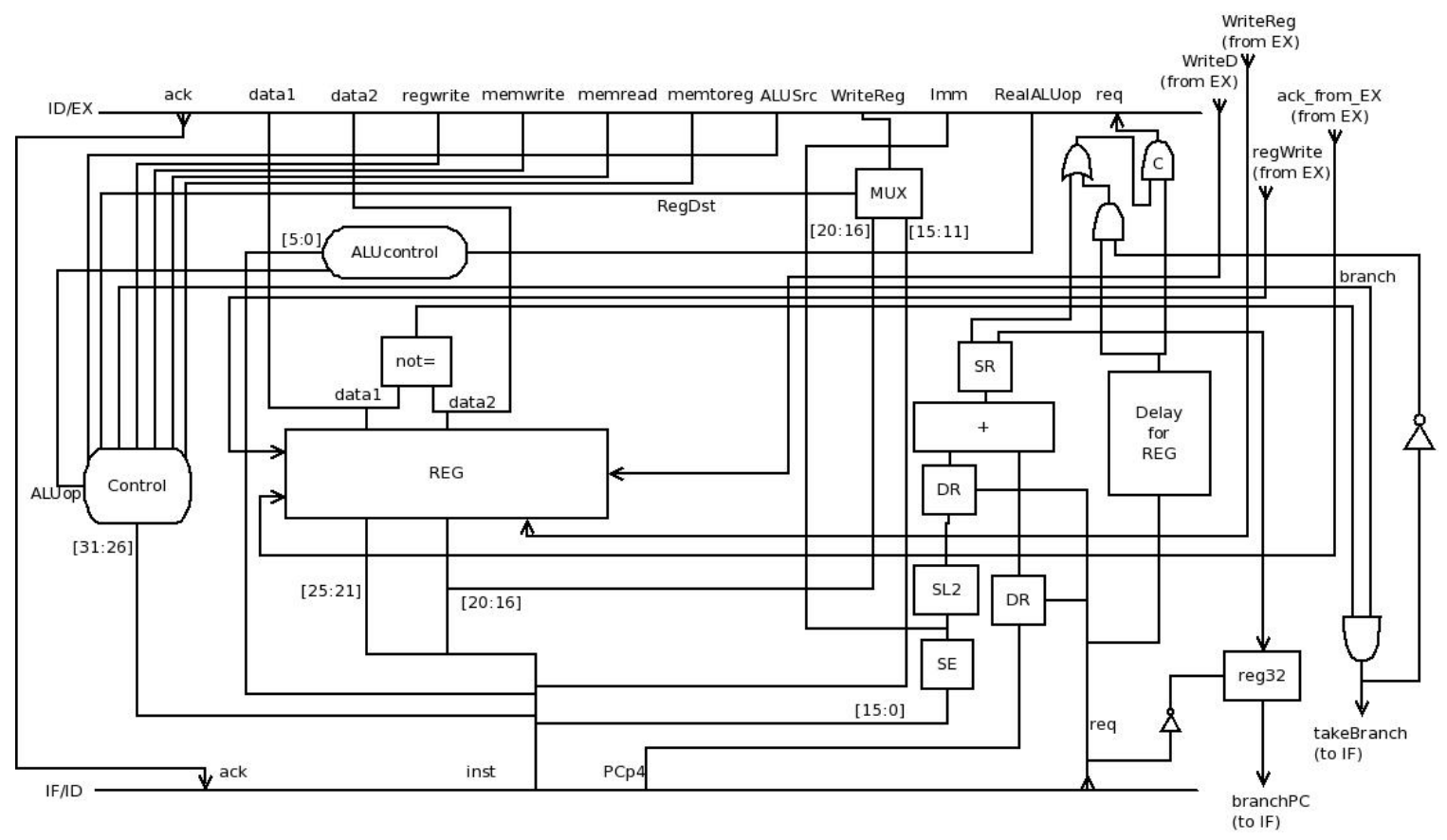

Figure 4.16: Instruction Decode Stage with control and branch decision

the register file. This means that there is no need for a forwarding unit as the pipeline register latches the data coming from the register file on the following negative edge of the acknowledge, which is more than enough time to ensure that the data has been written through.

A latch for the branchPC that is triggered by the negative edge of the request into ID is necessary because when the request goes low, the values going into the adder return to the neutral state and eventually branchPC also returns to the neutral state, which looks like a zero after it has gone through the conversion to bundled data unit. Because the branchPC is not used until the acknowledge to IF is set, its value needs to be saved. ${ }^{5}$

\section{Execute}

The EX stage is pictured in figure 4.17. There are a few things worth noting. First there is a delay on the request so the multiplexor that chooses the second

\footnotetext{
${ }^{5}$ This was a terrible bug to try to find. One doesn't usually think about outputs of an ALU going to 0 between every transition. Every branch was a jump to 0 for much to long...
} 
operand for the ALU is completed before the operands are converted into dualrail encoding. This is very important because the inputs must make a single change from neutral to their valid state. If the multiplexor is not stable the output of the ALU could be invalidated. The ALU produces a request which is passed through to the memory module. The memory is external and generates its own completion signal. The code defining the behavior of the memory module is in appendix C. In the case that memory is not used, the ALU request is simply passed through. In the case of a write the request is passed through and the write is done asynchronously. In the case of a read, then additional delay is produced while the read takes place. This delay could be highly variable because of the different levels of memory cache. The signal is then delayed because the multiplexor that chooses the write back value must be completed before the acknowledge is given.

Because of the intended audience of the simulation, there is not much happening in this stage. However, most of the performance improvement can come from here. In the Cal Tech designs this stage was more of a macro stage with many finely pipelined and mostly concurrent processing units. For example, the memory module had its own adder for address calculation. This allowed a read instruction to be dispatched to the memory module, and if there was a cache miss and long delay, the IF and ID stages could continue to dispatch independent instructions to several executions units in parallel. Because there were multiple execution units, there were several busses to write back to the register file. A forwarding unit was required in this case because the writes to the register file happened asynchronously and there was no guarantee that a write would happen before it was needed for the an instruction being dispatched to a different execution unit. Even given all the complexity, the forwarding unit is still more simple than the one required for the 5 stage asynchronous MIPS pipeline. 


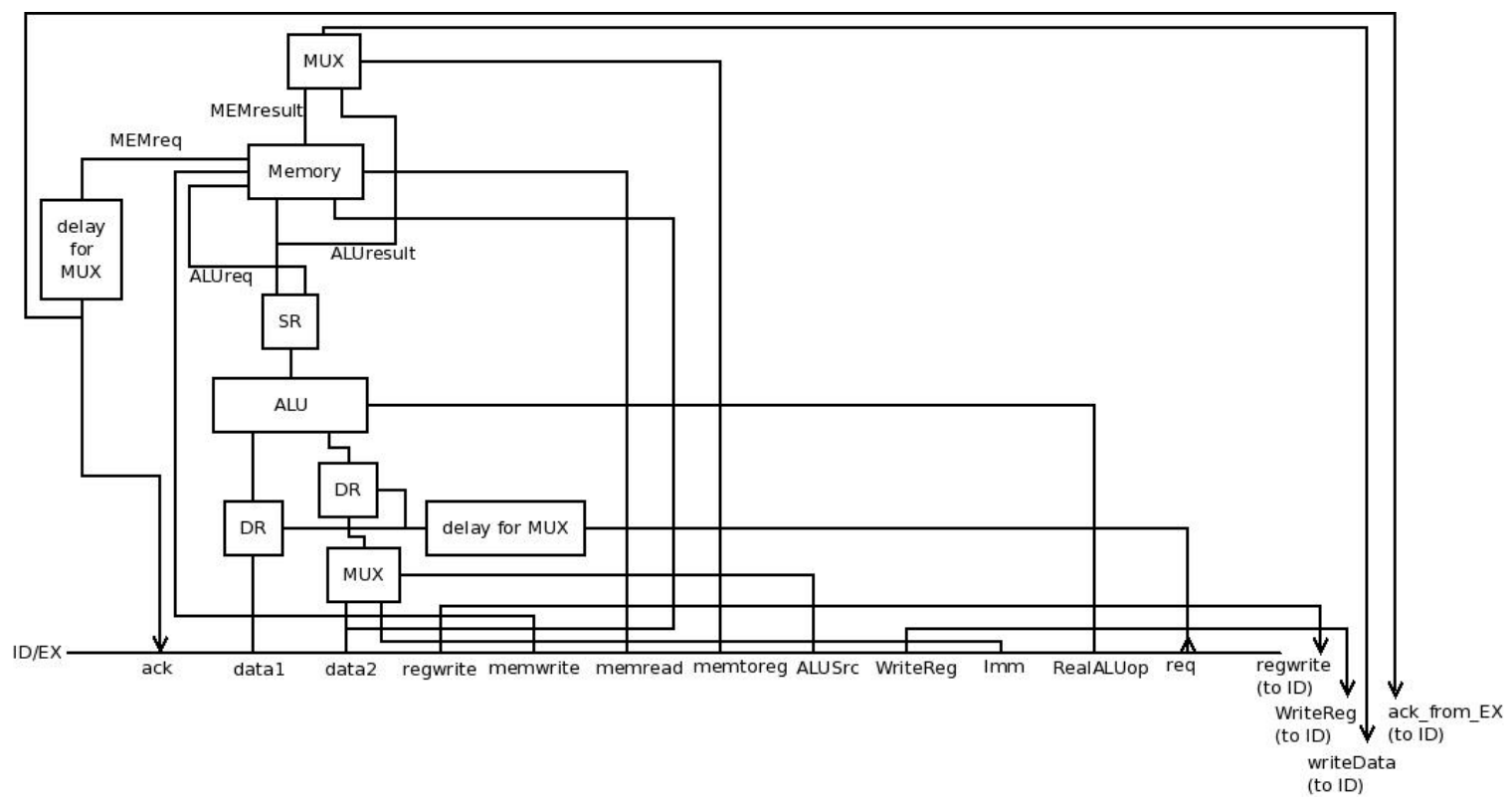

Figure 4.17: Execute Stage with wired for Memory Bypass

\subsubsection{ALU}

The basis of the ALU is a DIMS single bit adder module that is then used to create a ripple carry adder and ultimately a complete ALU. The truth table for the single bit adder is:

\begin{tabular}{c|c|c||c|c} 
cin & A & B & result & cout \\
\hline 0 & 0 & 0 & 0 & 0 \\
1 & 0 & 0 & 1 & 0 \\
0 & 1 & 0 & 1 & 0 \\
1 & 1 & 0 & 0 & 1 \\
0 & 0 & 1 & 1 & 0 \\
1 & 0 & 1 & 0 & 1 \\
0 & 1 & 1 & 0 & 1 \\
1 & 1 & 1 & 1 & 1
\end{tabular}

As there are 8 possible inputs there will be 8,3 input, $\mathrm{C}$ elements. The values for result have been generated in the usual way by combining together the minterms. However, there is an optimization for the cout values. If the cout values were determined in the usual way, then every operation done by an adder would require a cascade through all the bits because the cout of bit $n$ is the cin of bit $n+1$. This can be avoided by noticing that if a and $\mathrm{b}$ are both 0 , then the 


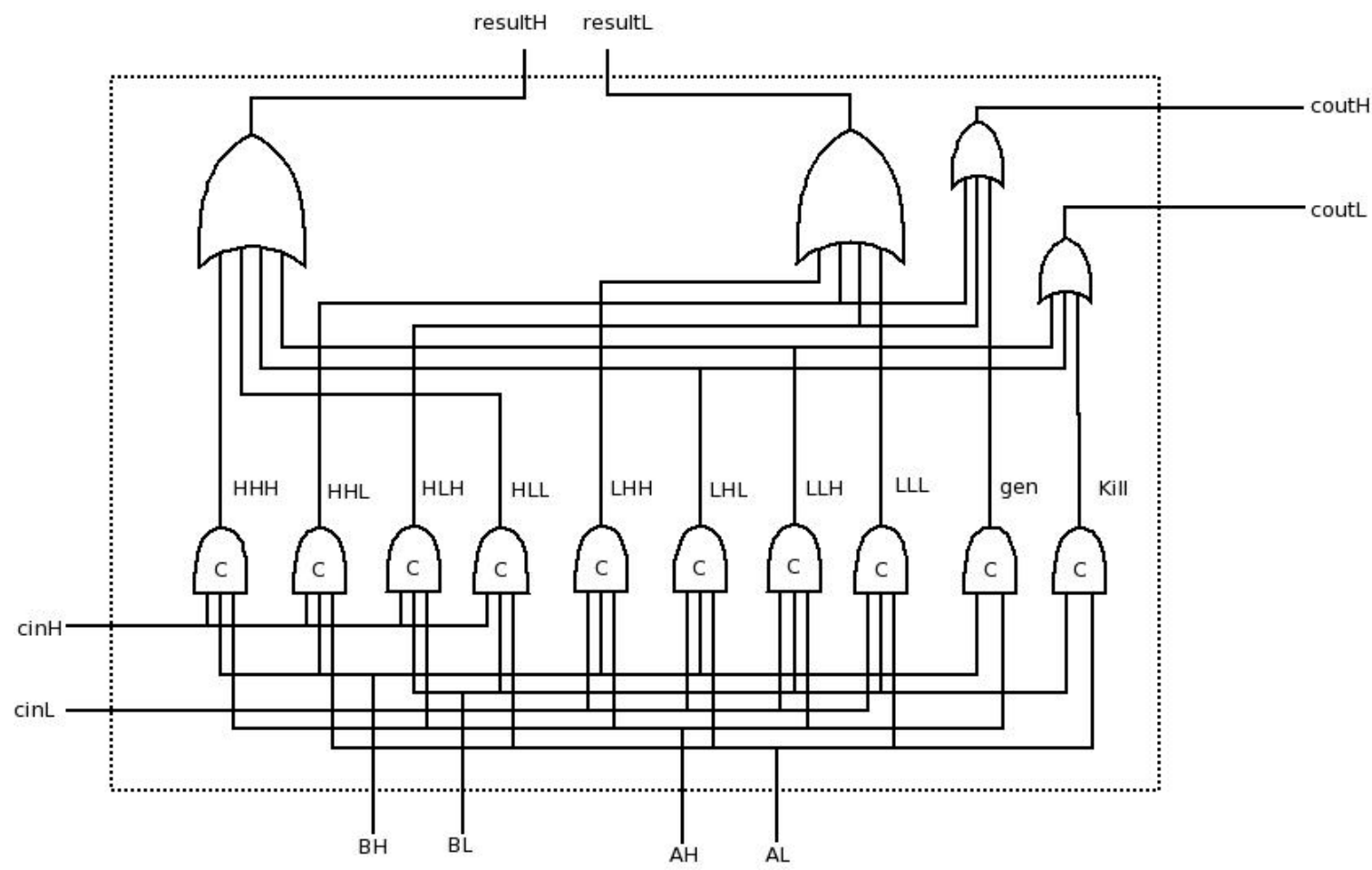

Figure 4.18: DIMS Adder with Generate and Kill carry

cout must be 0 so the adder has "killed the carry". Similarly, if both are 1, the cout must be 1 so the adder has "generated the carry". Creating these signals and combining them into the DIMS specification produces the circuit shown in figure 4.18. The source code for the adder module follows.

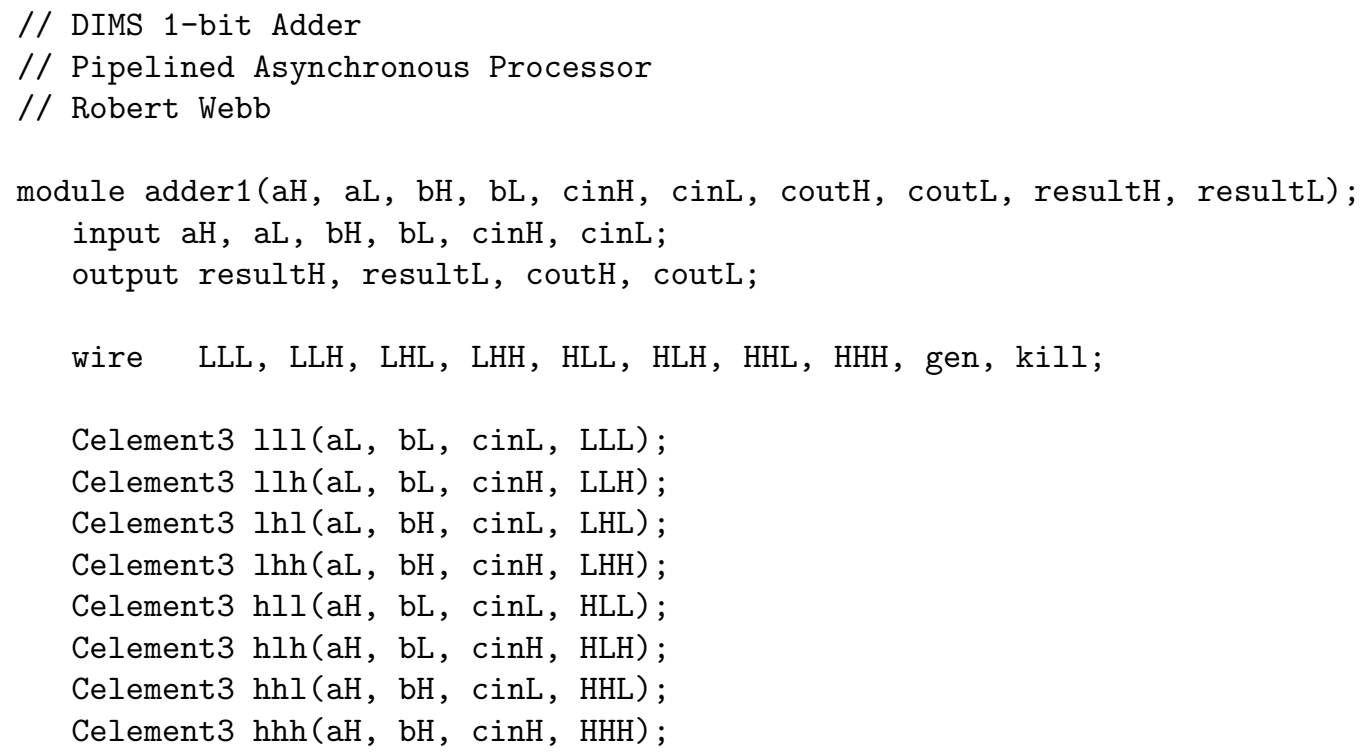




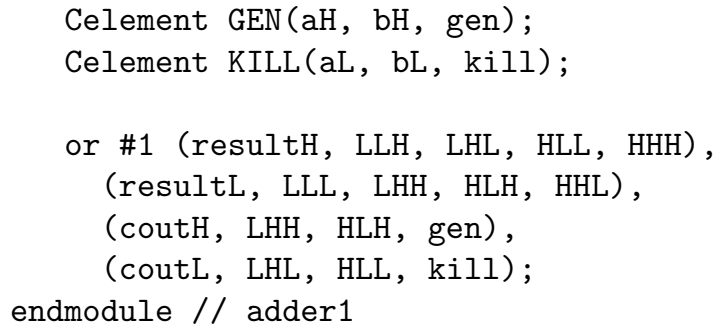

The ALU unit has several multiplexors to decide between the possible outputs of and, or, add, sub, slt. Because DIMS requires that all parts conform to the specification, behavioral code was used to make multiplexors that will return to the neutral state and have monotonic transitions. Structural code could be used to make these modules, but the selector for the multiplexor would have to be converted to dual-rail encoding as well, and the DIMS specification for a 4 input multiplexor would have $2^{5}=32 \mathrm{C}$ elements, each with 5 inputs.

\subsection{Results}

The pipelined asynchronous processor finished the program in 8335 time units. This was slightly slower than that single cycle processor described in chapter 3 , but that is not entirely unexpected. To create the asynchronous pipeline, a lot of overhead was created. The transistor count has greatly increased. Also, the processor in chapter 3 had very unrealistic timing assumptions with respect to IF and memory access. In the single cycle processor these things happened almost instantly, and the pipeline structure does not allow the information from memory accesses and IF accesses to be used instantly even if they are available. This should not be viewed as a bad thing because in exchange the processor is much more robust.

A possible way to speed up the circuit would be to use the two phase protocol introduced by Ivan Sutherland in his 1988 Turing Award Lecture.[13] In this protocol the request or acknowledge is communicated by a transition up or down on the channel. While this could be faster in certain circumstances because it negates the necessity of waiting for all the channels to return to 0 , it signifi- 
cantly increases circuit complexity, so was not used in this project because of the intended audience.

On a more theoretical note, one primary purpose of pipelining in a synchronous processor is to distribute the tasks that have a fixed delay so that they can be performed in advance while another instruction is performing an operation with variable delay. In a simple synchronous processor, the worst case of that variable delay usually dwarfs the fixed delays, and the clock must run at the worst case delay. In an asynchronous processor no units with variable delay run at their worst case delay except in very unusual circumstances. This would certainly lead to a diminished return for pipelining in an asynchronous setting.

Another factor that might explain the lack of speed increase is the test program. The loop in the test program is short, and the branch is taken almost every time. This would cause the branch delay slot to flush, losing potential instruction time. When combined with the fact that the pipeline has only 3 stages instead of the usual 5, and the loss of a slot every 7 instructions adds up over 20 iterations of a loop. 


\section{Chapter 5}

\section{Educational Resources}

The primary purpose of this project was to generate simulations of asynchronous processors for student use. An example of a synchronous single cycle simulation is presented as the starting point for this work in Appendix A. The basic single cycle asynchronous simulation code is presented in Appendix B. Finally the pipelined asynchronous simulation code is presented in Appendix C.

\subsection{Laboratory Exercises}

Descriptions of a series of laboratory exercises are included as appendix D. The modular nature of the simulation and the parallel with COD provides straightforward integration into a traditional course straightforward. The six lab exercises vary in difficulty and are also correlated with COD, so an instructor could choose to use as much or as little as desired. If an instructor wanted to add a single lecture and lab to expose students to the concepts of asynchronous design, an overview of the single cycle asynchronous processor in chapter 3 could be presented in lecture. The first suggested lab assignment does not involve any code, only manipulation of constants in existing code to demonstrate understanding of the delay models. 
If the instructor wanted to cover asynchronous logic design, another lecture and lab would be the only requirement. The DIMS procedure could be introduced in an hour lecture, assuming a solid understanding of traditional logic design. The second lab described in appendix D, a structural description of the control unit, is already in the curriculum of most architecture courses, and is assumed here. Applying the DIMS procedure as described in the third lab would require an additional $2^{6}=64$ lines of code to make the $\mathrm{C}$ elements. These first 64 lines of code would be almost identical. Most students will write the first one, make 63 copies, and modify the copies as required. Some particularly astute students may notice that the module is undefined for most of the 64 possible inputs, so many of those lines are not necessary. To complete the lab, 8 lines of code are necessary to connect the required outputs to the corresponding minterms, so the lab would have an upper bound of approximately 72 lines of code, depending on the optimizations that the students discover in the processor. If more practice with this procedure is required, labs 4 and 5 also convert various parts of the pipelined asynchronous processor using the DIMS procedure. These modules are less complicated than the control unit, so they would also have less than 72 lines of code. For those who want to cover pipelining, combining labs 3, 4, and 5 into a single assignment because of their common theme is advised. A combination of these labs would produce approximately 150 total lines of code, with an optimal solution requiring much less.

For an instructor to introduce the asynchronous pipeline design, an additional 2-3 lectures and 1-2 lab periods would be necessary. Assuming knowledge of the synchronous MIPS pipeline, the lectures would focus on the handshaking protocol, asynchronous pipeline control units, and integrating the constant delay and variable delay processing units into the pipeline. Lab 6 would then involve integrating the product of labs 3, 4, and 5 into the design. There would be very few lines of code required to accomplish this task, a good student will probably find a solution by adding fewer than 50 lines of code to the simulation, but a solid understanding of how the pipeline control and handshaking protocol function would be essential. 


\subsection{Notes on the use of these simulations}

The code for the simulations has been written in structural Verilog wherever possible to ensure the most accurate module of delay. Depending on the level of the class, some modules might be better understood if converted to a behavioral specification. For example, the register file unit is completely specified using

multiplexors and flip-flops, which would be beyond the level of most beginning computer architecture students outside of an electrical engineering program. 


\section{Chapter 6}

\section{Conclusions and Future Work}

\subsection{Conclusion}

This paper demonstrates that asynchronous techniques can be implemented using tools and techniques common in beginning computer architecture courses. While not trivial by any means, the ideas presented in this report have a certain spark that could lead to many teachable moments. Additionally, grasping asynchronous concepts could greatly add to students overall understanding of the synchronous ideas in COD. The traditional architecture instructor could integrate these ideas into a class by adding as little as a single lecture and experimental lab with no code. To go into great depth, adding 4-6 lectures and 3-4 labs to the curriculum would integrate the breath of topics presented in this paper into a traditional course while adding as little as 150-200 lines of code to the workload of a capable student. Because many architecture students, over the course of a term, produce a processor simulation similar to the one presented in appendix A and containing 778 lines of code, this would be an increase of approximately $20-25 \%$. 


\subsection{Future Work}

This project provides substantial avenues for future work.

- Decompose the EX stage into multiple, concurrent micro execution stages.

- Dynamic Scheduling to take advantage of concurrent micro execution stages

- Set up the register file for multiple asynchronous write back values

- Implement forwarding if other modifications make it necessary.

- Implement memory hierarchy and waiting for cache misses.

- Replace behavioral code with structural code.

- Implement more of the MIPS instruction set

- Multiprocessor applications

- Modeling Power consumption differences 


\section{Bibliography}

[1] A. Davis and S. Nowick, An Introduction to Asynchronous Circuit Design, University of Utah, Salt Lake City, 1997.

[2] R. Manohar, A. J. Martin, Quasi-delay-insensitive circuits are Turingcomplete, Second International Symposium on Advanced Research in Asynchronous Circuits and Systems, 1995.

[3] A. J. Martin, et al, The Design of an Asynchronous Microprocessor, Proc. Decennial Caltech Conference on VSLI, Pasadena, 1989.

[4] A. J. Martin, et al, The First Asynchronous Microprocessor: The Test Results, ACM SIGARCH Computer Architecture News, 17(4): 95-98, June 1989.

[5] A. J. Martin, Tomorrow's Digital Hardware will be Asynchronous and Verified, Proceedings of the IFIP 12th World Computer Congress on Algorithms, Software, Architecture - information Processing '92, 1(1): 684-695, 1992.

[6] A. J. Martin, Asynchronous Datapaths and the Design of an Asynchronous Adder, Cal Formal Methods in System Design: 119-137, 1991.

[7] A. J. Martin, The Limitations to Delay-Insensitivity in Asynchronous Circuits, Proceedings of the 6th MIT Conference on Advanced Research in VSLI, MIT Press, Cambridge, 1990.

[8] A. J. Martin, et al, The Design of an Asynchronous MIPS R3000 Microprocessor, Proceedings of the Seventeenth Conference on Advanced Research in VLSI, 1997. 
[9] A. J. Martin, et al, Speed and Energy Performance of an Asynchronous MIPS R3000 Microprocessor, Technical Report, Cal Tech Computer Science Department, Pasadena, 2001.

[10] D. E. Muller and W. S. Bartky, A Theory of Asynchronous Circuits, Proc. Int'l Symp. Theory of Switching, Part 1, Harvard Univ. Press, 1959, pp. 204243.

[11] D. A. Patterson, J. L. Hennessy, Computer Organization 83 Design: The Hardware-Software Interface, Second Edition, Morgan Kaufmann, San Francisco, 1998.

[12] J. Sparsø, Asynchronous Circuit Design: A Tutorial, Technical University of Denmark, 2006.

[13] I. E. Sutherland, Micropipelines, Communications of the ACM, 32(6): 720 738, June 1989.

[14] J. M. Yarbrough, Digital Logic: Applications and Design, PWS Publishing, Boston, 1997. 


\section{Appendix A}

\section{The Complete Synchronous}

\section{Single-Cycle Source Code}

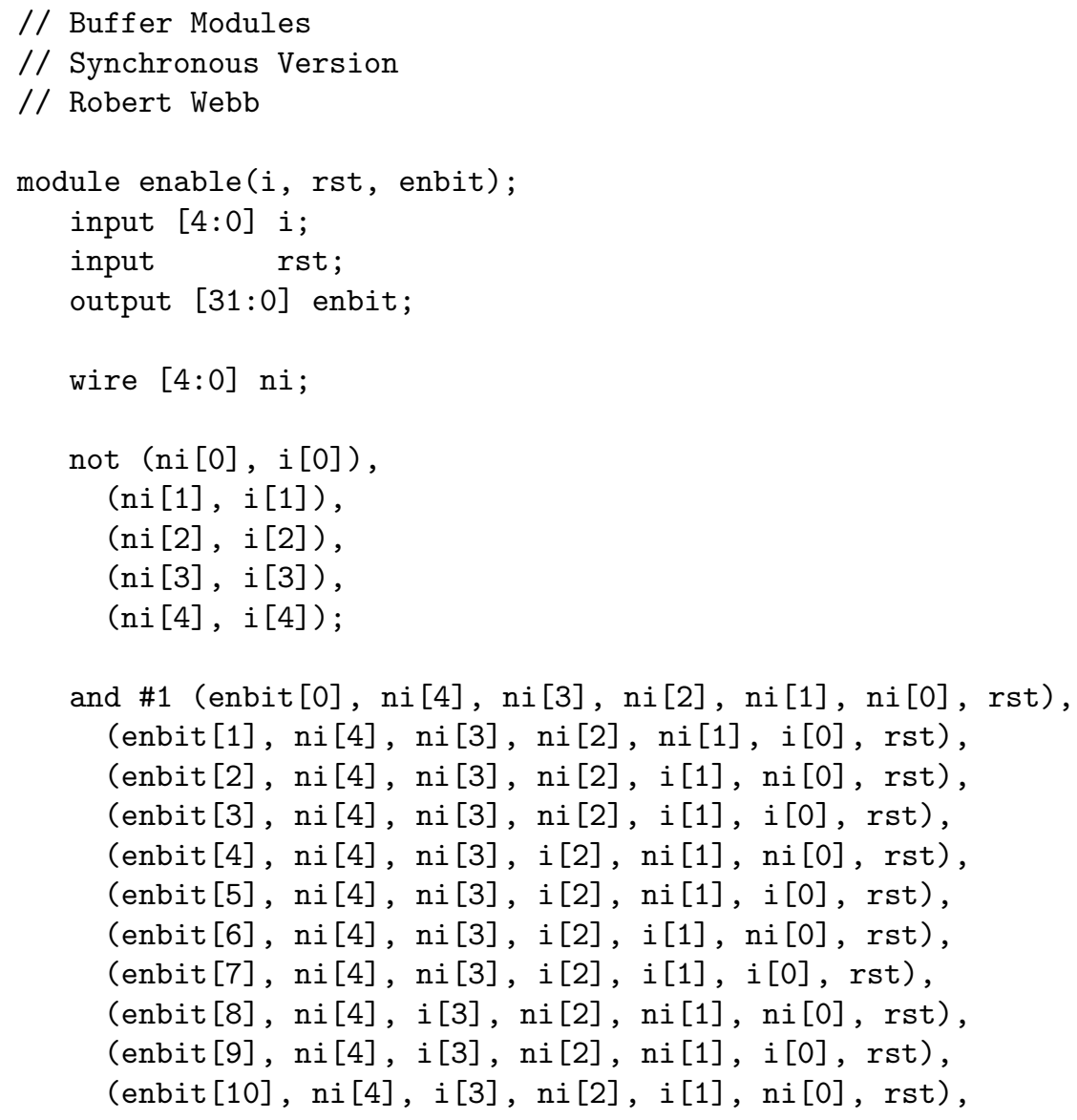




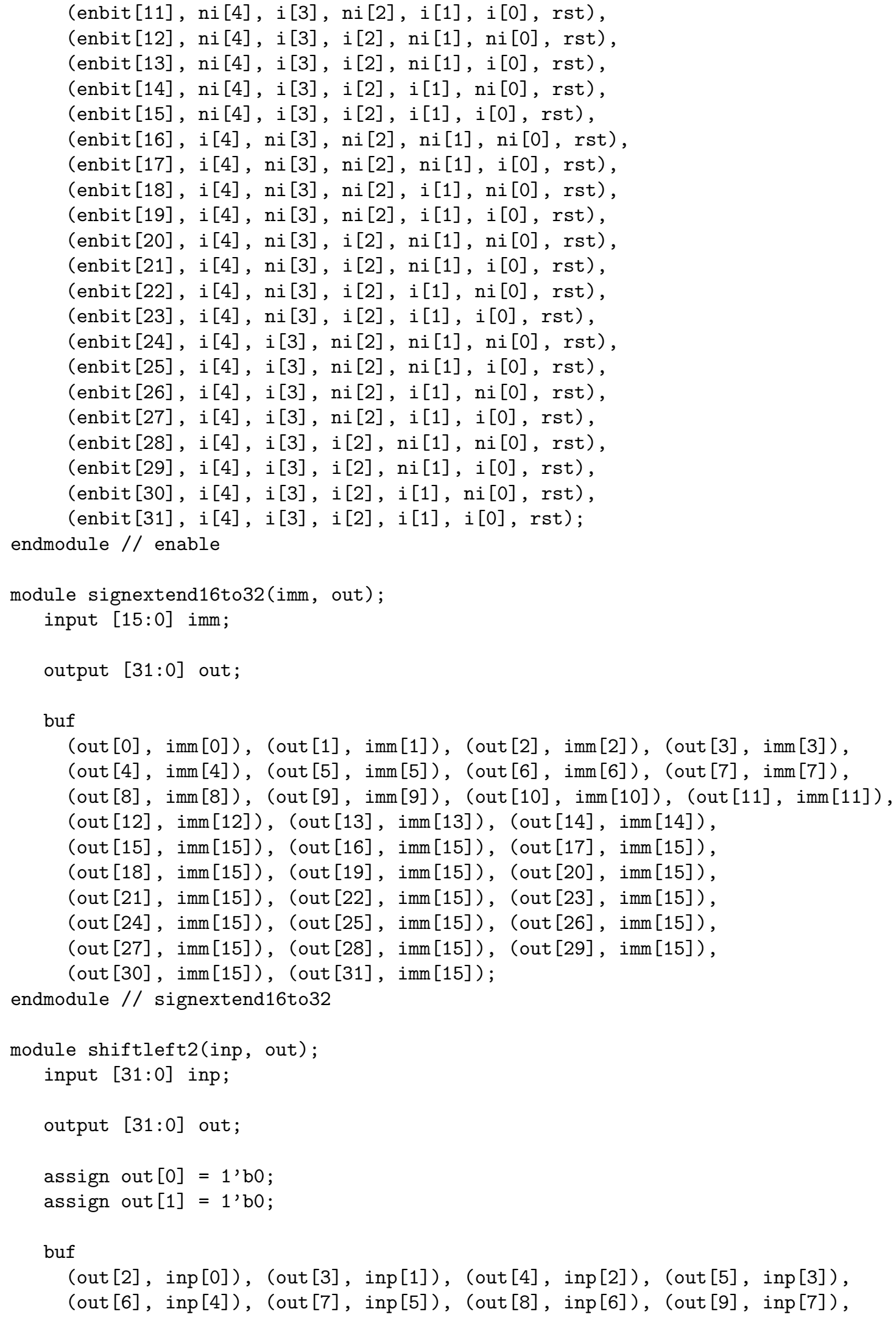




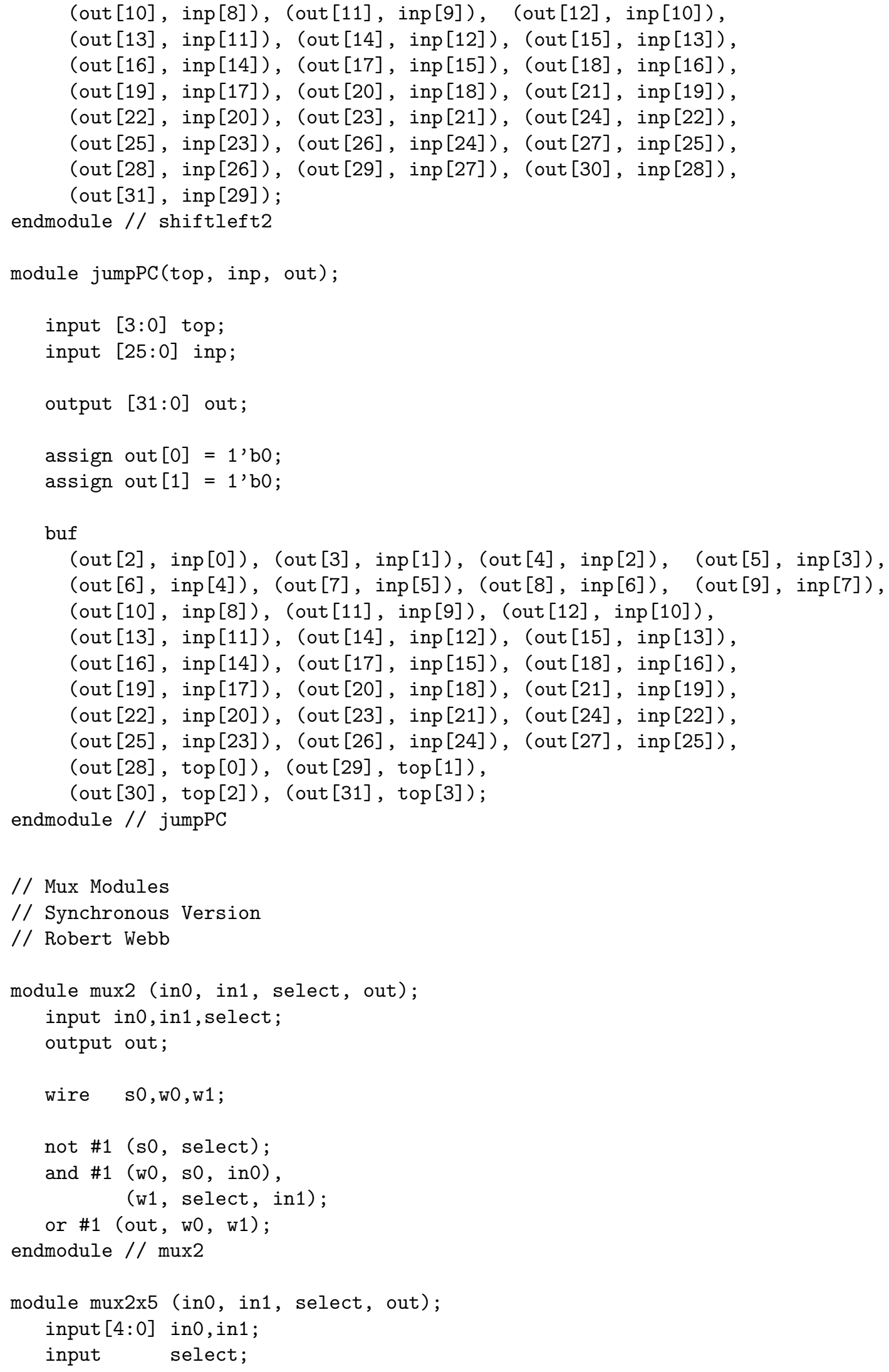




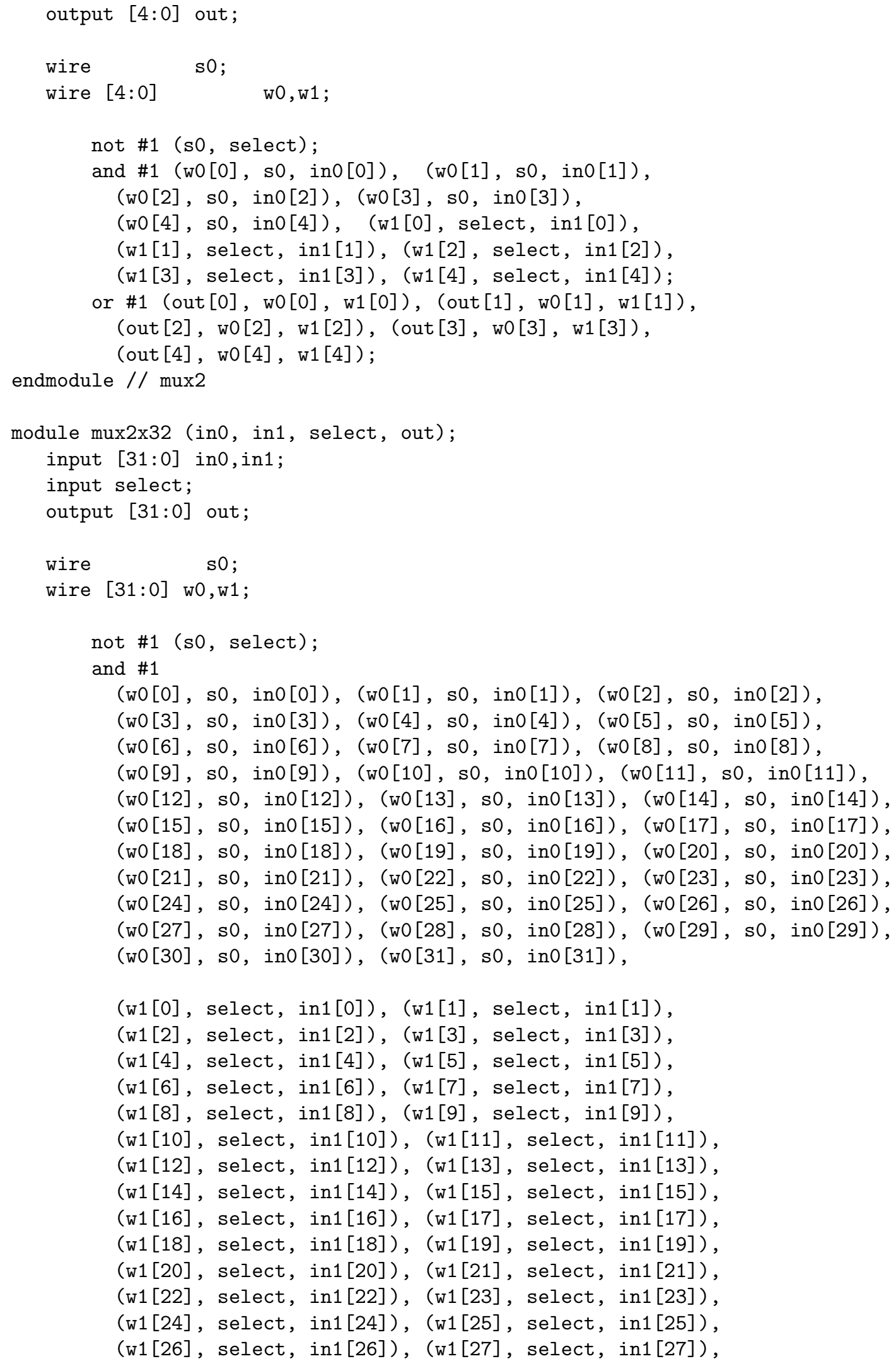




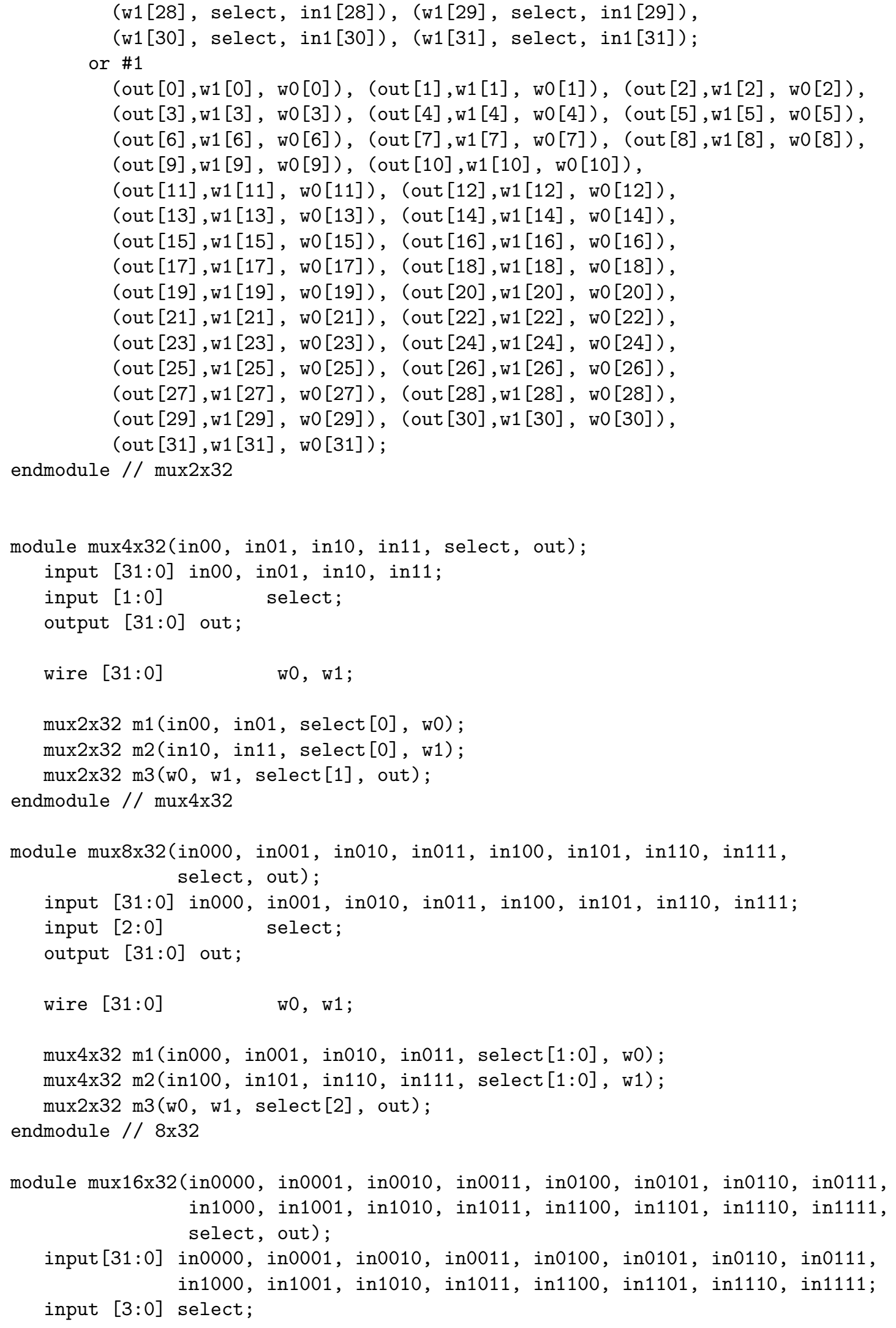




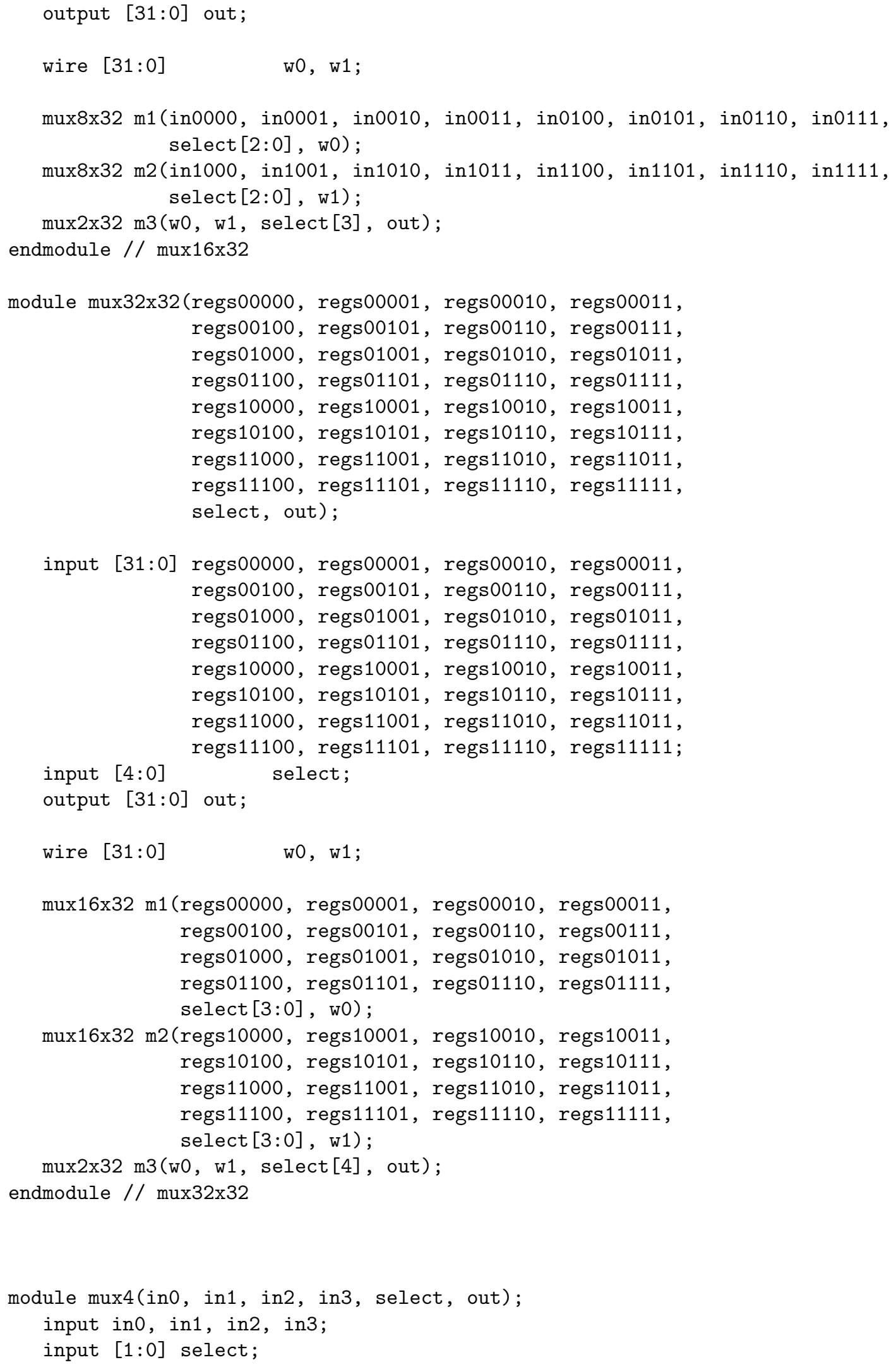




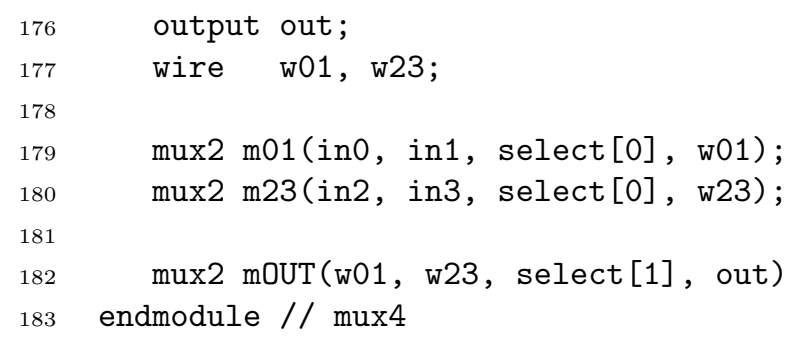




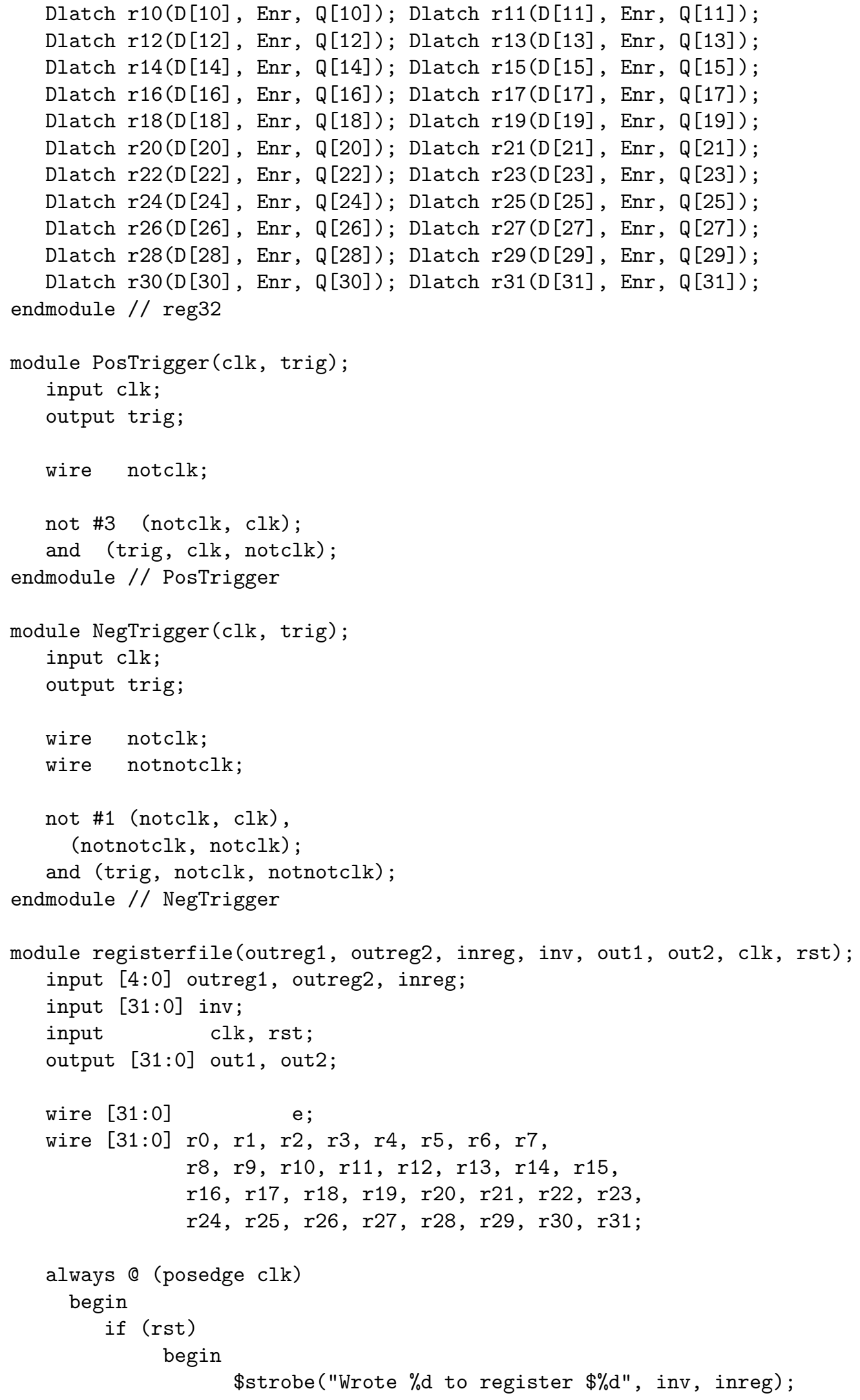




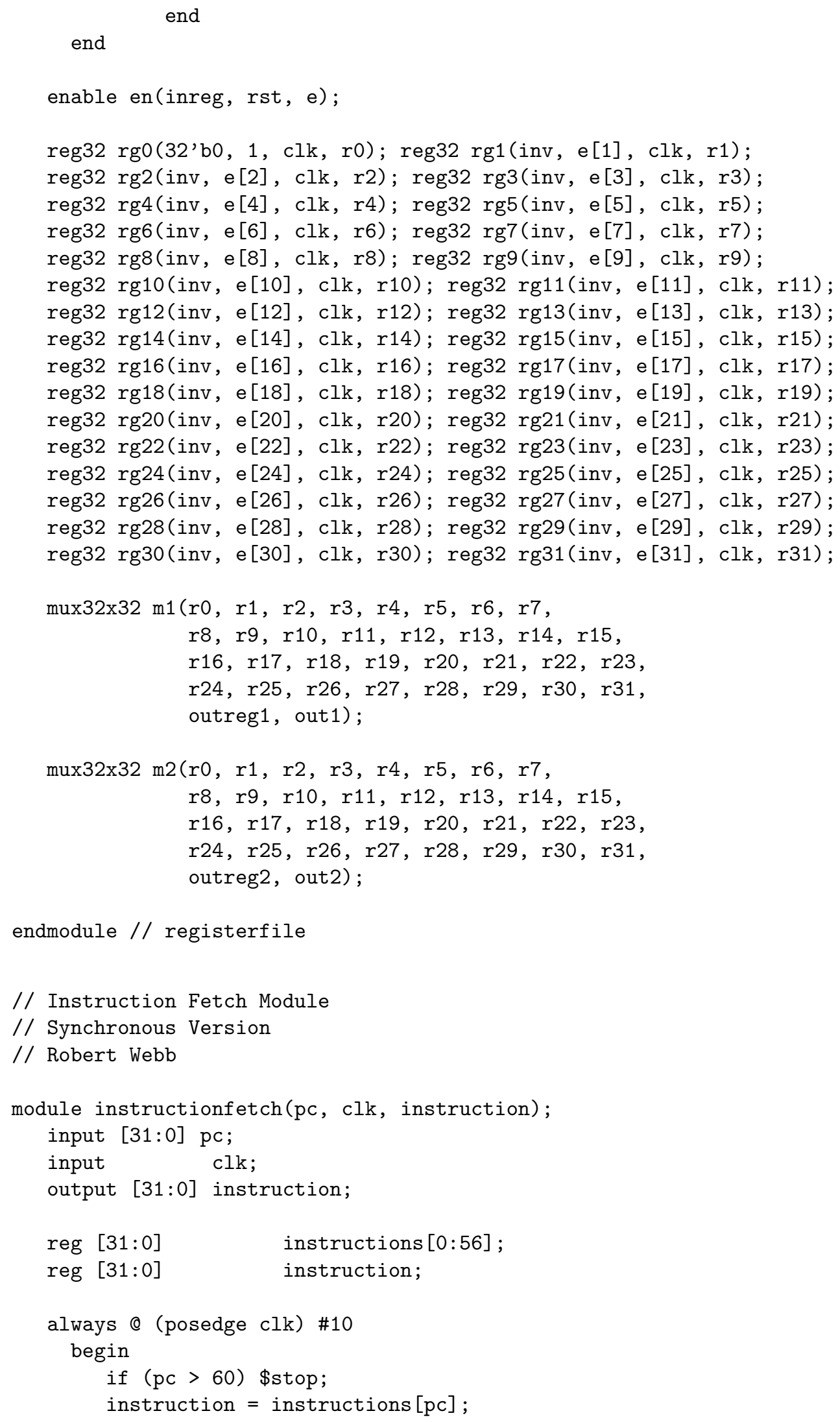




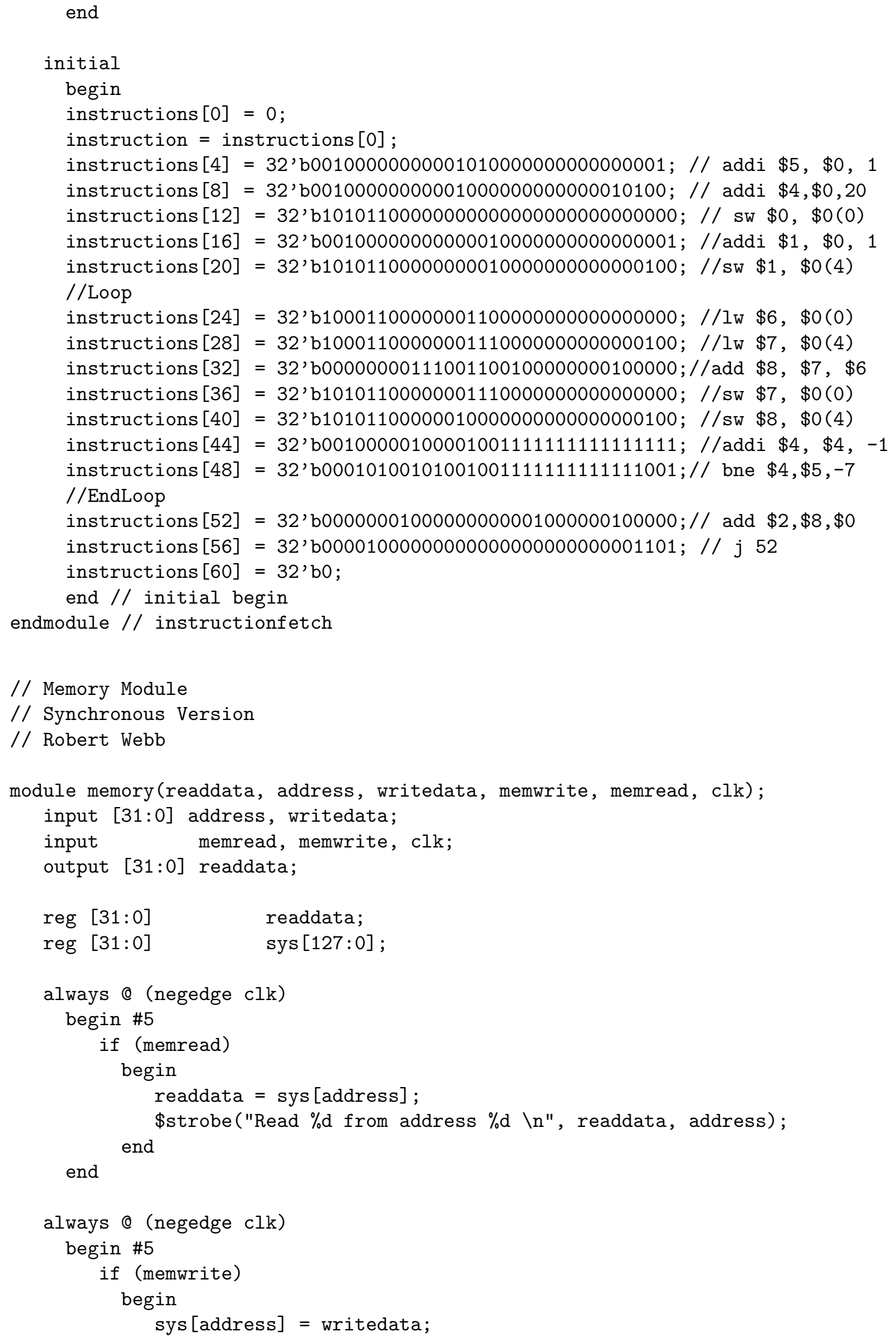




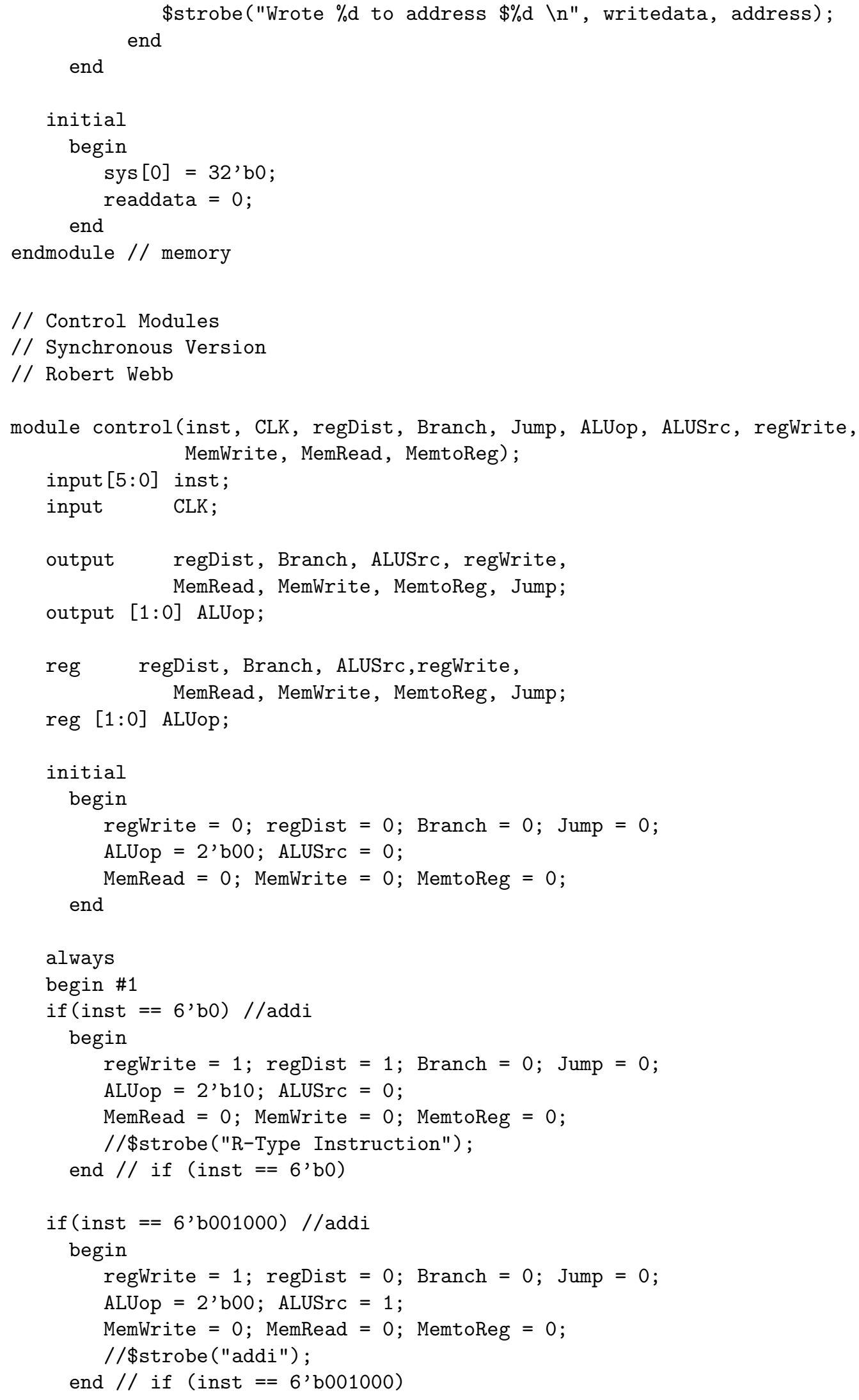




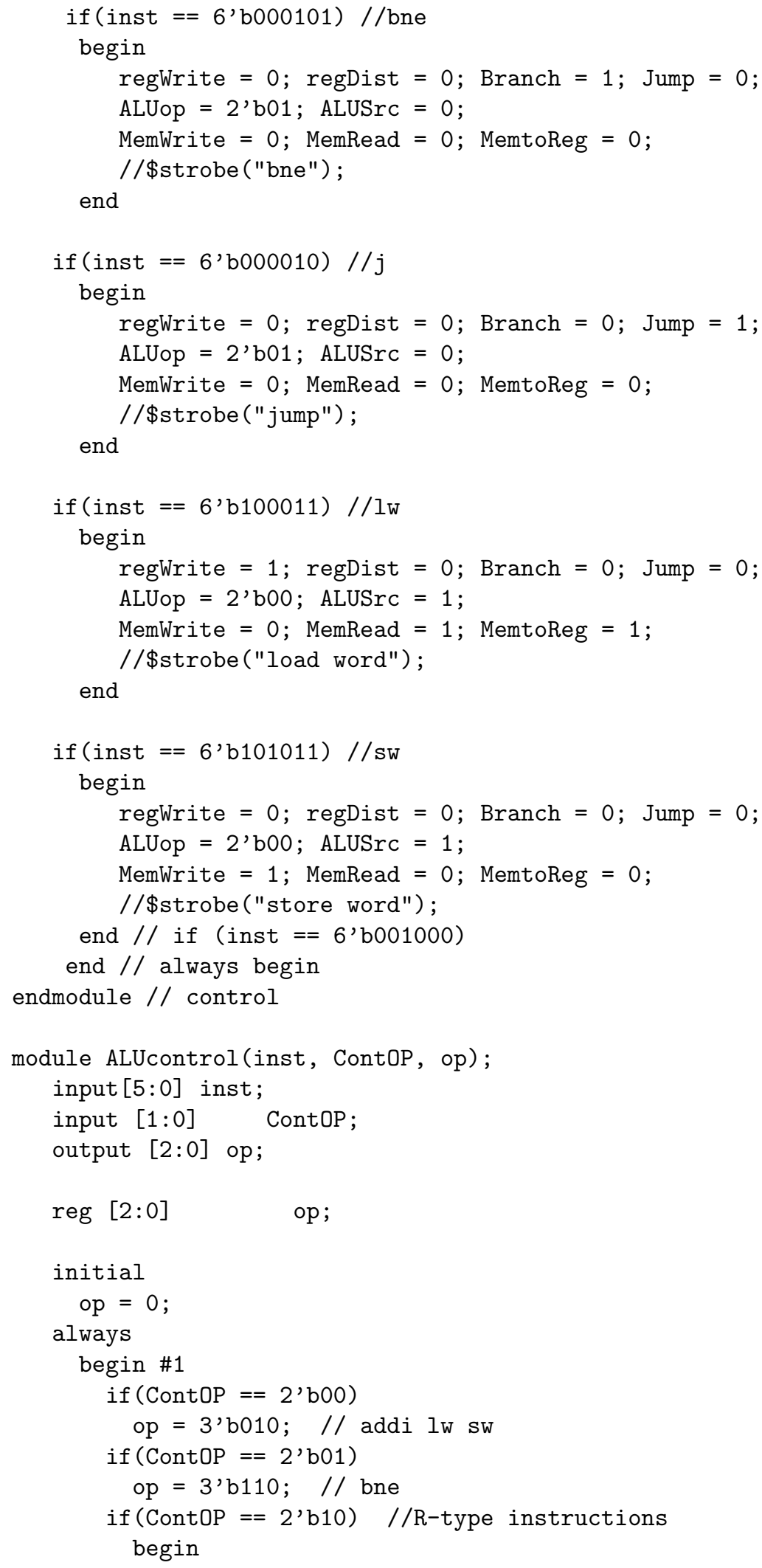




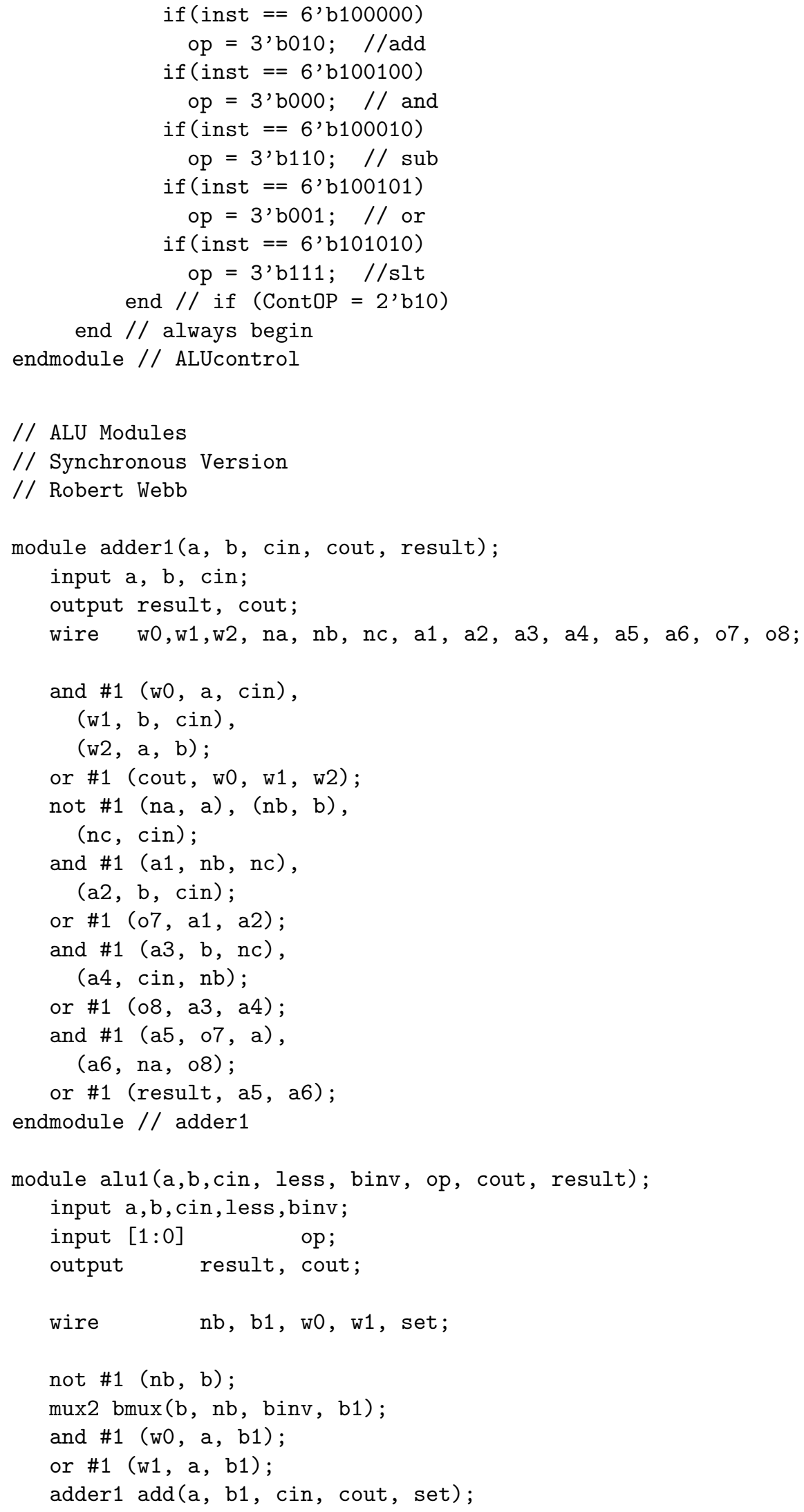




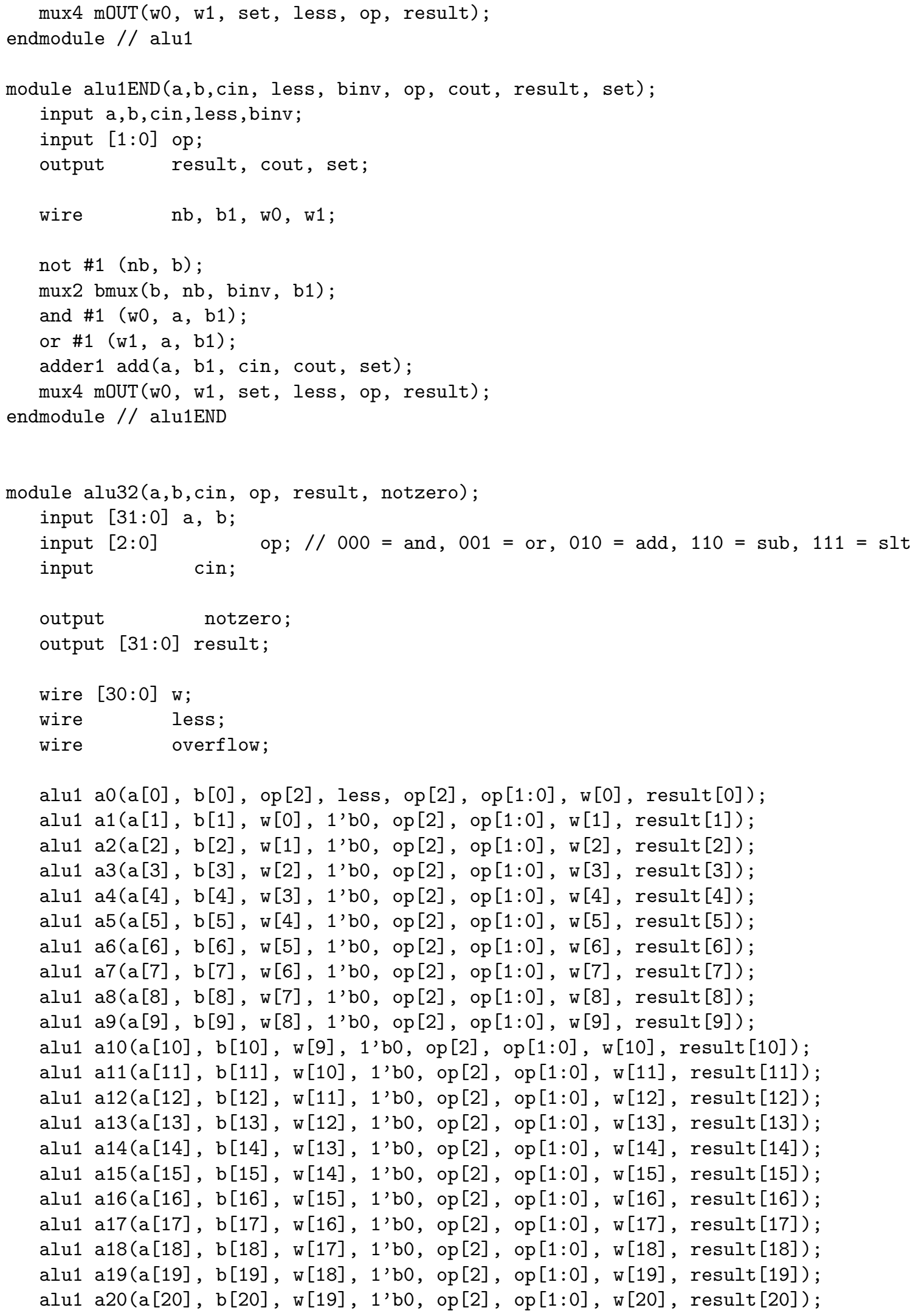




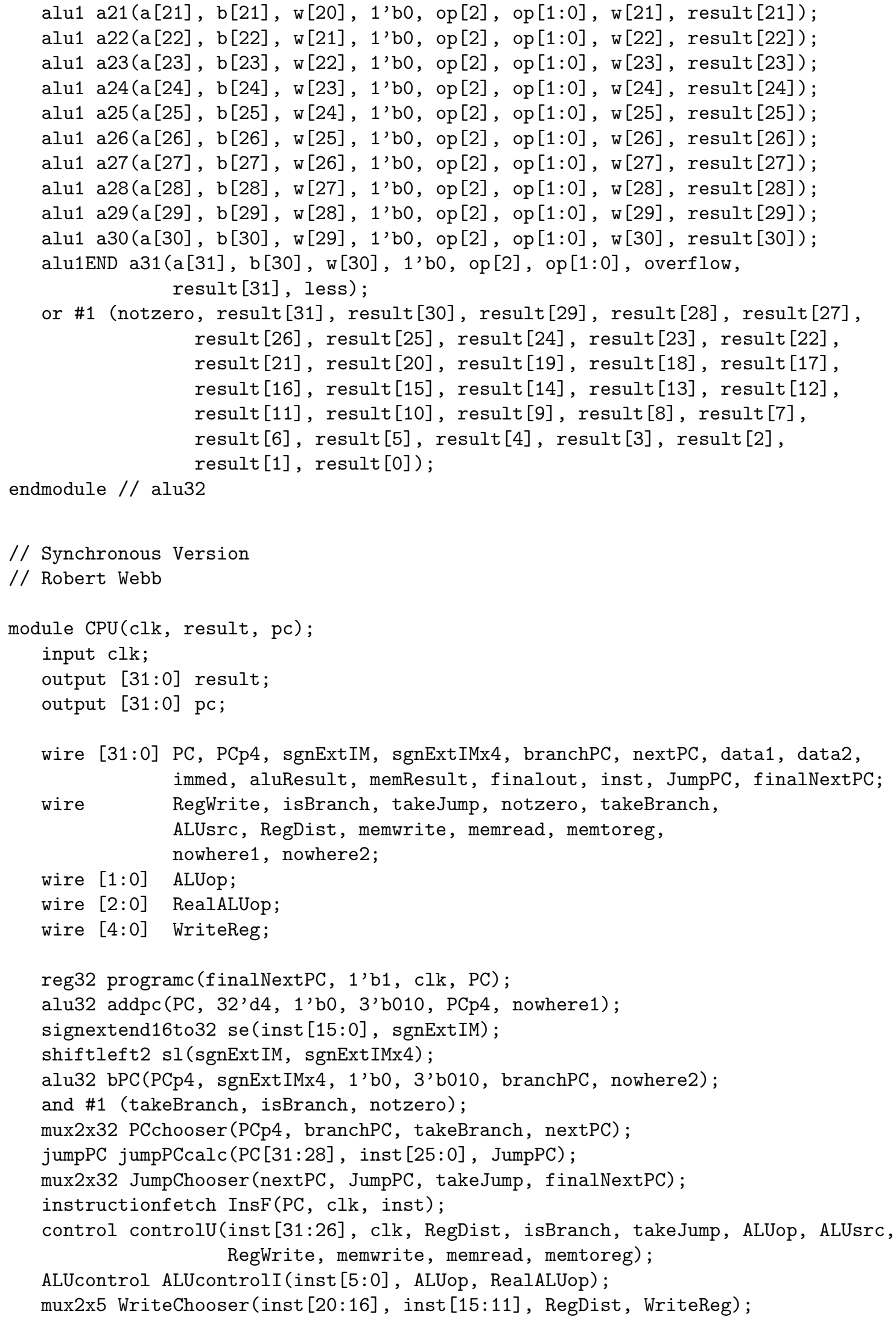




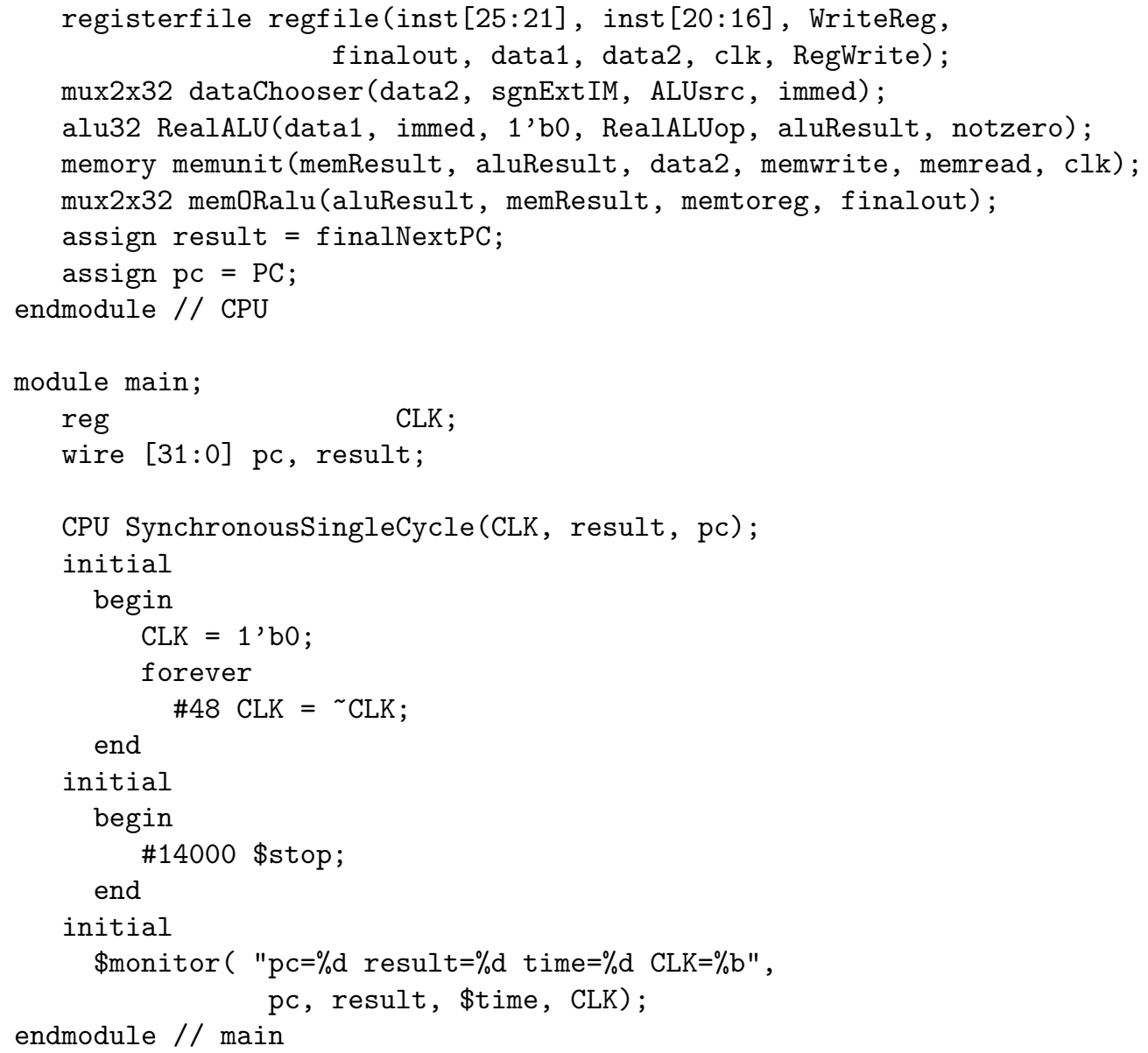




\section{Appendix B}

\section{The Complete Single-Cycle}

\section{Asynchronous Source Code}

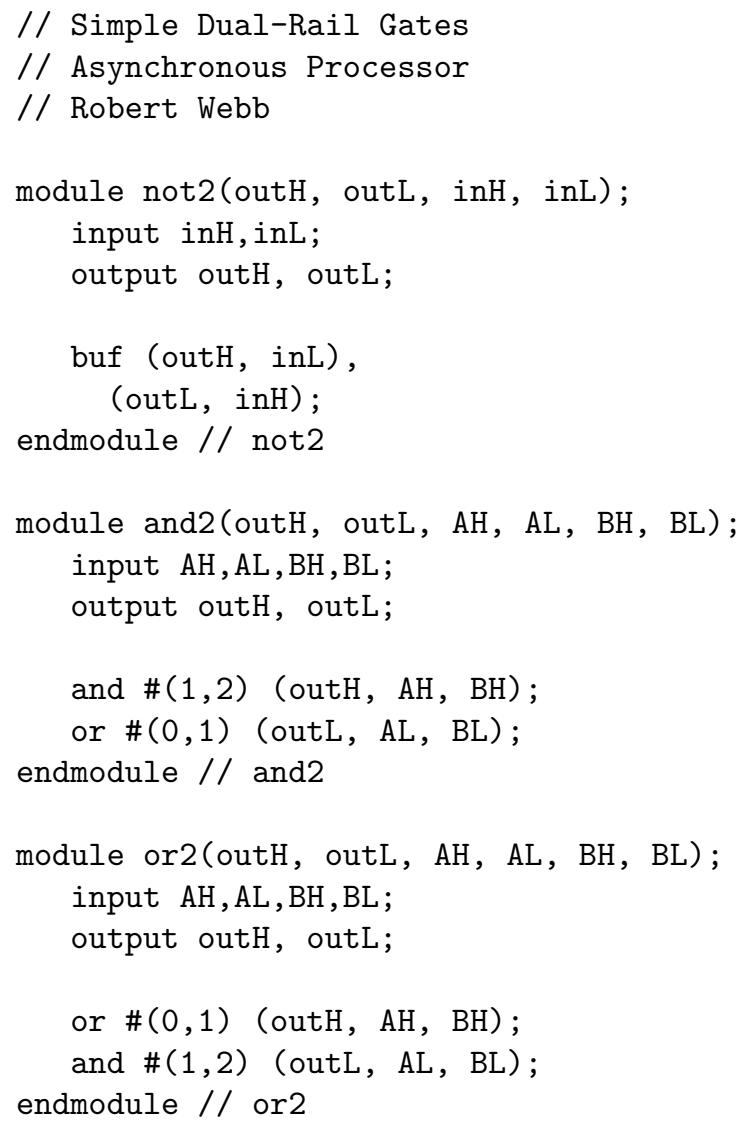




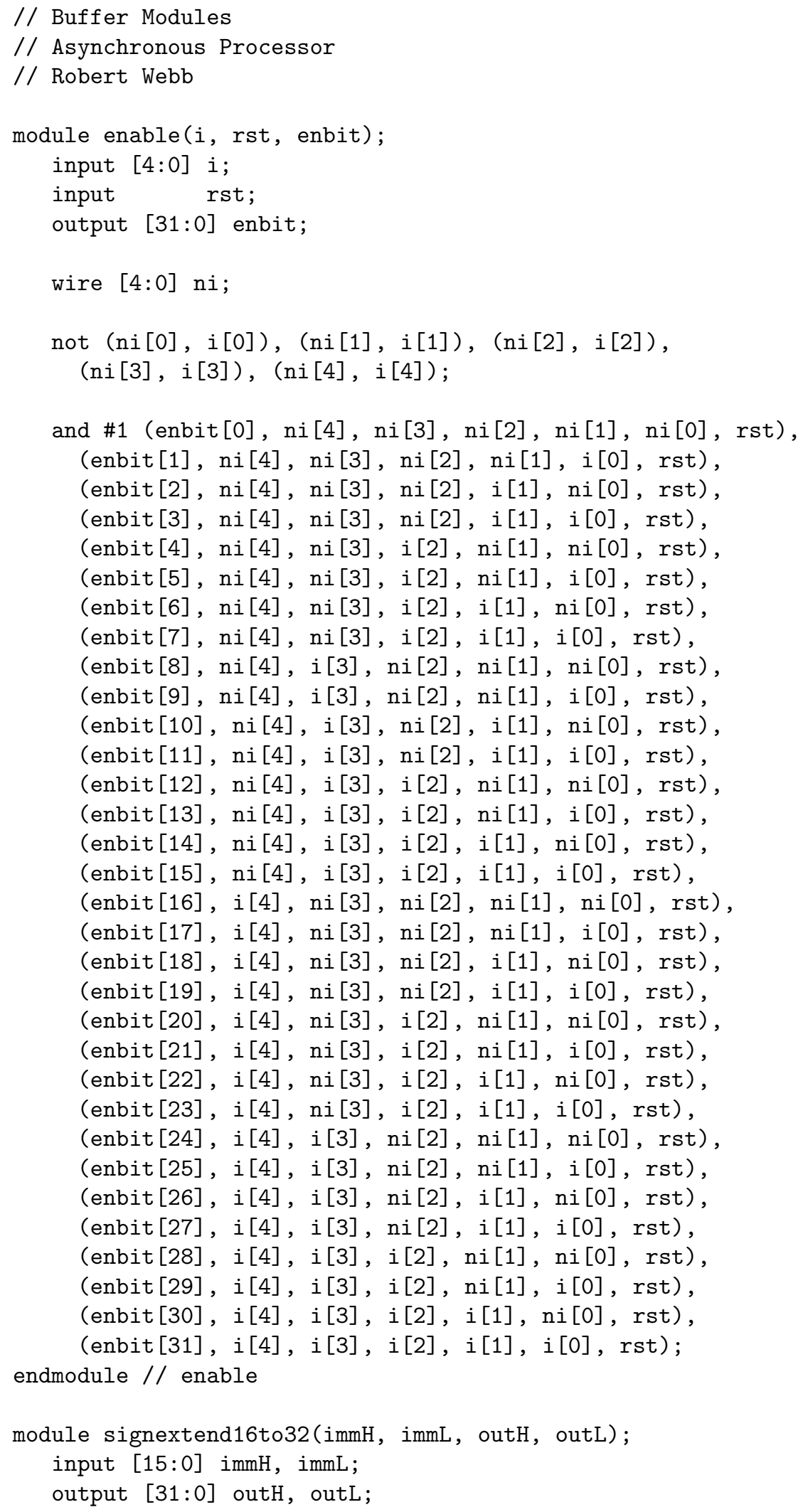




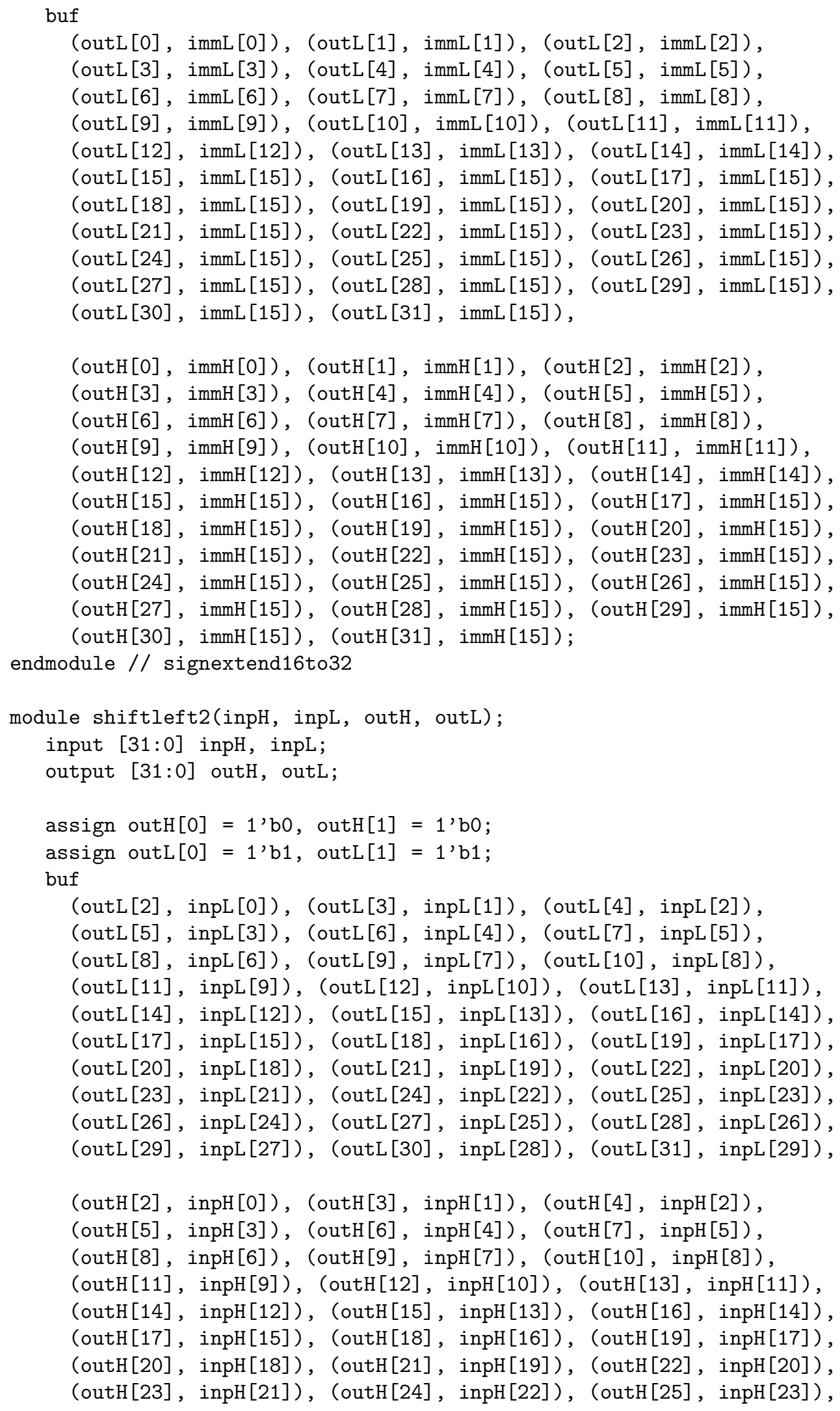




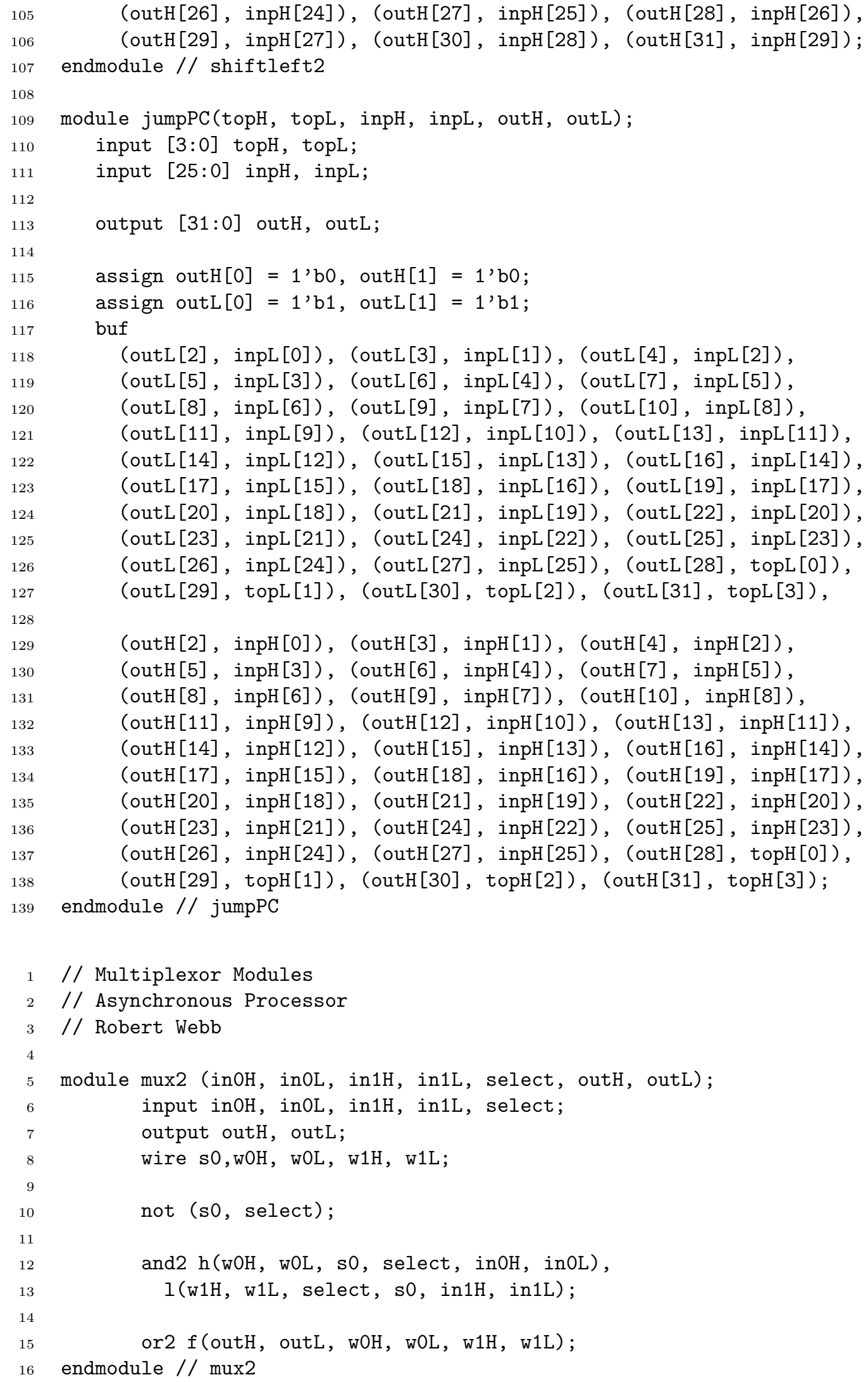




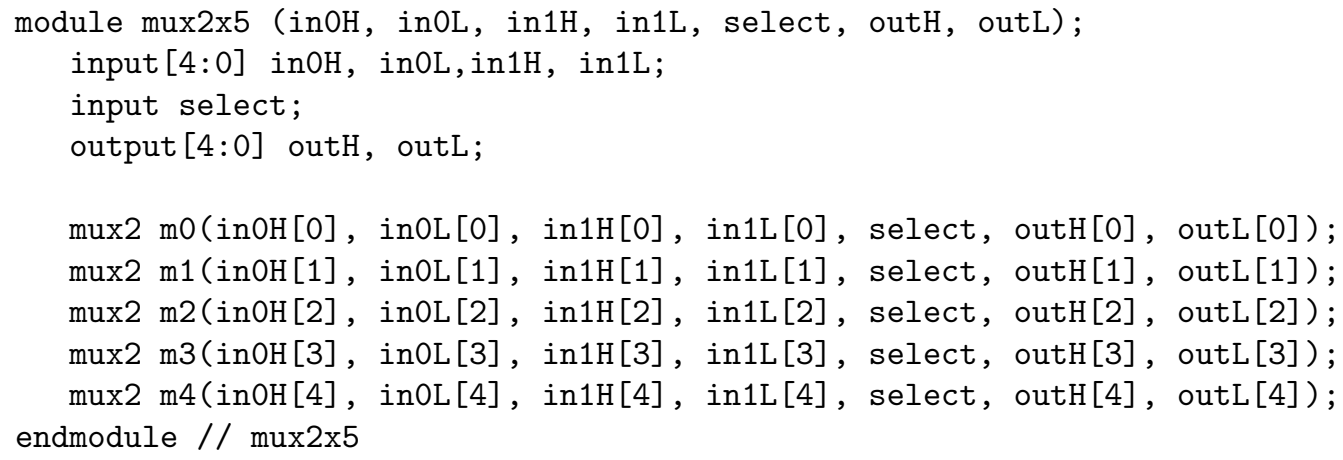




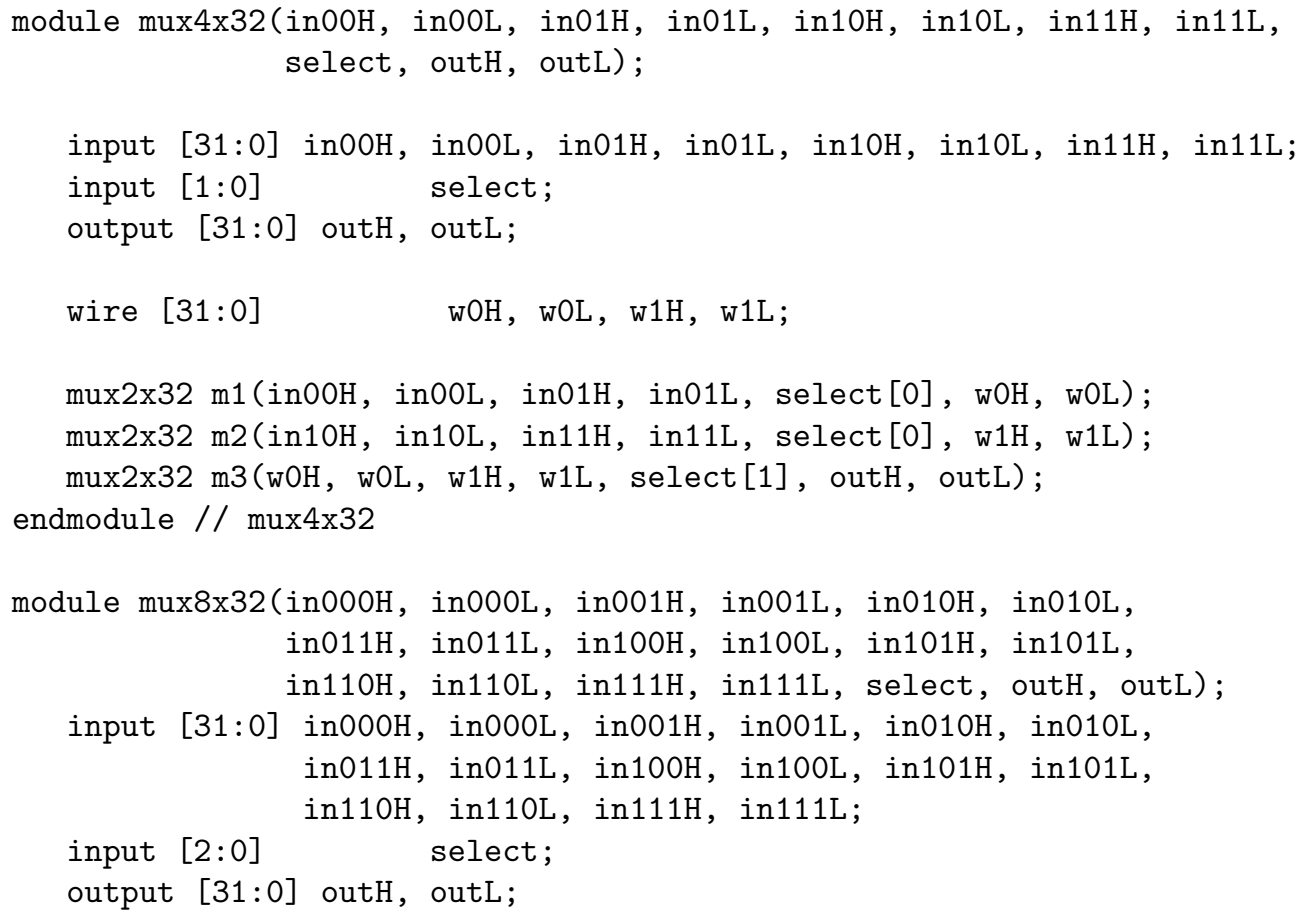


in $0110 \mathrm{H}$, in0110L, in 0111H, in0111L, select [2:0], w0H, w0L); mux8x32 $\mathrm{m} 2($ in $1000 \mathrm{H}$, in $1000 \mathrm{~L}$, in $1001 \mathrm{H}$, in $1001 \mathrm{~L}$, in $1010 \mathrm{H}$, in $1010 \mathrm{~L}$, in $1011 \mathrm{H}$, in $1011 \mathrm{~L}$, in $1100 \mathrm{H}$, in1100L, in1101H, in1101L, in $1110 \mathrm{H}$, in1110L, in1111H, in1111L, select [2:0], w1H, w1L); $\operatorname{mux} 2 \times 32 \mathrm{~m} 3(\mathrm{wOH}$, wOL, w1H, w1L, select[3], outH, outL); endmodule // $\operatorname{mux} 16 \times 32$

module $\operatorname{mux} 32 \times 32(i n 00000 \mathrm{H}$, in $00000 \mathrm{~L}$, in $00001 \mathrm{H}$, in $00001 \mathrm{~L}$, in $00010 \mathrm{H}$, in $00010 \mathrm{~L}$, in $00011 \mathrm{H}$, in $00011 \mathrm{~L}$, in $00100 \mathrm{H}$, in $00100 \mathrm{~L}$, in $00101 \mathrm{H}$, in $00101 \mathrm{~L}$, in $00110 \mathrm{H}$, in $00110 \mathrm{~L}$, in $00111 \mathrm{H}$, in $00111 \mathrm{~L}$, in $01000 \mathrm{H}$, in $01000 \mathrm{~L}$, in $01001 \mathrm{H}$, in $01001 \mathrm{~L}$, in $01010 \mathrm{H}$, in $01010 \mathrm{~L}$, in $01011 \mathrm{H}$, in $01011 \mathrm{~L}$, in $01100 \mathrm{H}$, in $01100 \mathrm{~L}$, in $01101 \mathrm{H}$, in $01101 \mathrm{~L}$, in $01110 \mathrm{H}$, in $01110 \mathrm{~L}$, in $01111 \mathrm{H}$, in $01111 \mathrm{~L}$, in $10000 \mathrm{H}$, in $10000 \mathrm{~L}$, in $10001 \mathrm{H}$, in $10001 \mathrm{~L}$, in $10010 \mathrm{H}$, in $10010 \mathrm{~L}$, in $10011 \mathrm{H}$, in10011L, in10100H, in10100L, in $10101 \mathrm{H}$, in $10101 \mathrm{~L}$, in $10110 \mathrm{H}$, in $10110 \mathrm{~L}$, in $10111 \mathrm{H}$, in $10111 \mathrm{~L}$, in $11000 \mathrm{H}$, in11000L, in11001H, in11001L, in11010H, in11010L, in $11011 \mathrm{H}$, in $11011 \mathrm{~L}$, in $11100 \mathrm{H}$, in11100L, in11101H, in11101L, in $11110 \mathrm{H}$, in11110L, in $11111 \mathrm{H}$, in11111L, select, outH, outL); input [31:0] in $00000 \mathrm{H}$, in $00000 \mathrm{~L}$, in $00001 \mathrm{H}$, in $00001 \mathrm{~L}$, in $00010 \mathrm{H}$, in $00010 \mathrm{~L}$, in $00011 \mathrm{H}$, in $00011 \mathrm{~L}$, in $00100 \mathrm{H}$, in $00100 \mathrm{~L}$, in $00101 \mathrm{H}$, in $00101 \mathrm{~L}$, in $00110 \mathrm{H}$, in $00110 \mathrm{~L}$, in $00111 \mathrm{H}$, in $00111 \mathrm{~L}$, in $01000 \mathrm{H}$, in $01000 \mathrm{~L}$, in $01001 \mathrm{H}$, in $01001 \mathrm{~L}$, in $01010 \mathrm{H}$, in $01010 \mathrm{~L}$, in $01011 \mathrm{H}$, in $01011 \mathrm{~L}$, in $01100 \mathrm{H}$, in $01100 \mathrm{~L}$, in $01101 \mathrm{H}$, in $01101 \mathrm{~L}$, in $01110 \mathrm{H}$, in01110L, in $01111 \mathrm{H}$, in $01111 \mathrm{~L}$, in $10000 \mathrm{H}$, in $10000 \mathrm{~L}$, in $10001 \mathrm{H}$, in $10001 \mathrm{~L}$, in $10010 \mathrm{H}$, in10010L, in $10011 \mathrm{H}$, in $10011 \mathrm{~L}$, in $10100 \mathrm{H}$, in $10100 \mathrm{~L}$, in $10101 \mathrm{H}$, in $10101 \mathrm{~L}$, in $10110 \mathrm{H}$, in10110L, in10111H, in10111L, in $11000 \mathrm{H}$, in11000L, in11001H, in11001L, in11010H, in11010L, in $11011 \mathrm{H}$, in $11011 \mathrm{~L}$, in11100H, in11100L, in11101H, in11101L, in $11110 \mathrm{H}$, in $11110 \mathrm{~L}$, in $11111 \mathrm{H}$, in $11111 \mathrm{~L}$; in $00011 \mathrm{H}$, in $00011 \mathrm{~L}$, in $00100 \mathrm{H}$, in $00100 \mathrm{~L}$, in $00101 \mathrm{H}$, in $00101 \mathrm{~L}$, in $00110 \mathrm{H}$, in $00110 \mathrm{~L}$, in $00111 \mathrm{H}$, in $00111 \mathrm{~L}$, in $01000 \mathrm{H}$, in $01000 \mathrm{~L}$, in $01001 \mathrm{H}$, in $01001 \mathrm{~L}$, in $01010 \mathrm{H}$, in01010L, in01011H, in01011L, in $01100 \mathrm{H}$, in $01100 \mathrm{~L}$, in $01101 \mathrm{H}$, in $01101 \mathrm{~L}$, in $01110 \mathrm{H}$, in01110L, in $01111 \mathrm{H}$, in01111L, select [3:0], wOH, wOL);

$\operatorname{mux} 16 \times 32 \mathrm{~m} 2($ in $10000 \mathrm{H}$, in $10000 \mathrm{~L}$, in $10001 \mathrm{H}$, in10001L, in10010H, in10010L, in $10011 \mathrm{H}$, in $10011 \mathrm{~L}$, in $10100 \mathrm{H}$, in $10100 \mathrm{~L}$, in $10101 \mathrm{H}$, in $10101 \mathrm{~L}$, in $10110 \mathrm{H}$, in $10110 \mathrm{~L}$, in $10111 \mathrm{H}$, in $10111 \mathrm{~L}$, in $11000 \mathrm{H}$, in $11000 \mathrm{~L}$, in $11001 \mathrm{H}$, in $11001 \mathrm{~L}$, in $11010 \mathrm{H}$, in11010L, in11011H, in11011L, in11100H, in11100L, in11101H, in11101L, in11110H, in11110L, in $11111 \mathrm{H}$, in $11111 \mathrm{~L}$, select $[3: 0]$, w1H, w1L);

$\operatorname{mux} 2 \times 32 \mathrm{~m} 3(\mathrm{wOH}$, wOL, w1H, w1L, select [4], outH, outL); endmodule // $\operatorname{mux} 32 \times 32$

module $\operatorname{mux} 4(\mathrm{inOH}$, in $0 \mathrm{~L}$, in1 $\mathrm{H}$, in1L, in2H, in2L, in3H, in3L, select, outH, outL);

input in $0 \mathrm{H}$, inOL, in $1 \mathrm{H}$, in $1 \mathrm{~L}$, in $2 \mathrm{H}$, in2L, in $3 \mathrm{H}$, in3L; 


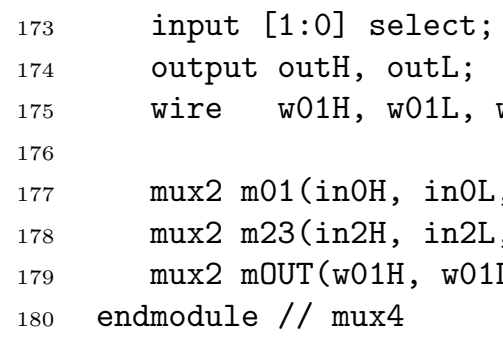

Register Modules

// Asynchronous Processor

// Robert Webb

module Dlatch(DH, DL, En, r, QH, QL);

input DH, DL, En, r;

output QH, QL;

wire enDH,enDL, $\mathrm{Hr}, \mathrm{Lr}, \mathrm{nr}$;

wire notnotQH, notLr;

and \#2 (enDH, DH, En), (enDL, DL, En), (notnotQH, notLr, QH), (Hr, enDH, nr);

not (notLr, Lr), (QL, notnotQH), (nr, r);

or \#1 (QH, Hr, notnotQH), (Lr, r, enDL);

endmodule // Dlatch

module PosTrigger(clk, trig);

input clk;

output trig;

wire notclk;

not \#3 (notclk, clk);

and (trig, clk, notclk);

endmodule // PosTrigger

module reg32(DH, DL, En, clk, r, QH, QL);

input [31:0] DL, DH;

input En, clk, r;

output [31:0] QH, QL;

wire Enr, trig;

PosTrigger $\mathrm{p}(\mathrm{clk}$, trig);

and \#1 (Enr, En, trig);

Dlatch rO(DH[0], DL[0], Enr, r, QH[0], QL[0]);

Dlatch $r 1$ (DH[1], DL[1], Enr, $r, \mathrm{QH}[1], \mathrm{QL}[1])$;

Dlatch r2(DH[2], DL[2], Enr, r, QH[2], QL[2]);

Dlatch r3(DH[3], DL[3], Enr, r, QH[3], QL[3]);

Dlatch r4(DH[4], DL [4], Enr, r, QH[4], QL [4]);

Dlatch r5(DH[5], DL[5], Enr, r, QH[5], QL[5]);

Dlatch r6(DH[6], DL[6], Enr, r, QH[6], QL[6]);

Dlatch r7(DH[7], DL[7], Enr, r, QH[7], QL[7]); 


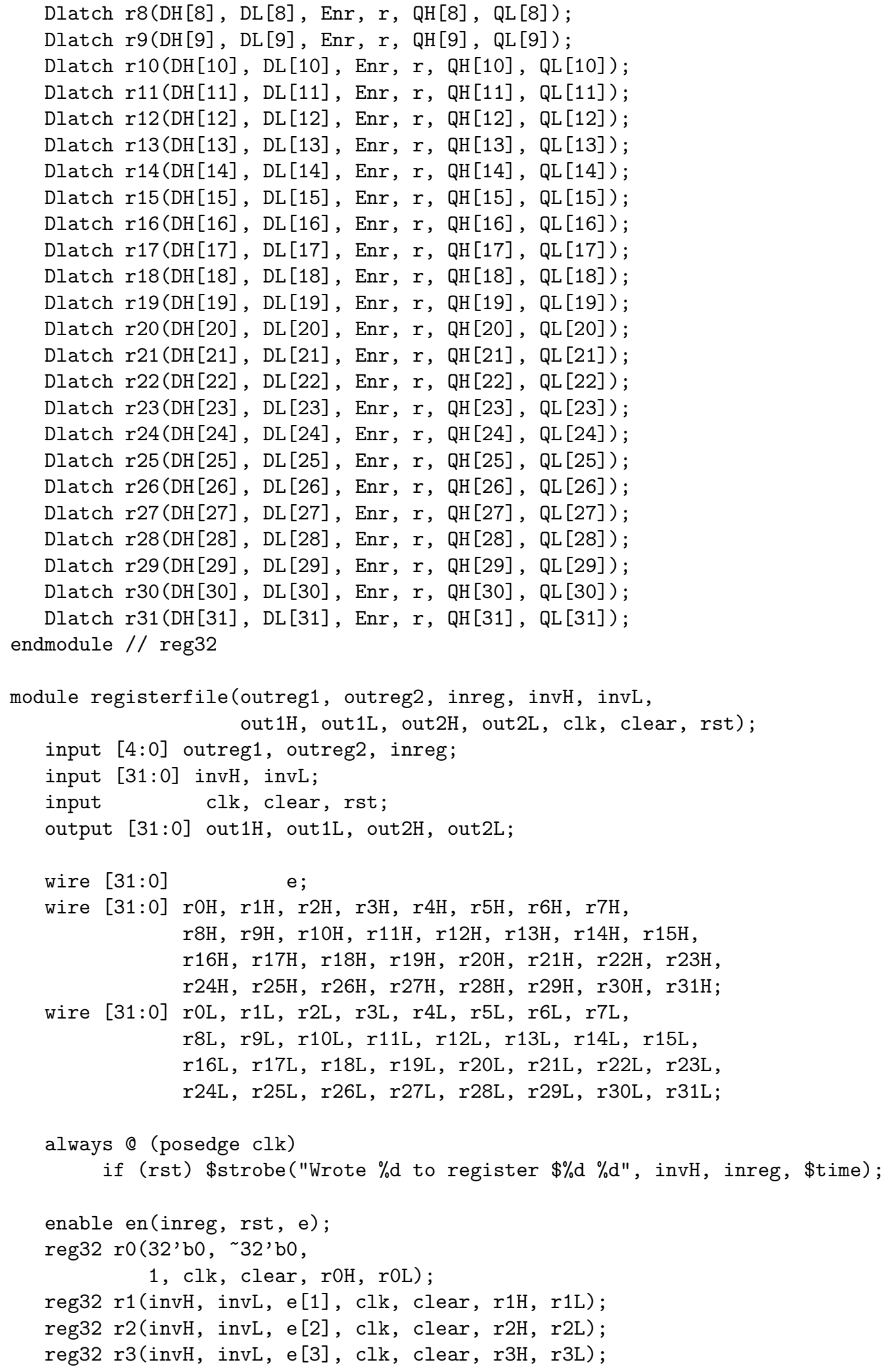


reg32 r4(invH, invL, e[4], clk, clear, r4H, r4L); reg32 r5 (invH, invL, e[5], clk, clear, r5H, r5L); reg32 r6 (invH, invL, e[6], clk, clear, r6H, r6L); reg32 r7 (invH, invL, e[7], clk, clear, r7H, r7L); reg32 r8 (invH, invL, e[8], clk, clear, r8H, r8L); reg32 r9 (invH, invL, e[9], clk, clear, r9H, r9L); reg32 r10 (invH, invL, e[10], clk, clear, r10H, r10L); reg32 r11 (invH, invL, e[11], clk, clear, r11H, r11L); reg32 r12(invH, invL, e[12], clk, clear, r12H, r12L); reg32 r13 (invH, invL, e[13], clk, clear, r13H, r13L); reg32 r14 (invH, invL, e[14], clk, clear, r14H, r14L); reg32 r15 (invH, invL, e[15], clk, clear, r15H, r15L); reg32 r16 (invH, invL, e[16], clk, clear, r16H, r16L); reg32 r17(invH, invL, e[17], clk, clear, r17H, r17L); reg32 r18(invH, invL, e[18], clk, clear, r18H, r18L); reg32 r19 (invH, invL, e[19], clk, clear, r19H, r19L); reg32 r20 (invH, invL, e[20], clk, clear, r20H, r20L); reg32 r21 (invH, invL, e[21], clk, clear, r21H, r21L); reg32 r22(invH, invL, e[22], clk, clear, r22H, r22L); reg32 r23(invH, invL, e[23], clk, clear, r23H, r23L); reg32 r24(invH, invL, e[24], clk, clear, r24H, r24L); reg32 r25 (invH, invL, e[25], clk, clear, r25H, r25L); reg32 r26(invH, invL, e[26], clk, clear, r26H, r26L); reg32 r27 (invH, invL, e[27], clk, clear, r27H, r27L); reg32 r28 (invH, invL, e[28], clk, clear, r28H, r28L); reg32 r29 (invH, invL, e[29], clk, clear, r29H, r29L); reg32 r30 (invH, invL, e[30], clk, clear, r30H, r30L); reg32 r31(invH, invL, e[31], clk, clear, r31H, r31L);

$\operatorname{mux} 32 \times 32 \mathrm{~m} 1(\mathrm{rOH}, \mathrm{rOL}, \mathrm{r} 1 \mathrm{H}, \mathrm{r} 1 \mathrm{~L}, \mathrm{r} 2 \mathrm{H}, \mathrm{r} 2 \mathrm{~L}, \mathrm{r} 3 \mathrm{H}, \mathrm{r} 3 \mathrm{~L}$, $r 4 \mathrm{H}, r 4 \mathrm{~L}, r 5 \mathrm{H}, r 5 \mathrm{~L}, r 6 \mathrm{H}, r 6 \mathrm{~L}, r 7 \mathrm{H}, \mathrm{r} 7 \mathrm{~L}$, r8H, r8L, r9H, r9L, r10H, r10L,r11H, r11L, r12H, r12L, r13H, r13L, r14H, r14L, r15H, r15L, r16H, r16L, r17H, r17L, r18H, r18L, r19H, r19L, r20H, r20L, r21H, r21L, r22H, r22L, r23H, r23L, r24H, r24L, r25H, r25L, r26H, r26L, r27H, r27L, r28H, r28L, r29H, r29L, r30H, r30L, r31H, r31L, outreg1, out1H, out1L);

mux32x32 m2(r0H, r0L, r1H, r1L, r2H, r2L, r3H, r3L, $r 4 \mathrm{H}, r 4 \mathrm{~L}, r 5 \mathrm{H}, r 5 \mathrm{~L}, r 6 \mathrm{H}, \mathrm{r} 6 \mathrm{~L}, \mathrm{r} 7 \mathrm{H}, \mathrm{r} 7 \mathrm{~L}$, $\mathrm{r} 8 \mathrm{H}, \mathrm{r} 8 \mathrm{~L}, \mathrm{r} 9 \mathrm{H}, \mathrm{r} 9 \mathrm{~L}, \mathrm{r} 10 \mathrm{H}, \mathrm{r} 10 \mathrm{~L}, \mathrm{r} 11 \mathrm{H}, \mathrm{r} 11 \mathrm{~L}$, $\mathrm{r} 12 \mathrm{H}, \mathrm{r} 12 \mathrm{~L}, \mathrm{r} 13 \mathrm{H}, \mathrm{r} 13 \mathrm{~L}, \mathrm{r} 14 \mathrm{H}, \mathrm{r} 14 \mathrm{~L}, \mathrm{r} 15 \mathrm{H}, \mathrm{r} 15 \mathrm{~L}$, $\mathrm{r} 16 \mathrm{H}, \mathrm{r} 16 \mathrm{~L}, \mathrm{r} 17 \mathrm{H}, \mathrm{r} 17 \mathrm{~L}, \mathrm{r} 18 \mathrm{H}, \mathrm{r} 18 \mathrm{~L}, \mathrm{r} 19 \mathrm{H}, \mathrm{r} 19 \mathrm{~L}$, $\mathrm{r} 2 \mathrm{OH}, \mathrm{r} 20 \mathrm{~L}, \mathrm{r} 21 \mathrm{H}, \mathrm{r} 21 \mathrm{~L}, \mathrm{r} 22 \mathrm{H}, \mathrm{r} 22 \mathrm{~L}, \mathrm{r} 23 \mathrm{H}, \mathrm{r} 23 \mathrm{~L}$, r24H, r24L, r25H, r25L, r26H, r26L, r27H, r27L, r28H, r28L, r29H, r29L, r30H, r30L, r31H, r31L, outreg2, out $2 \mathrm{H}$, out2L);

endmodule // registerfile

// Instruction Fetch

// Asynchronous Processor 


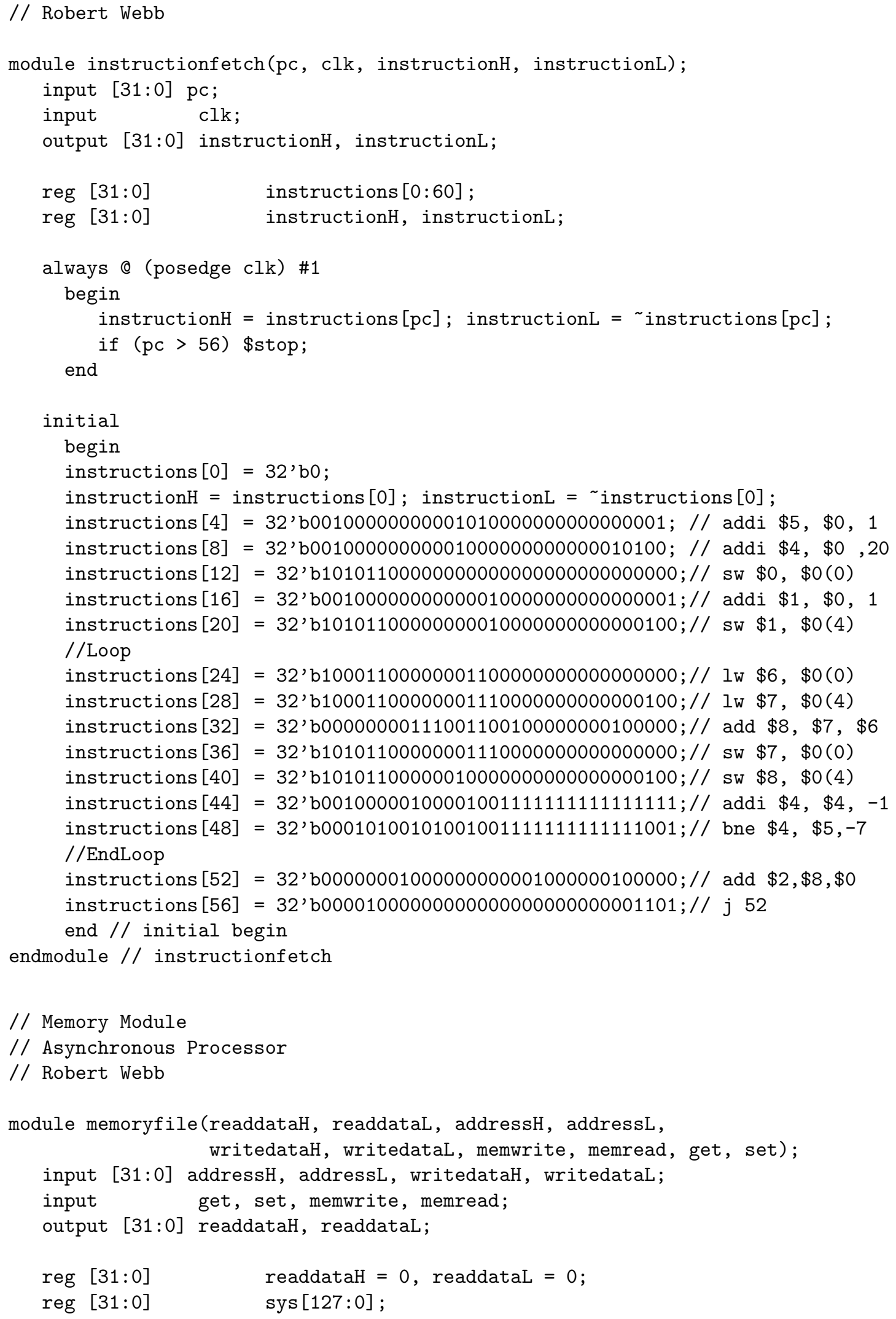




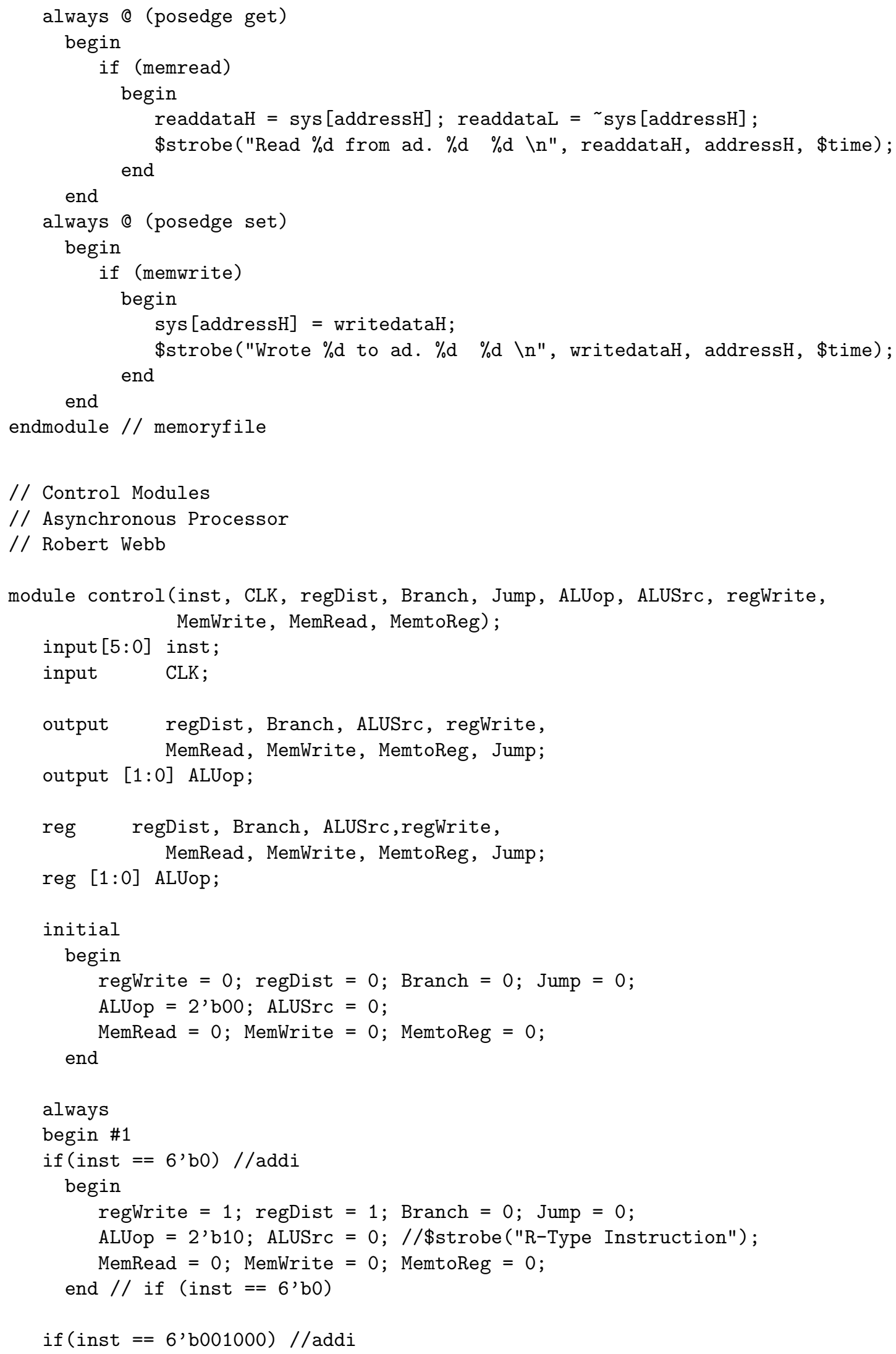




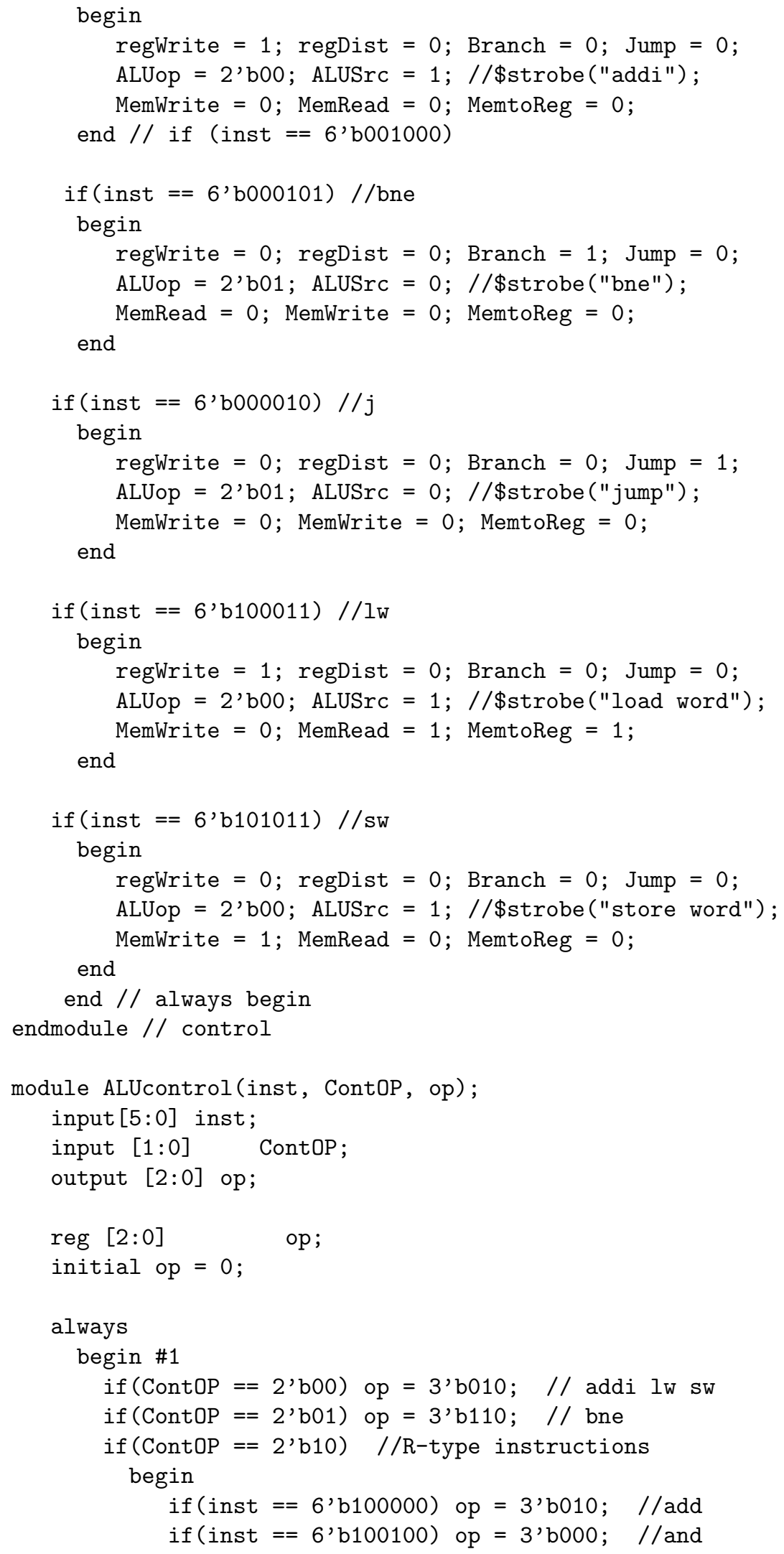




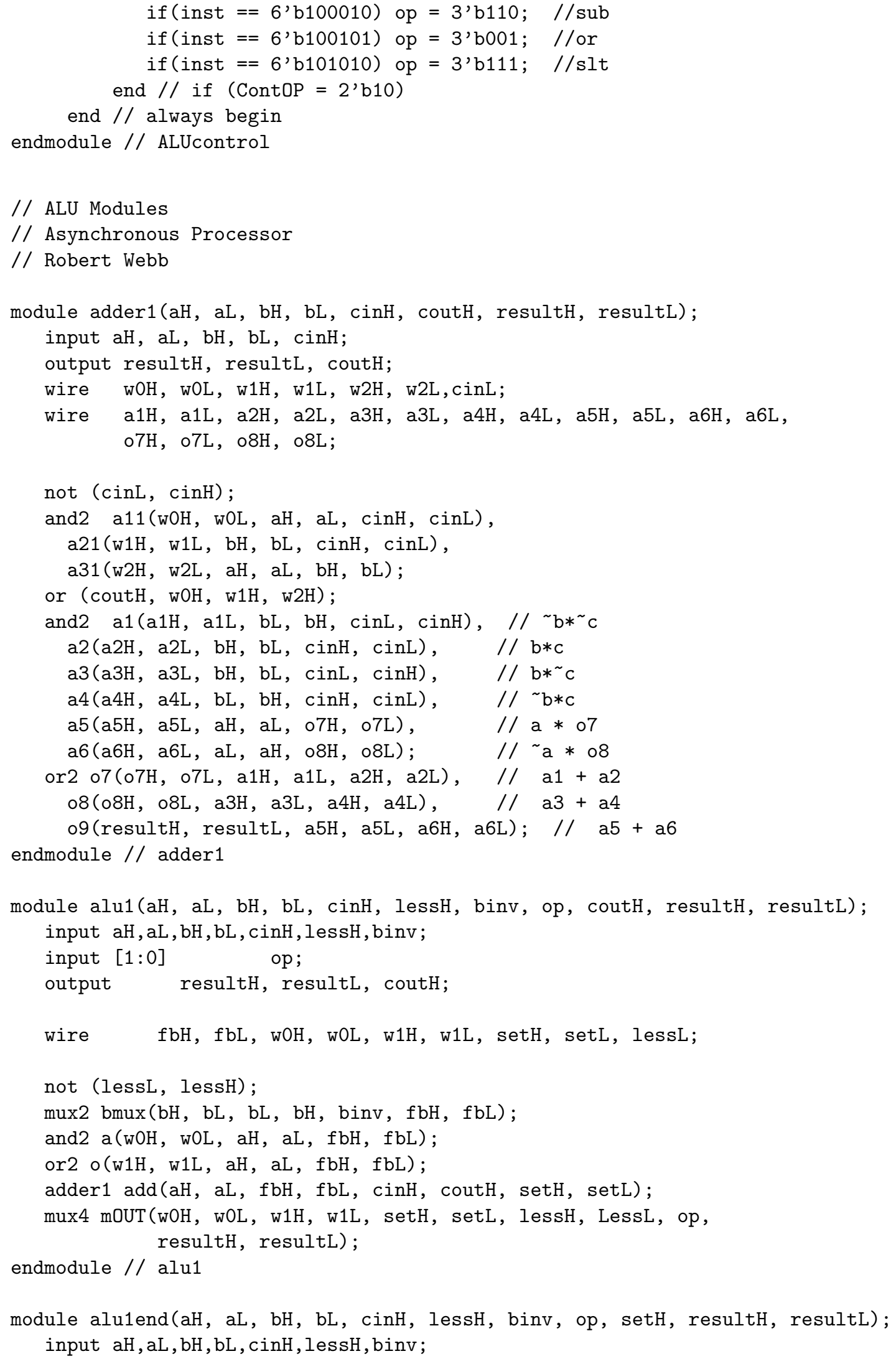




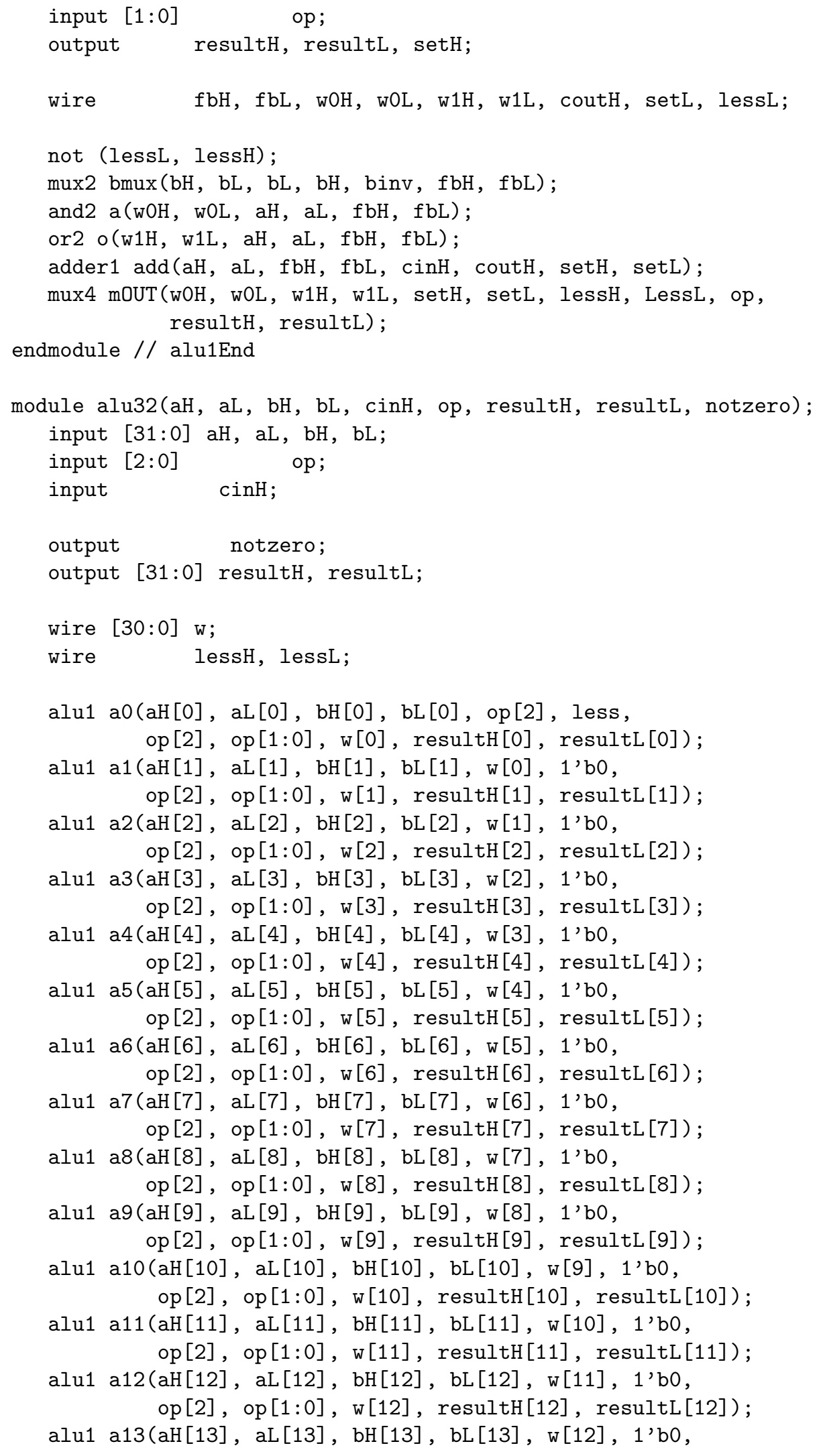




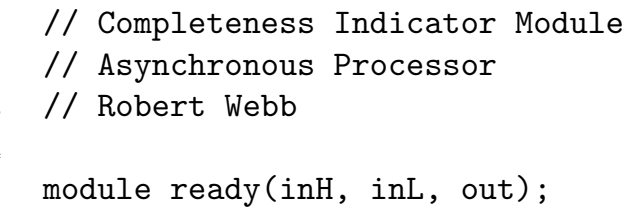

endmodule // alu32

op [2], op [1:0], w[13], resultH[13], resultL[13]); alu1 a14(aH[14], aL[14], bH[14], bL[14], w[13], 1'b0, $\mathrm{op}[2], \mathrm{op}[1: 0], \mathrm{w}[14]$, resultH[14], resultL[14]);

alu1 a15 (aH[15], aL[15], bH[15], bL[15], w[14], 1'b0, op [2], op[1:0], w[15], resultH[15], resultL[15]);

alu1 a16(aH[16], aL[16], bH[16], bL[16], w[15], 1'b0, op [2], op[1:0], w[16], resultH[16], resultL[16]);

alu1 a17 (aH[17], aL[17], bH[17], bL[17], w[16], 1'b0, op [2], op[1:0], w[17], resultH[17], resultL[17]);

alu1 a18 (aH[18], aL[18], bH[18], bL[18], w[17], 1'b0, op [2], op [1:0], w[18], resultH[18], resultL[18]);

alu1 a19(aH[19], aL[19], bH[19], bL[19], w[18], 1'b0, op [2], op[1:0], w[19], resultH[19], resultL[19]);

alu1 a20 (aH[20], aL[20], bH[20], bL[20], w[19], 1'b0, op [2], op[1:0], w[20], resultH[20], resultL[20]);

alu1 a21 (aH[21], aL[21], bH[21], bL[21], w[20], 1'bo, op [2], op[1:0], w[21], resultH[21], resultL[21]);

alu1 a22 (aH [22], aL[22], bH[22], bL[22], w[21], 1'b0, op [2], op[1:0], w[22], resultH[22], resultL[22]);

alu1 a23 (aH[23], aL[23], bH[23], bL[23], w[22], 1'b0, op [2], op[1:0], w[23], resultH[23], resultL[23] );

alu1 a24(aH [24], aL[24], bH[24], bL[24], w[23], 1'b0, op [2], op [1:0], w[24], resultH[24], resultL[24]);

alu1 a25 (aH[25], aL[25], bH[25], bL[25], w[24], 1'b0, op [2], op[1:0], w[25], resultH[25], resultL[25]);

alu1 a26 (aH[26], aL[26], bH[26], bL[26], w[25], 1'bo, op [2], op[1:0], w[26], resultH[26], resultL[26]);

alu1 a27 (aH[27], aL[27], bH[27], bL[27], w[26], 1'b0, op [2], op[1:0], w[27], resultH[27], resultL[27]);

alu1 a28 (aH[28], aL [28], bH[28], bL [28], w[27], 1'b0, op [2], op[1:0], w[28], resultH[28], resultL[28]);

alu1 a29 (aH[29], aL[29], bH[29], bL[29], w[28], 1'b0, op [2], op[1:0], w[29], resultH[29], resultL[29] );

alu1 a30 (aH[30], aL[30], bH[30], bL[30], w[29], 1'b0, op [2], op [1:0], w[30], resultH[30], resultL[30]);

alu1end a31(aH[31], aL[31], bH[30], bL[31], w[30], 1'b0, op[2], op[1:0], less, resultH[31], resultL[031]);

or \#1 (notzero, resultH[31], resultH[30], resultH[29], resultH[28], resultH[27], resultH[26], resultH[25], resultH[24], resultH[23], resultH[22], resultH[21], resultH[20], resultH[19], resultH[18], resultH [17], resultH[16], resultH[15], resultH [14], resultH [13], resultH[12], resultH[11], resultH[10], resultH[9], resultH[8], resultH[7], resultH[6], resultH[5], resultH[4], resultH[3], resultH[2], resultH[1], resultH[0]); 


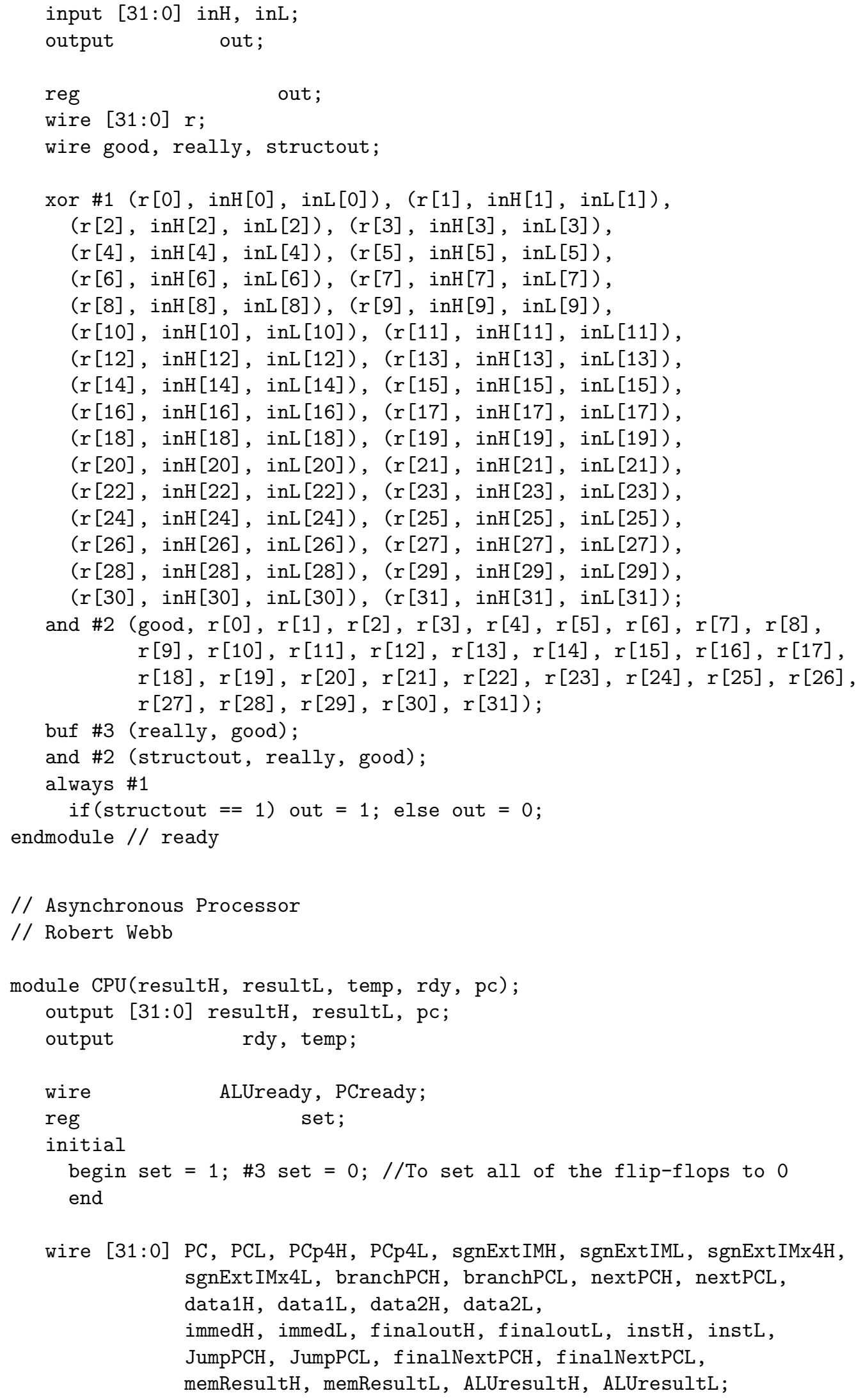




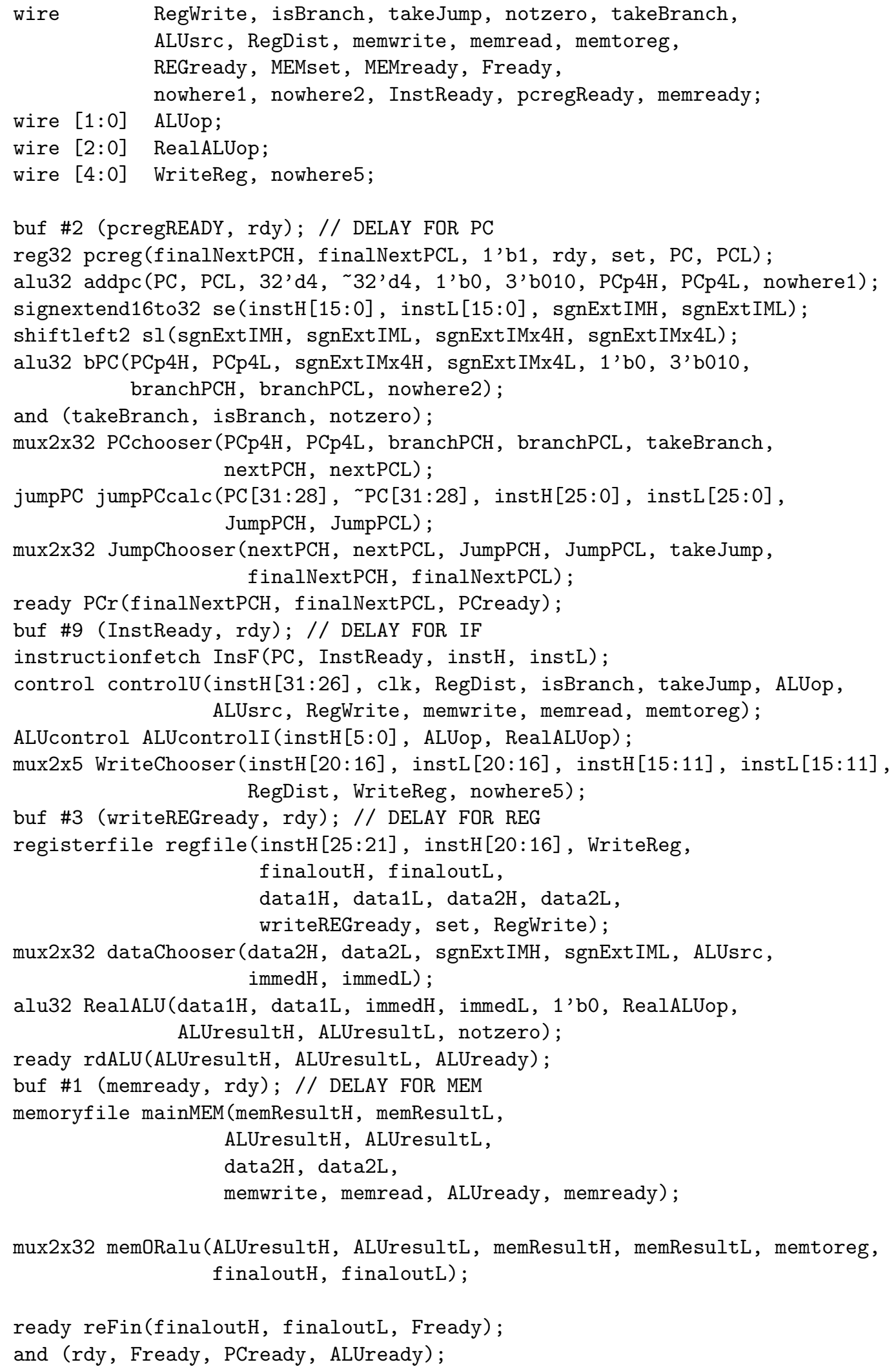




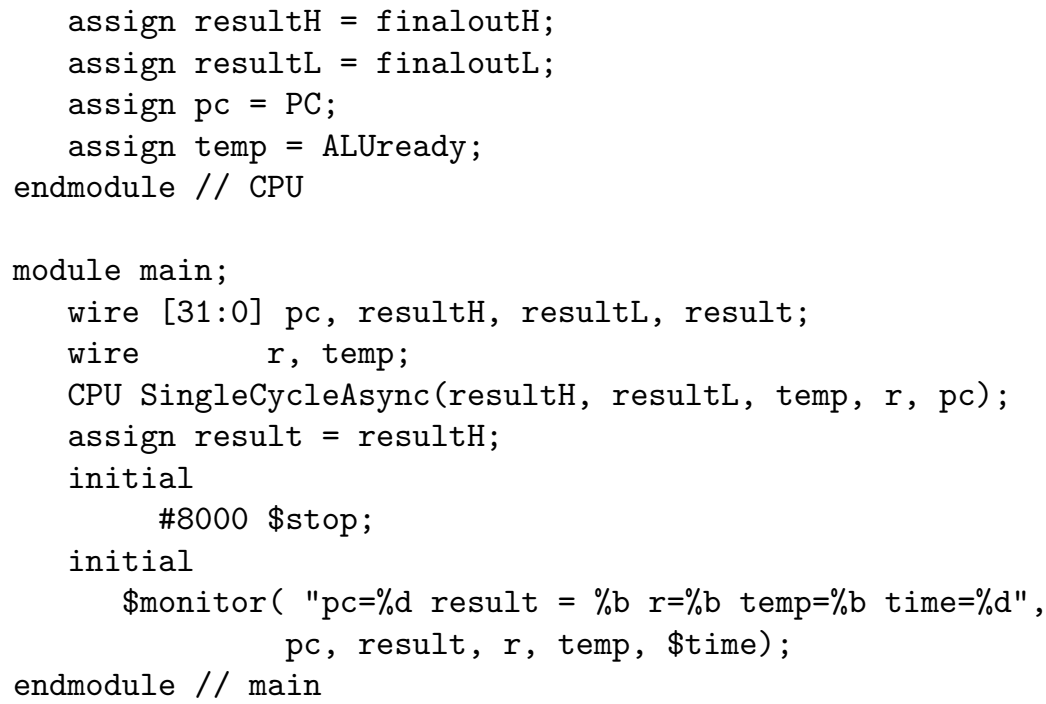




\section{Appendix $\mathrm{C}$}

\section{The Complete Pipelined}

\section{Asynchronous Source Code}

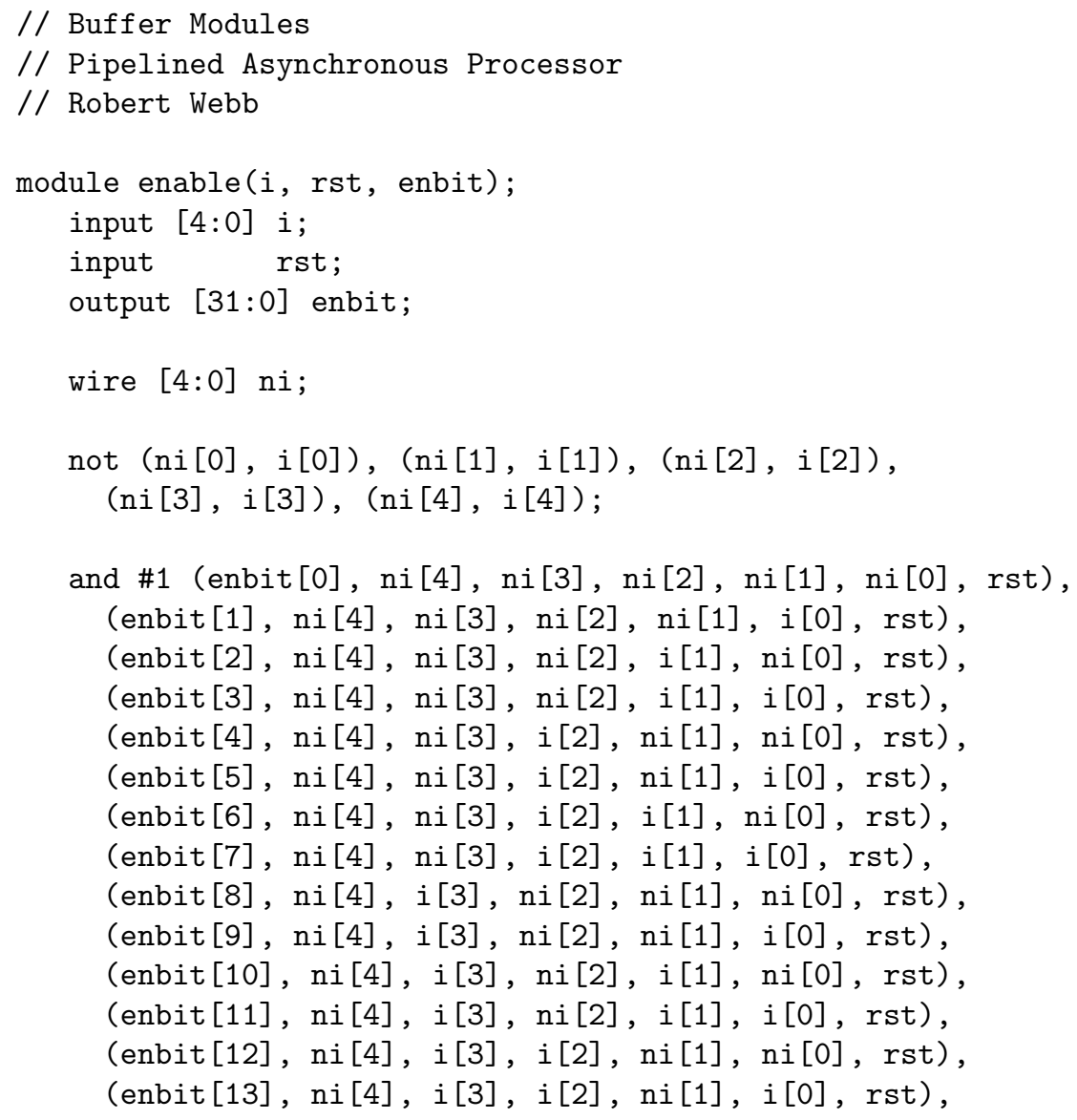




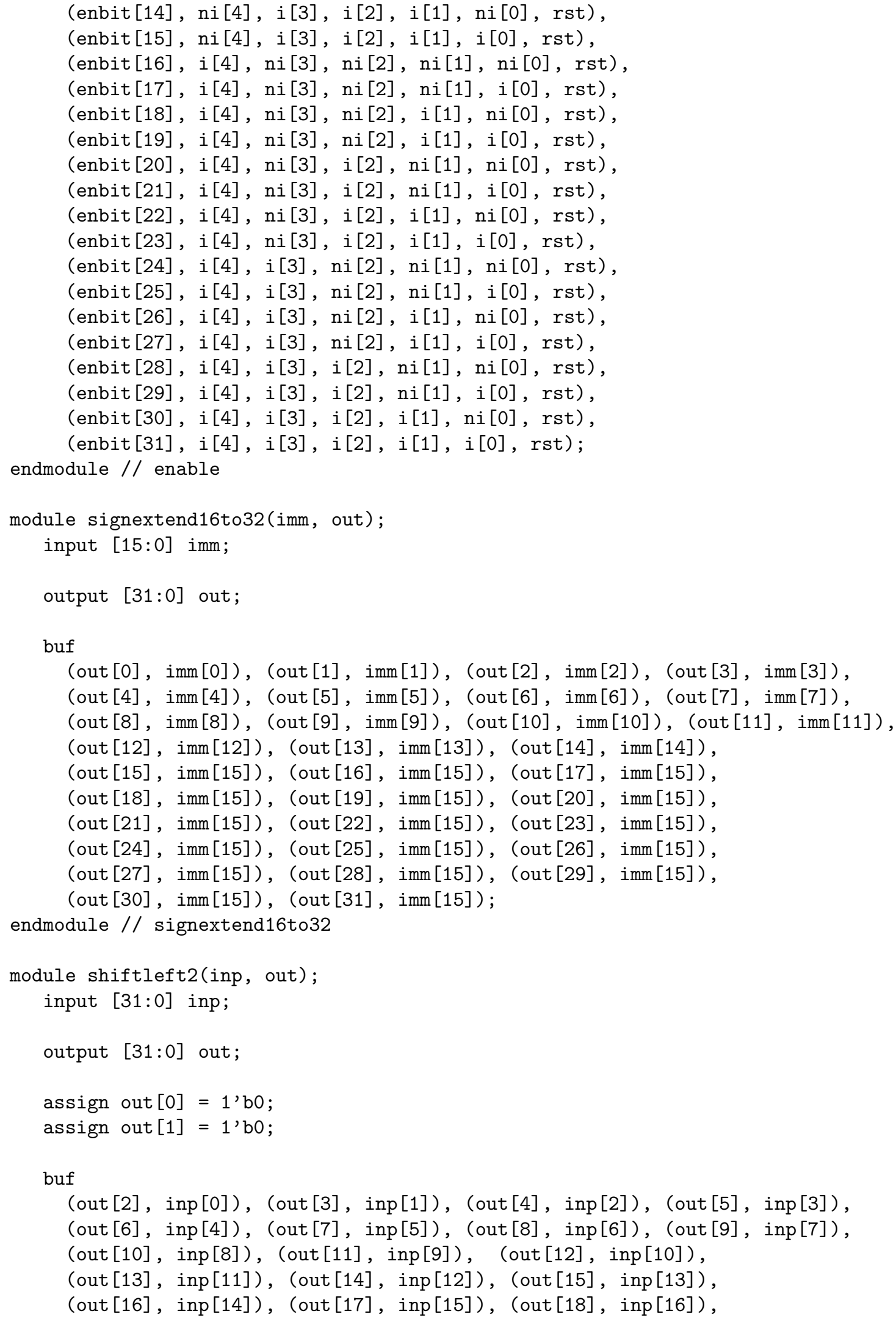




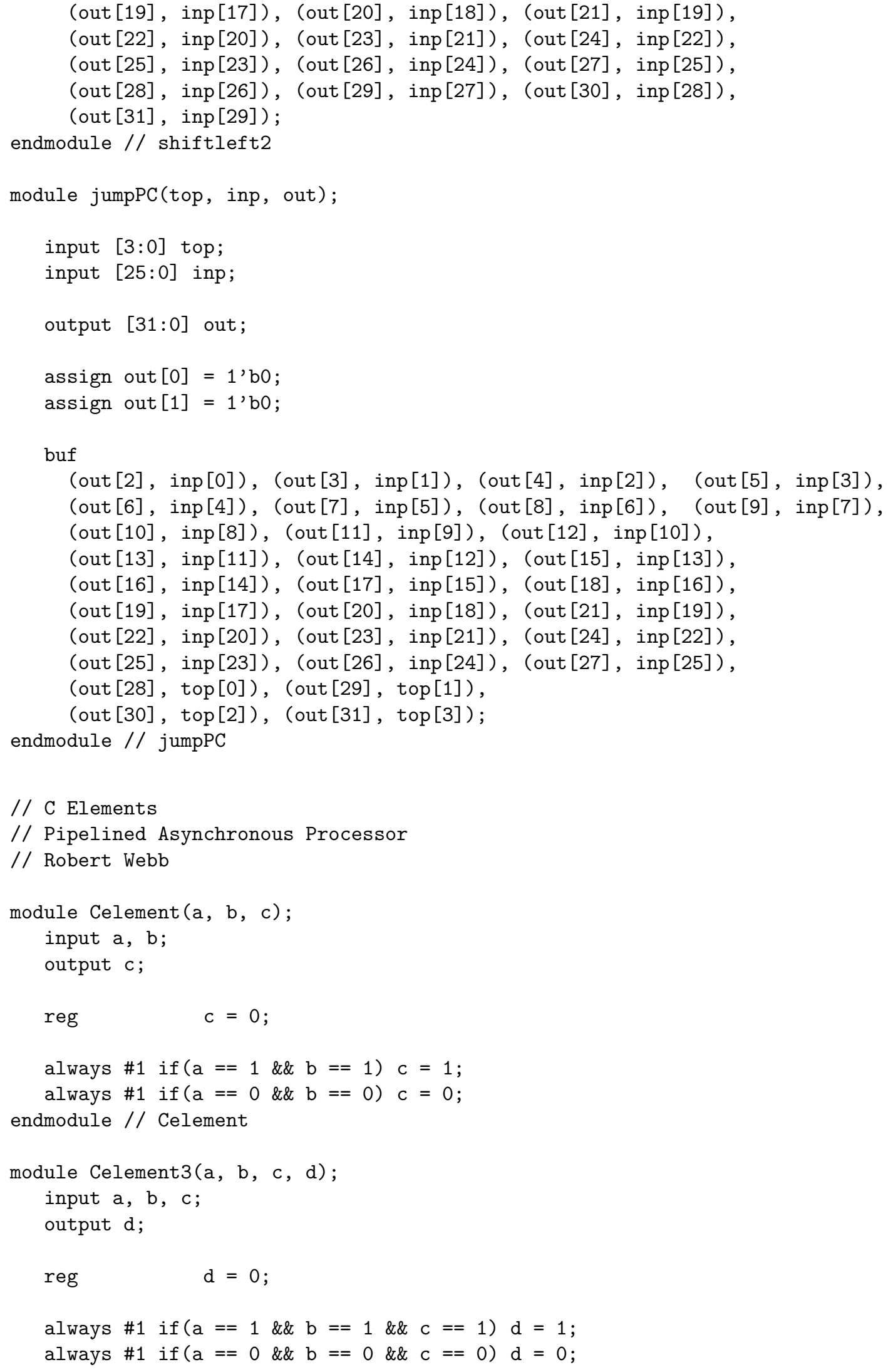




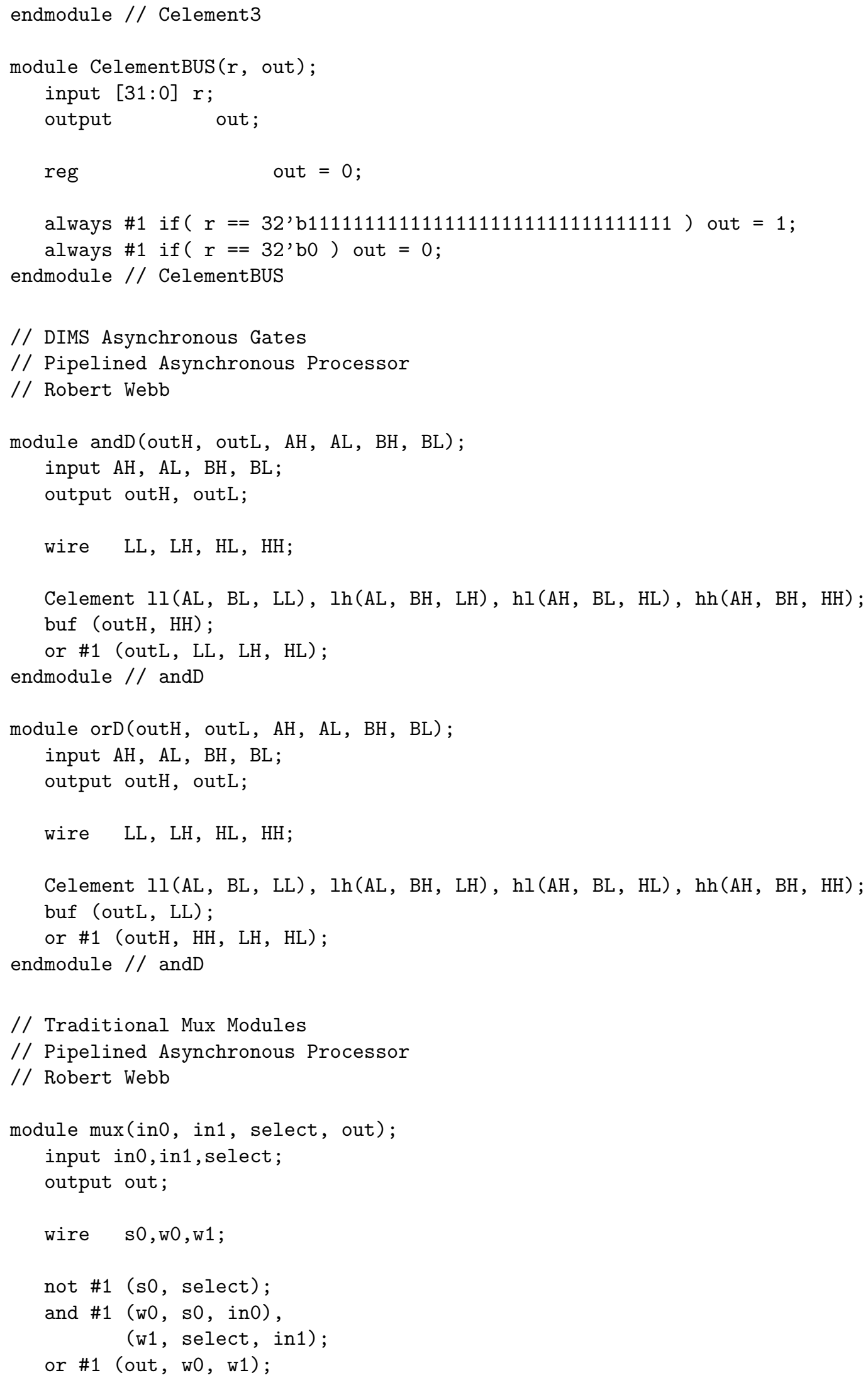




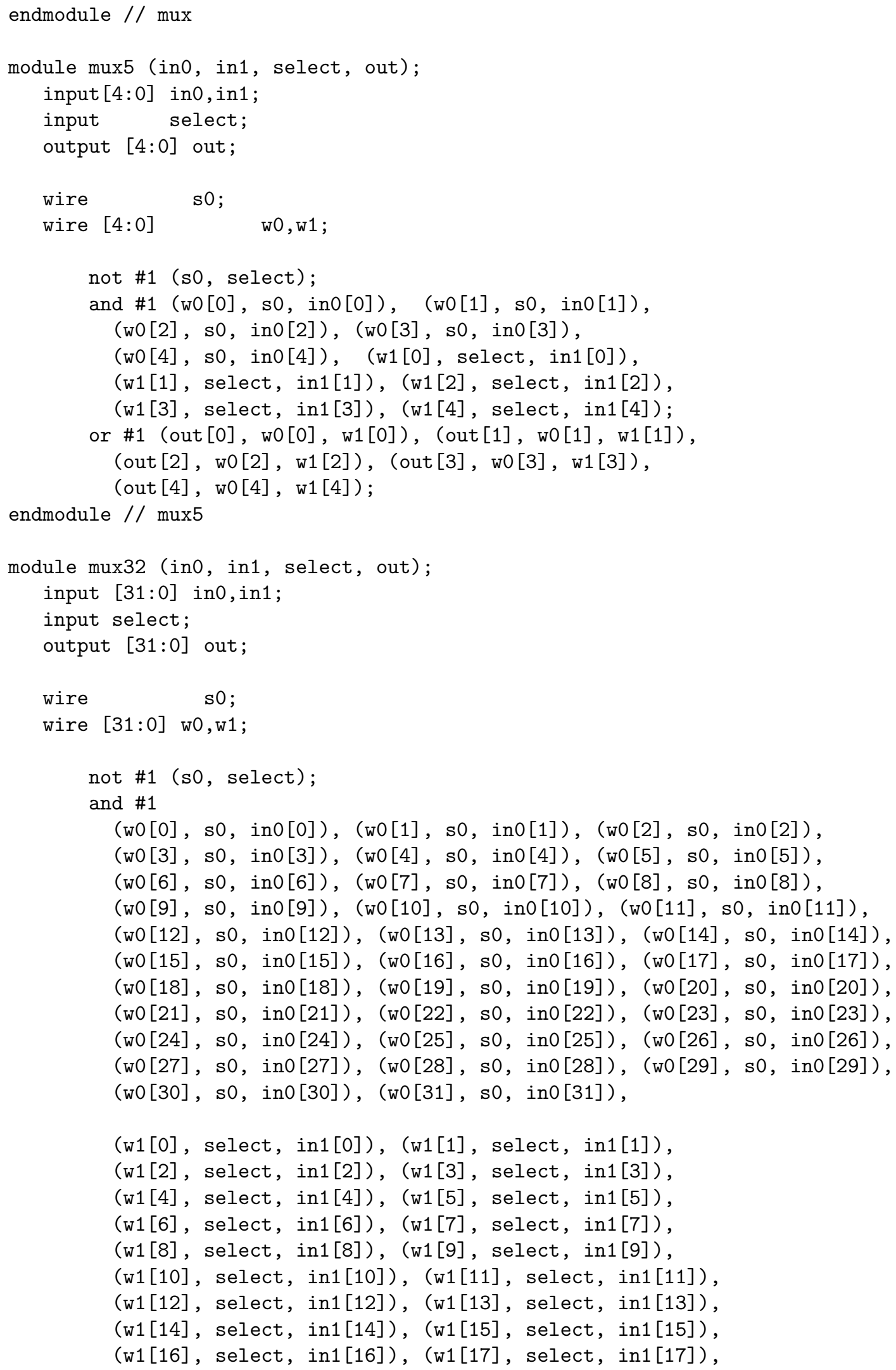




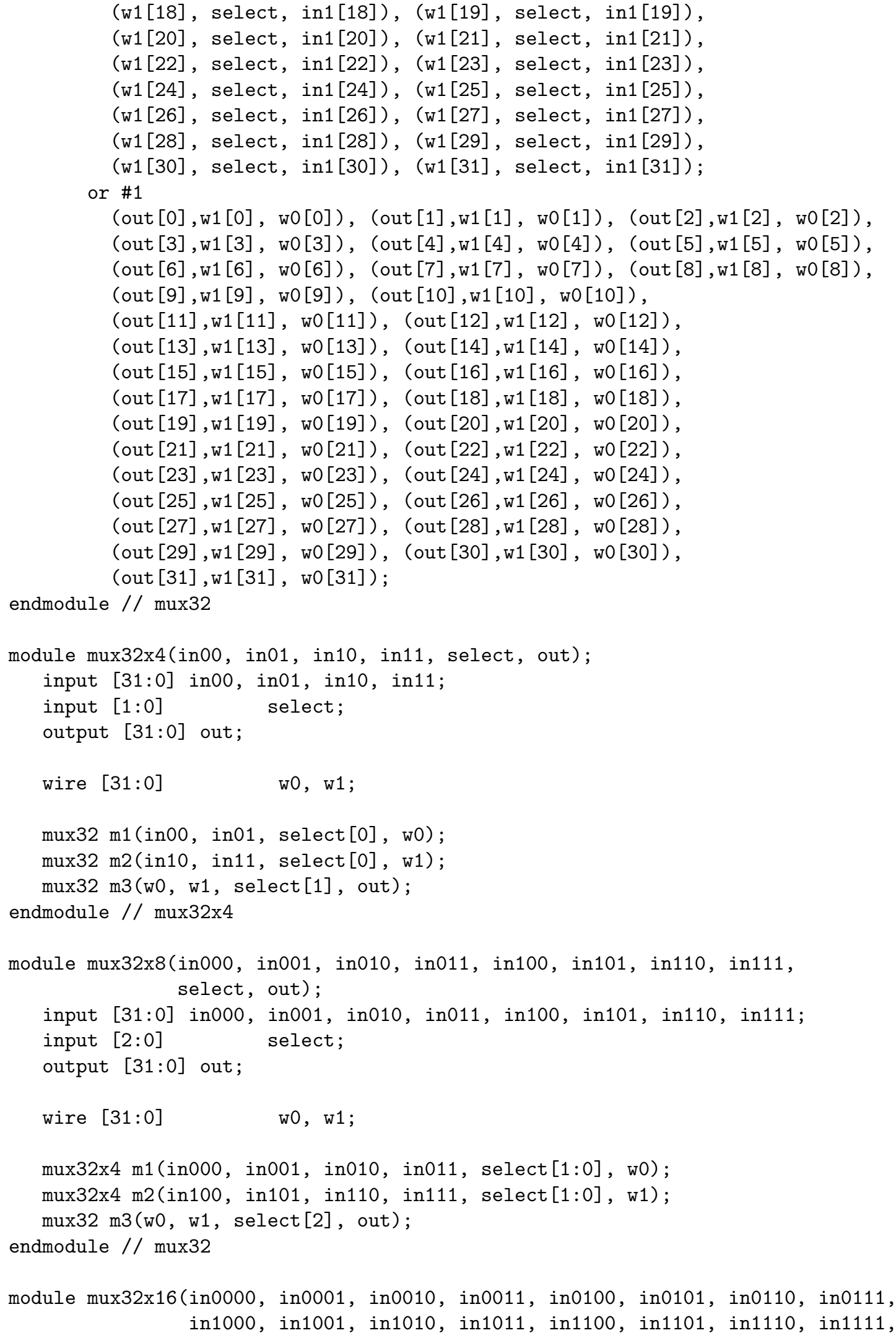




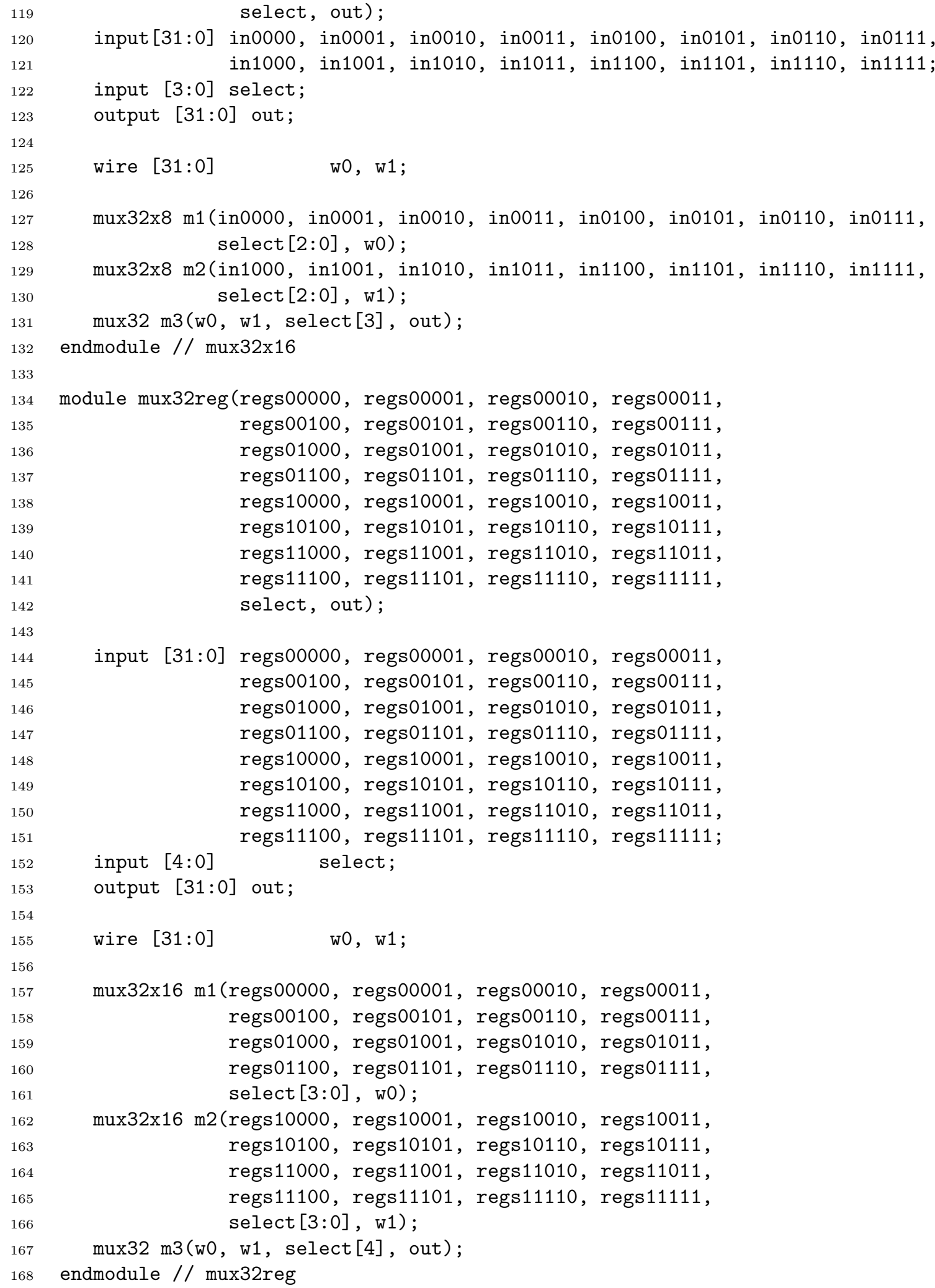




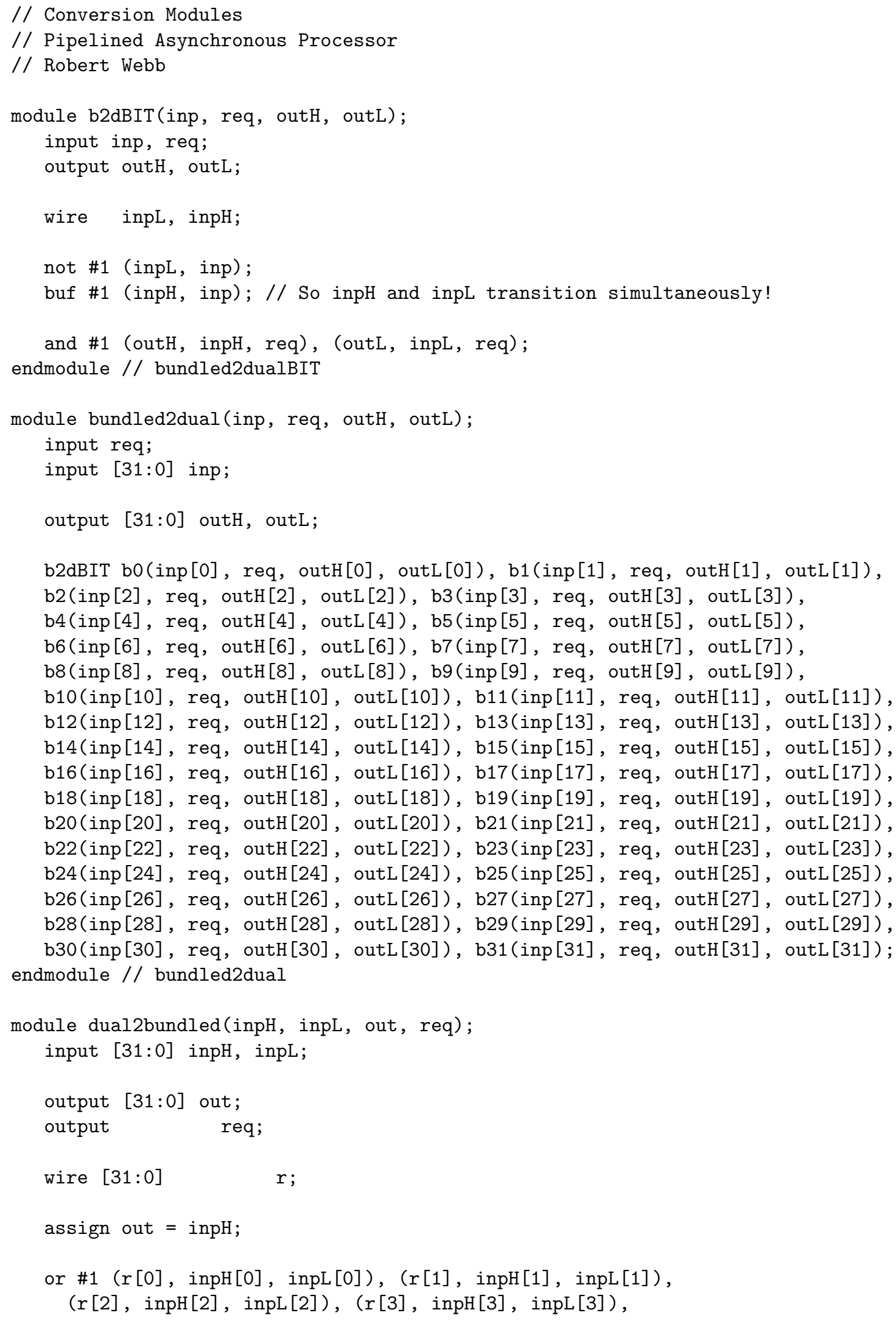




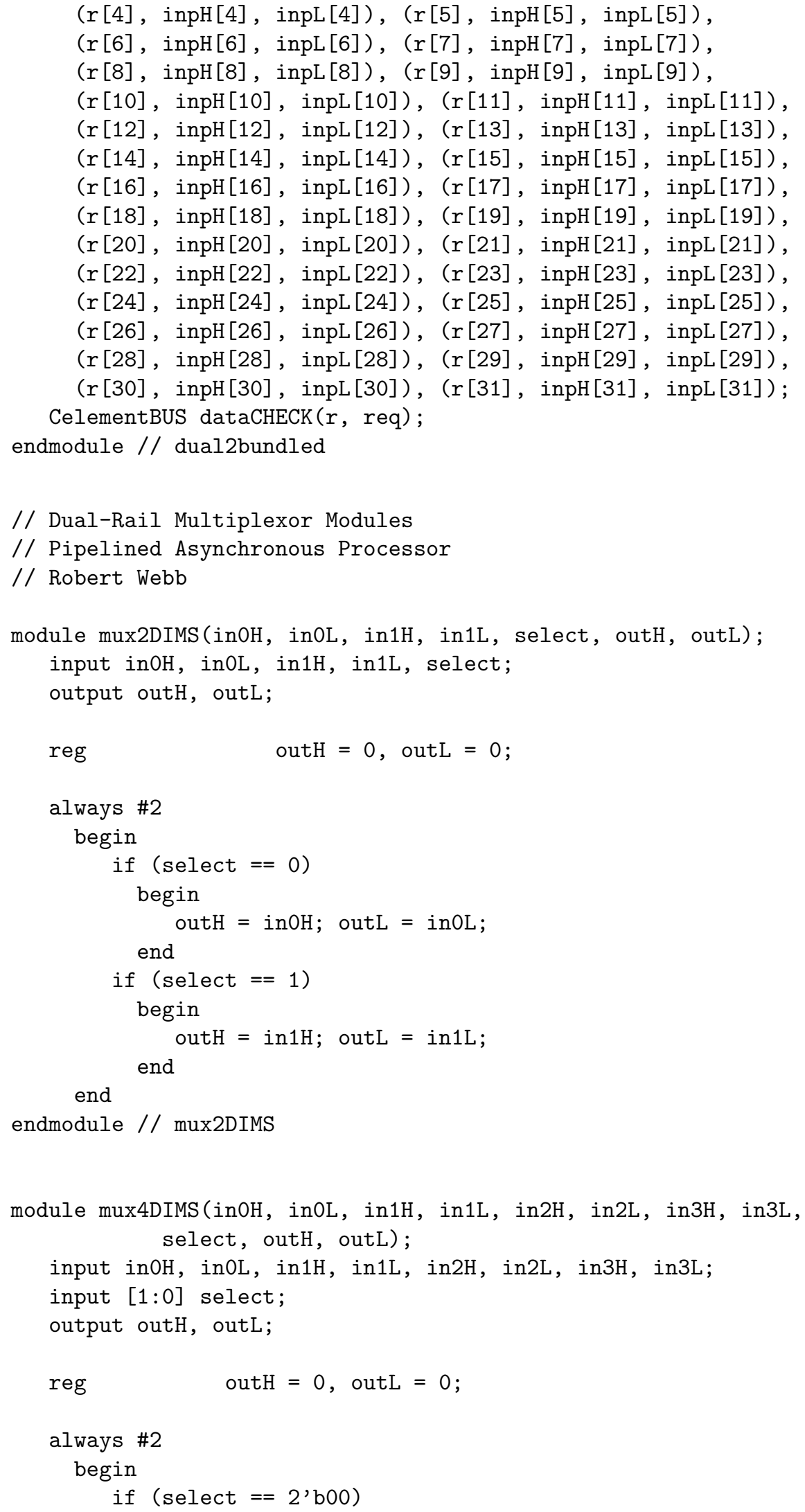




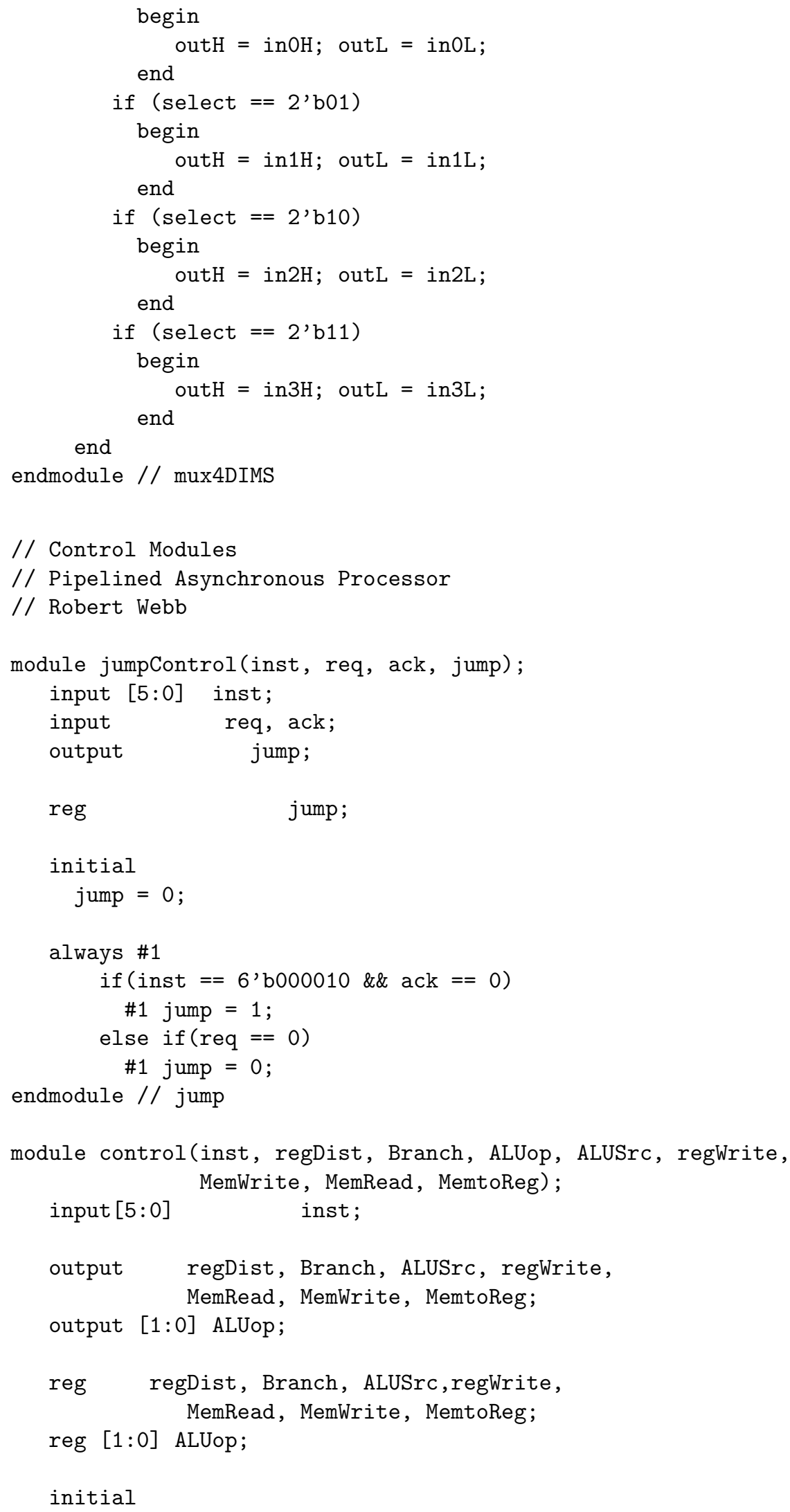




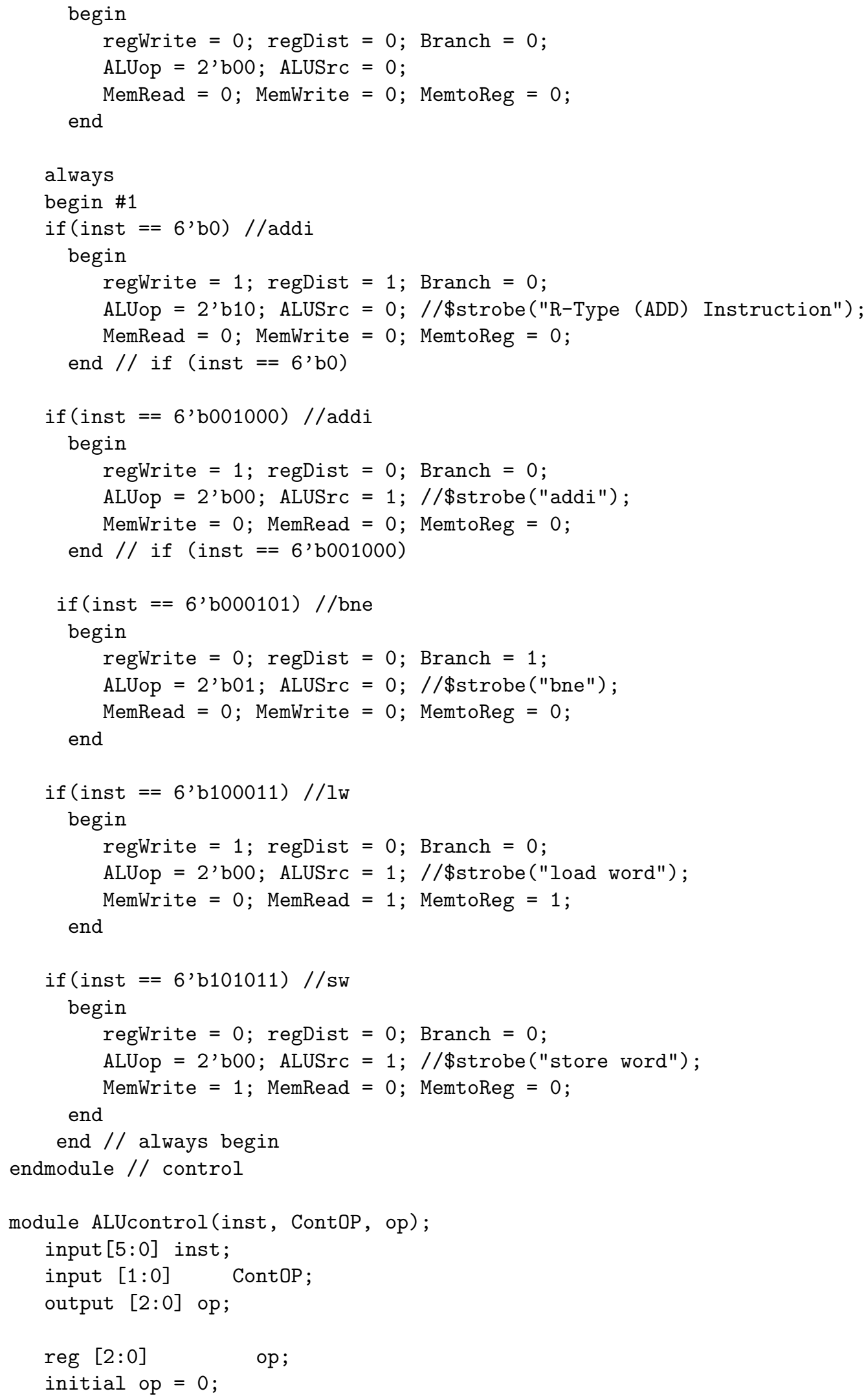




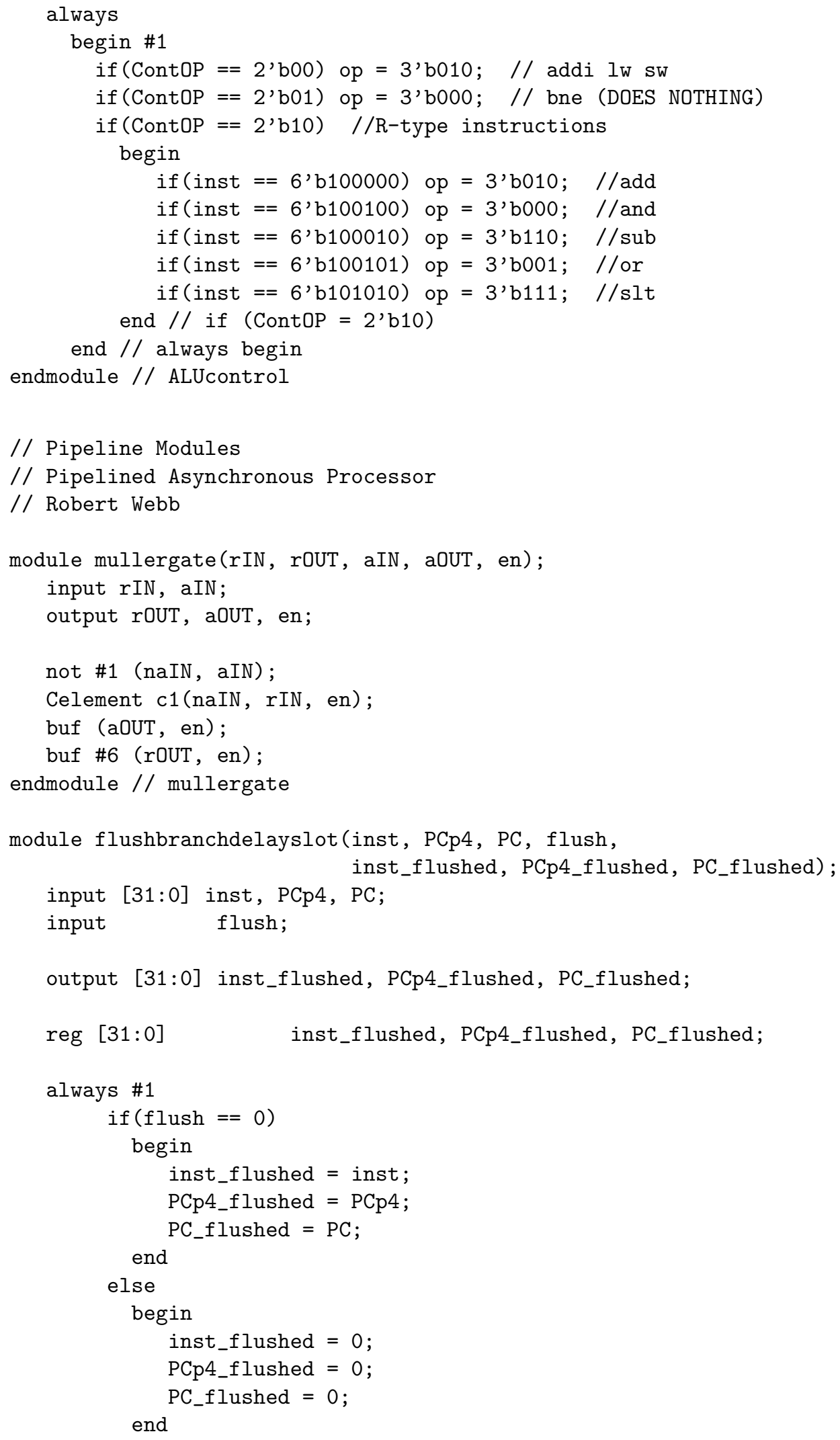




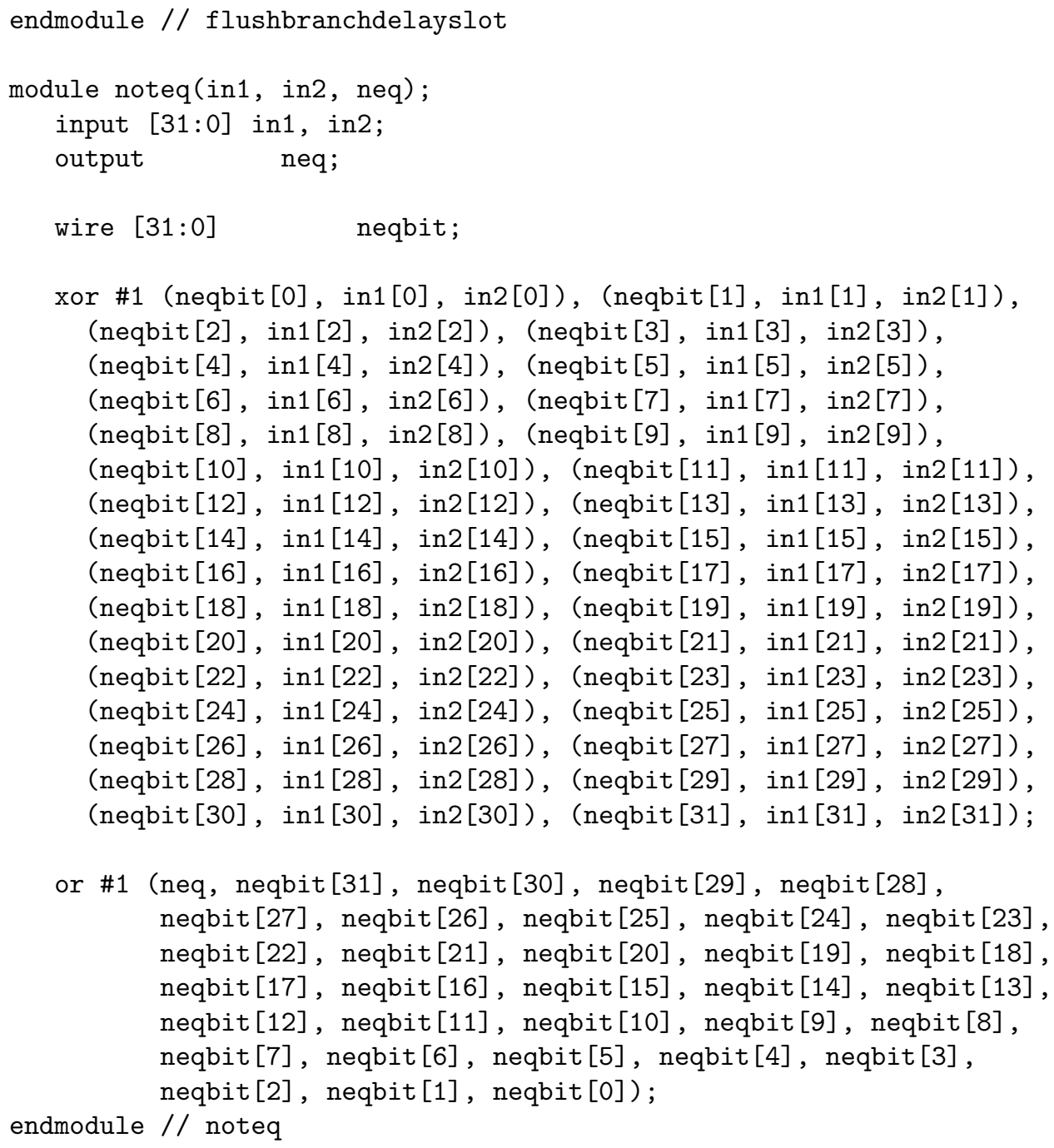




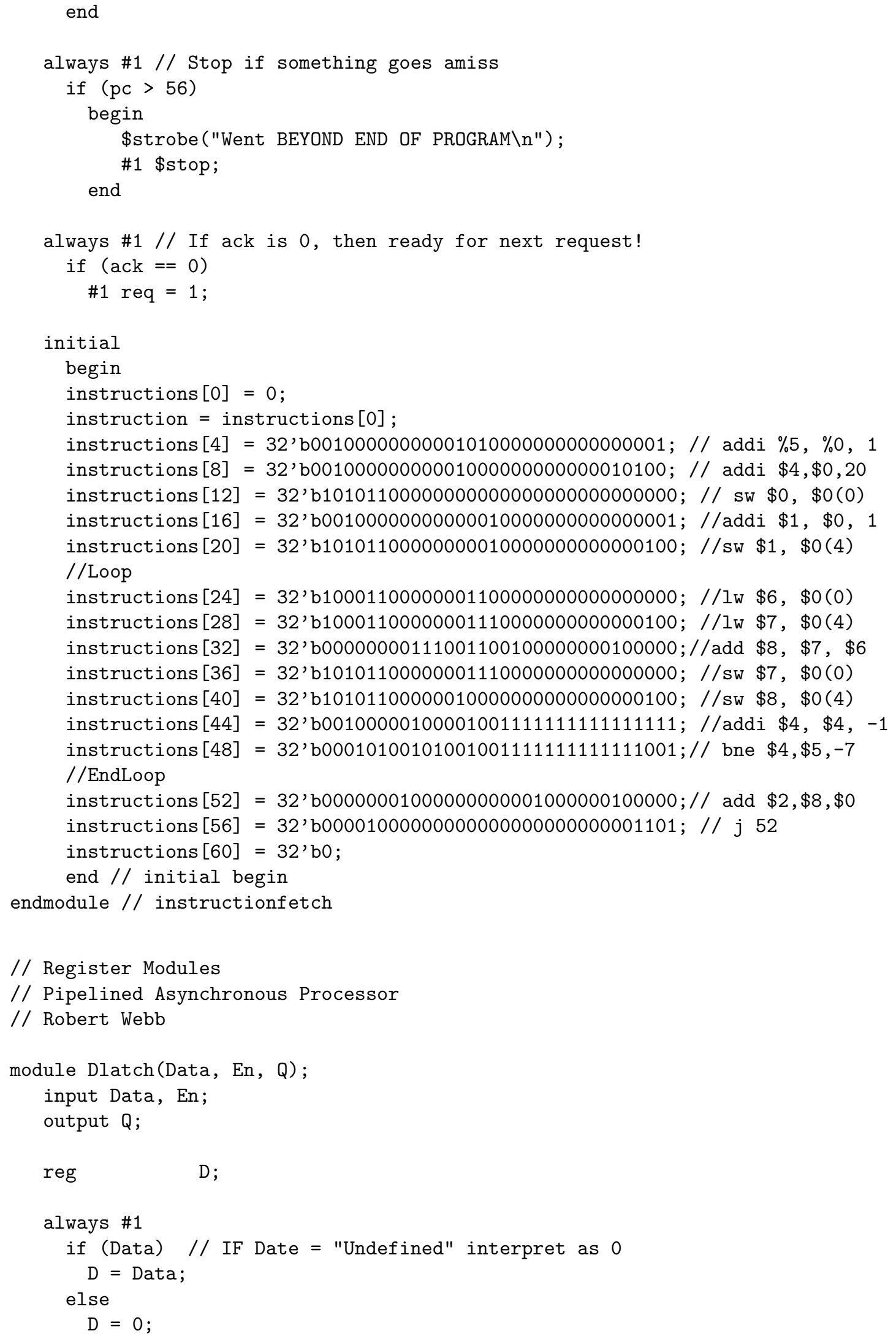




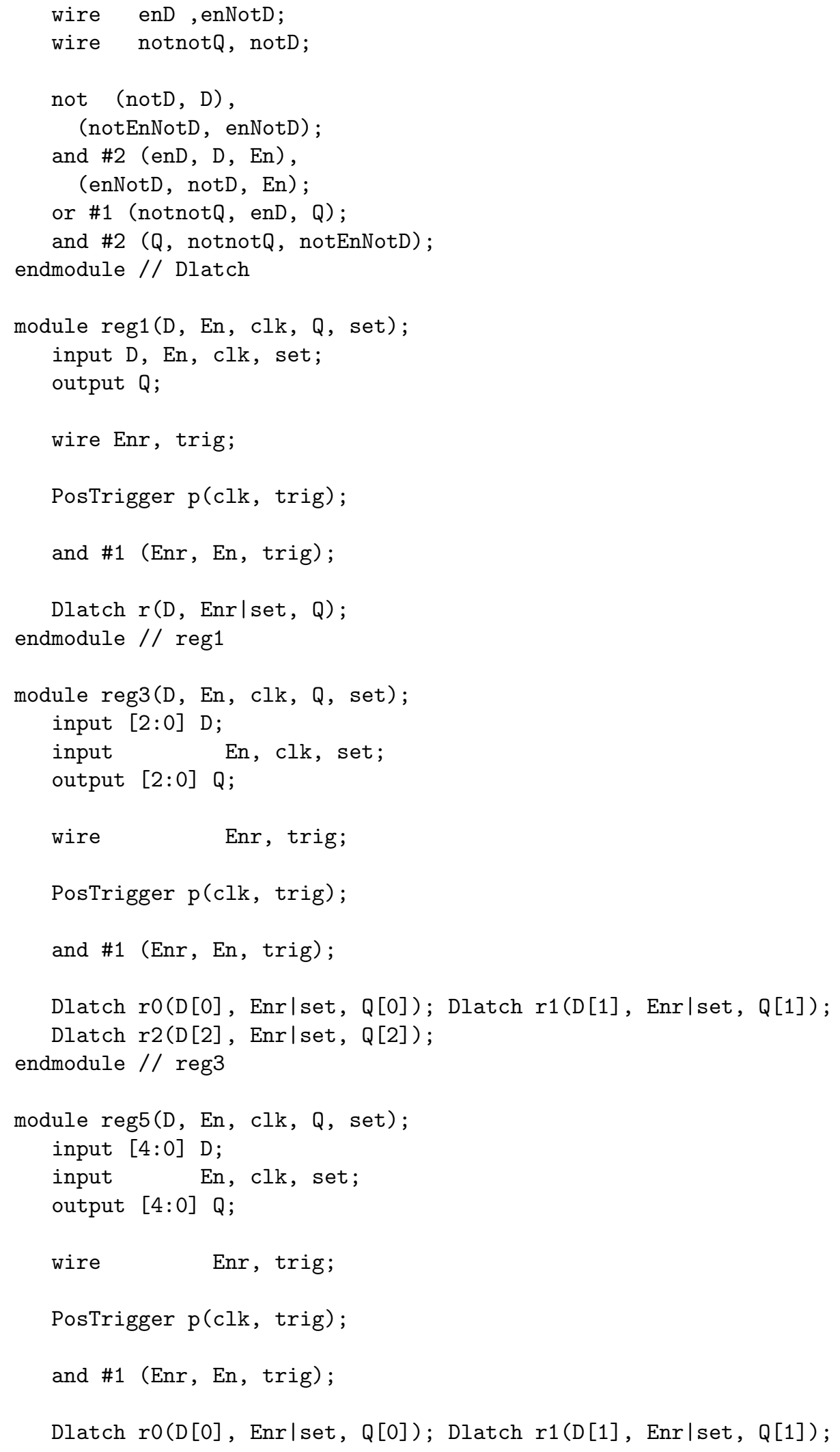




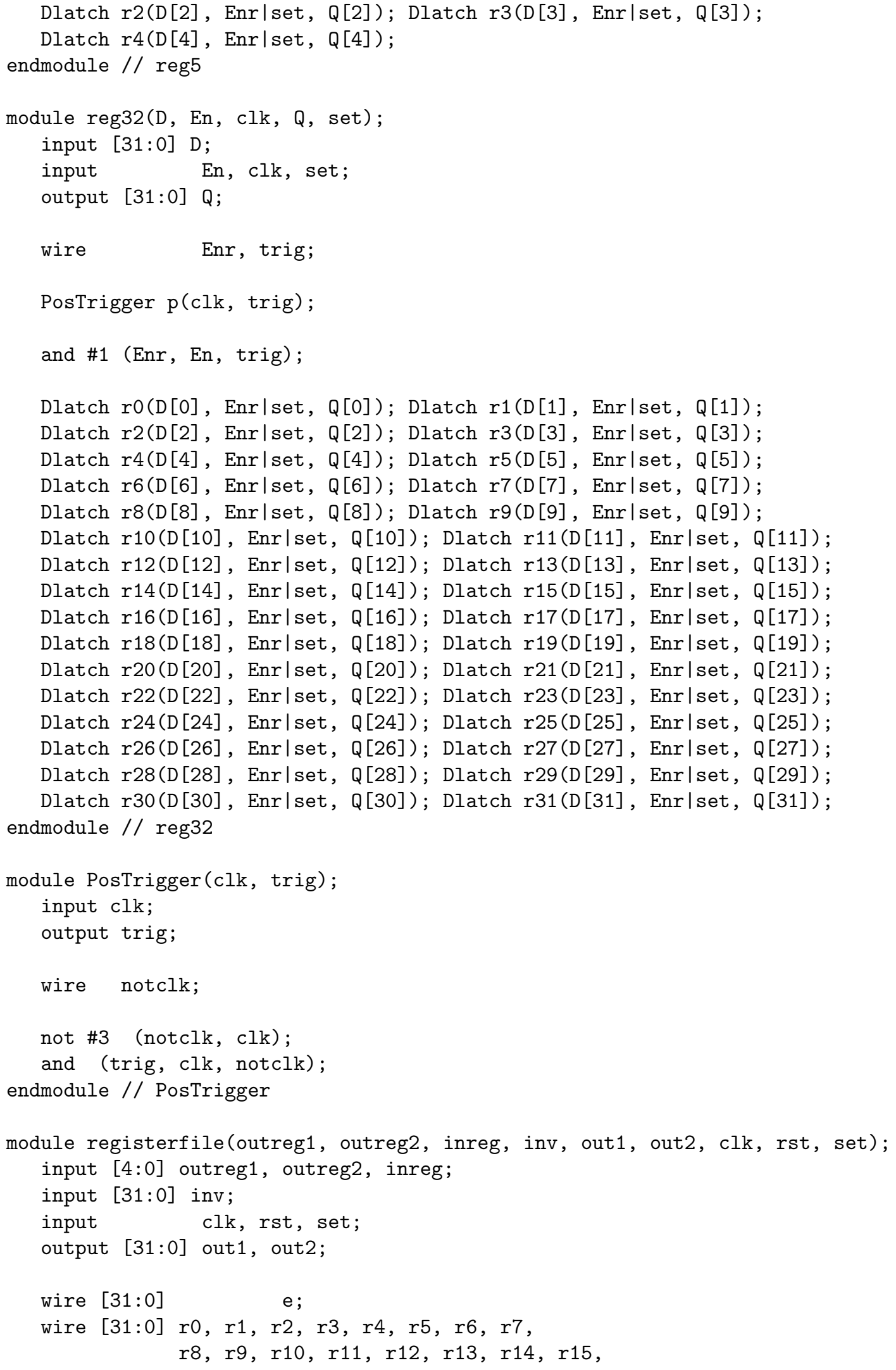




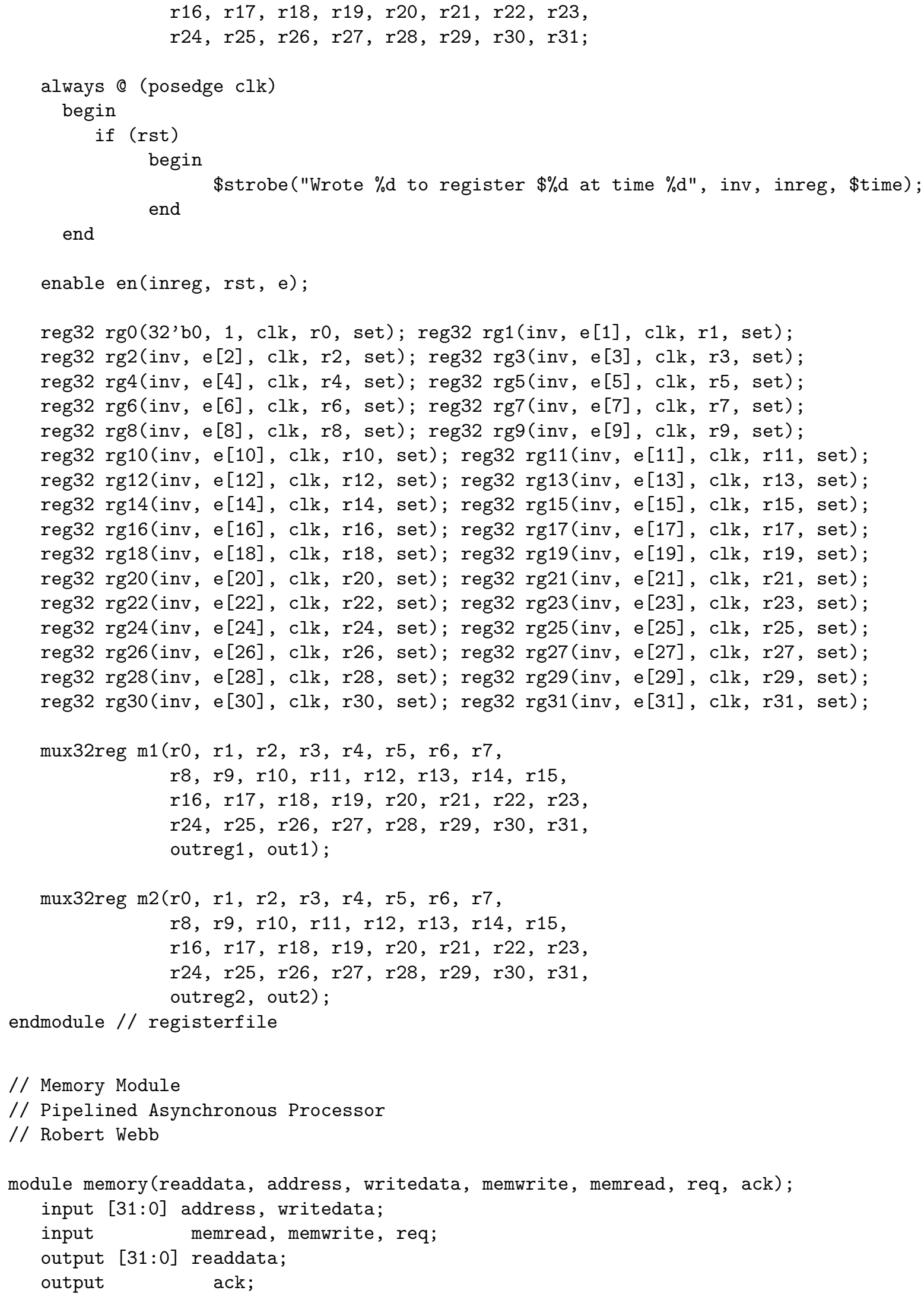




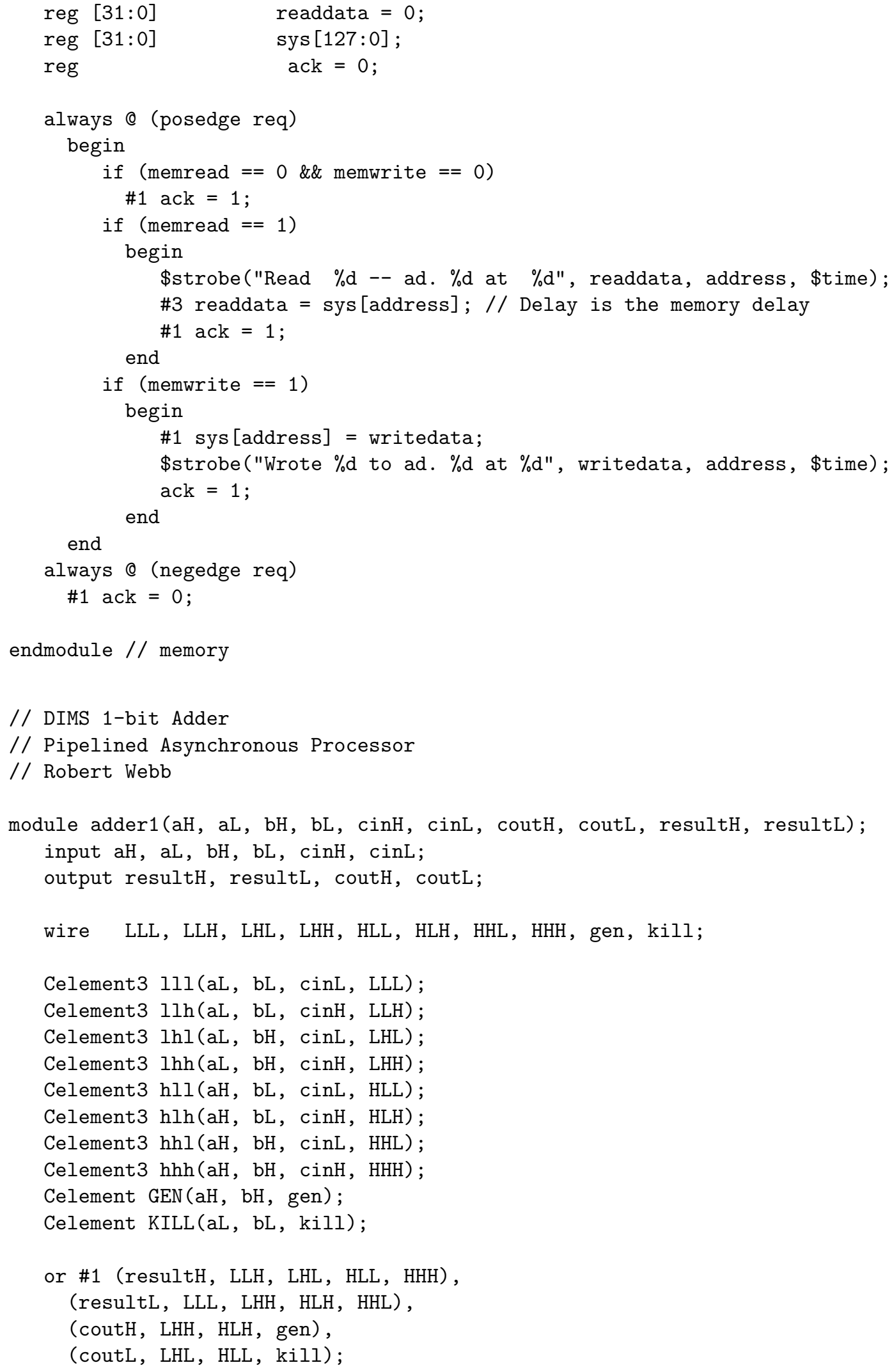


endmodule // adder 1

// ALU Modules

// Pipelined Asynchronous Processor

// Robert Webb coutH, coutL, resultH, resultL);

input $\mathrm{aH}, \mathrm{aL}, \mathrm{bH}, \mathrm{bL}, \mathrm{cinH}, \mathrm{cinL}$, lessH, lessL, binv;

input $[1: 0] \quad o p$;

output resultH, resultL, coutH, coutL;

wire $\quad \mathrm{fbH}, \mathrm{fbL}, \mathrm{wOH}, \mathrm{wOL}, \mathrm{w} 1 \mathrm{H}, \mathrm{w} 1 \mathrm{~L}$, setH, setL;

$\operatorname{mux} 2 \mathrm{DIMS} \operatorname{bmux}(b H, b L, b L, b H, b i n v, f b H, f b L)$;

andD a(wOH, wOL, aH, aL, fbH, fbL);

orD o(w1H, w1L, aH, aL, fbH, fbL);

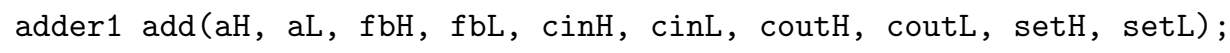

mux4DIMS mOUT(wOH, wOL, w1H, w1L, setH, setL, lessH, LessL, op, resultH, resultL);

endmodule // alu1

module alulend $(\mathrm{aH}, \mathrm{aL}, \mathrm{bH}, \mathrm{bL}, \mathrm{cinH}, \mathrm{cinL}, \mathrm{essH}, \mathrm{lessL}, \mathrm{binv}, \mathrm{op}$, setH, setL, resultH, resultL);

input $\mathrm{aH}, \mathrm{aL}, \mathrm{bH}, \mathrm{bL}, \mathrm{cinH}$, cinL, lessH, lessL, binv;

input $[1: 0] \quad$ op;

output resultH, resultL, setH, setL;

wire $\mathrm{fbH}, \mathrm{fbL}, \mathrm{wOH}, \mathrm{wOL}, \mathrm{w} 1 \mathrm{H}, \mathrm{w} 1 \mathrm{~L}$, coutH, coutL, setL, lessL;

$\operatorname{mux} 2 \mathrm{DIMS} \operatorname{bmux}(b H, b L, b L, b H, b i n v, f b H, f b L)$;

andD a(wOH, wOL, aH, aL, fbH, fbL);

orD o(w1H, w1L, aH, aL, fbH, fbL);

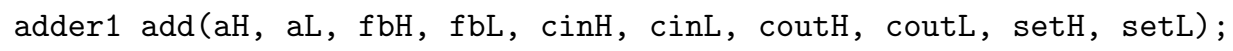

mux4DIMS mOUT(wOH, wOL, w1H, w1L, setH, setL, lessH, LessL, op, resultH, resultL);

endmodule // alu1End

module alu32(aH, aL, bH, bL, op, resultH, resultL, notzero);

input [31:0] aH, aL, bH, bL;

input $[2: 0] \quad$ op;

output notzero;

output [31:0] resultH, resultL;

wire [30:0] $\mathrm{wH}, \mathrm{wL}$;

wire lessH, lessL, aReady, bReady, inpReady, nOp2, cinH, cinL;

xor \#1 (aReady, aH[0], aL[0]);

xor \#1 (bReady, bH[0], bL[0]);

and \#2 (inpReady, aReady, bReady); 
not \#1 (nOp2, op [2]);

and \#2 (cinH, op [2], inpReady);

and \#2 (cinL, nOp2, inpReady);

alu1 a0(aH[0], aL[0], bH[0], bL[0], cinH, cinL, lessH, lessL, op [2], op [1:0], $\mathrm{wH}[0], w L[0]$, resultH[0], resultL[0]);

alu1 a1 (aH[1], aL[1], bH[1], bL[1], wH[0], wL[0], 0, 1, op [2], op[1:0], wH[1], wL[1], resultH[1], resultL[1]);

alu1 a2 (aH[2], aL[2], bH[2], bL[2], wH[1], wL[1], 0, 1, op [2], op [1:0], wH[2], wL[2], resultH[2], resultL[2]);

alu1 a3(aH[3], aL[3], bH[3], bL[3], wH[2], wL[2], 0, 1, op [2], op [1:0], wH[3], wL[3], resultH[3], resultL[3]);

alu1 a4 (aH[4], aL[4], bH[4], bL[4], wH[3], wL[3], 0, 1, op [2], op[1:0], wH[4], wL[4], resultH[4], resultL[4]);

alu1 a5 (aH[5], aL[5], bH[5], bL[5], wH[4], wL[4], 0, 1, op [2], op[1:0], wH[5], wL[5], resultH[5], resultL[5]);

alu1 a6(aH[6], aL[6], bH[6], bL[6], wH[5], wL[5], 0, 1 , op[2], op[1:0], wH[6], wL[6], resultH[6], resultL[6]);

alu1 a7 (aH[7], aL[7], bH[7], bL[7], wH[6], wL[6], 0, 1, op [2], op[1:0], $\mathrm{wH}[7], \mathrm{wL}[7]$, resultH[7], resultL[7]);

alu1 a8 (aH[8], aL[8], bH[8], bL[8], wH[7], wL[7], 0, 1, op [2], op[1:0], wH[8], wL[8], resultH[8], resultL[8]);

alu1 a9 (aH[9], aL[9], bH[9], bL[9], wH[8], wL[8], 0, 1, op [2], op[1:0], wH[9], wL[9], resultH[9], resultL[9]);

alu1 a10(aH[10], aL[10], bH[10], bL[10], wH[9], wL[9], 0, 1, op [2], op [1:0], wH[10], wL[10], resultH[10], resultL[10]);

alu1 a11(aH[11], aL[11], bH[11], bL[11], wH[10], wL[10], 0, 1, $\mathrm{op}[2], \mathrm{op}[1: 0], \mathrm{wH}[11], \mathrm{wL}[11], \operatorname{resultH}[11], \operatorname{resultL}[11])$;

alu1 a12 (aH[12], aL[12], bH[12], bL[12], wH[11], wL[11], 0, 1, $\mathrm{op}[2], \mathrm{op}[1: 0], \mathrm{wH}[12], \mathrm{wL}[12]$, resultH[12], resultL[12]);

alu1 a13(aH[13], aL[13], bH[13], bL[13], wH[12], wL[12], 0, 1, op [2], op [1:0], wH[13], wL[13], resultH[13], resultL[13]);

alu1 a14(aH[14], aL[14], bH[14], bL[14], wH[13], wL[13], 0, 1, op [2], op[1:0], wH[14], wL[14], resultH[14], resultL[14]);

alu1 a15 (aH[15], aL[15], bH[15], bL[15], wH[14], wL[14], 0, 1, op [2], op[1:0], wH[15], wL[15], resultH[15], resultL[15]);

alu1 a16 (aH[16], aL[16], bH[16], bL[16], wH[15], wL[15], 0, 1, $\mathrm{op}[2], \mathrm{op}[1: 0], \mathrm{wH}[16], \mathrm{wL}[16], \operatorname{resultH}[16]$, resultL[16]);

alu1 a17 (aH[17], aL[17], bH[17], bL[17], wH[16], wL[16], 0, 1, op [2], op[1:0], wH[17], wL[17], resultH[17], resultL[17]);

alu1 a18(aH[18], aL[18], bH[18], bL[18], wH[17], wL[17], 0, 1, op [2], op[1:0], wH[18], wL[18], resultH[18], resultL[18]);

alu1 a19(aH[19], aL[19], bH[19], bL[19], wH[18], wL[18], 0, 1, op [2], op[1:0], wH[19], wL[19], resultH[19], resultL[19]);

alu1 a20 (aH[20], aL[20], bH[20], bL[20], wH[19], wL[19], 0, 1, op [2], op [1:0], wH[20], wL[20], resultH[20], resultL[20]);

alu1 a21 (aH[21], aL[21], bH[21], bL[21], wH[20], wL[20], 0, 1, op [2], op [1:0], wH[21], wL[21], resultH[21], resultL[21]);

alu1 a22 (aH[22], aL[22], bH[22], bL[22], wH[21], wL[21], 0, 1, op [2], op [1:0], wH[22], wL[22], resultH[22], resultL[22]);

alu1 a23(aH[23], aL[23], bH[23], bL[23], wH[22], wL[22], 0, 1, op [2], op [1:0], wH[23], wL[23], resultH[23], resultL[23]); 
alu1 a24(aH[24], aL[24], bH[24], bL[24], wH[23], wL[23], 0, 1, op [2], op[1:0], $w H[24], w L[24]$, resultH[24], resultL[24]);

alu1 a25 (aH[25], aL[25], bH[25], bL[25], wH[24], wL[24], 0, 1, op [2], op [1:0], wH[25], wL[25], resultH[25], resultL[25]);

alu1 a26 (aH[26], aL[26], bH[26], bL[26], wH[25], wL[25], 0, 1, op [2], op[1:0], wH[26], wL[26], resultH[26], resultL[26]);

alu1 a27 (aH[27], aL[27], bH[27], bL[27], wH[26], wL[26], 0, 1, op [2], op [1:0], wH[27], wL[27], resultH[27], resultL[27]);

alu1 a28 (aH[28], aL[28], bH[28], bL[28], wH[27], wL[27], 0, 1, op [2], op[1:0], wH[28], wL[28], resultH[28], resultL[28]); alu1 a29 (aH[29], aL[29], bH[29], bL[29], wH[28], wL[28], 0, 1, op [2], op[1:0], wH[29], wL[29], resultH[29], resultL[29]); alu1 a30 (aH[30], aL[30], bH[30], bL[30], wH[29], wL[29], 0, 1, $\mathrm{op}[2], \mathrm{op}[1: 0], \mathrm{wH}[30], \mathrm{wL}[30], \operatorname{resultH}[30], \operatorname{resultL}[30])$; alu1end a31(aH[31], aL[31], bH[31], bL[31], wH[30], wL[30], 0, 1, op [2], op[1:0], lessH, lessL, resultH[31], resultL[31]);

or \#1 (notZero, resultH[31], resultH[30], resultH[29], resultH[28], resultH[27], resultH[26], resultH[25], resultH[24], resultH[23], resulth [22], resultH[21], resultH[20], resultH[19], resultH[18], resultH[17], resultH[16], resultH[15], resultH[14], resultH[13], resultH[12], resultH[11], resultH[10], resultH[9], resultH[8], resultH[7], resultH[6], resultH[5], resultH[4], resultH[3], resulth[2], resultH[1], resulth[0]);

endmodule // alu32

module adder32(aH, aL, bH, bL, resultH, resultL);

input $[31: 0] \mathrm{aH}, \mathrm{aL}, \mathrm{bH}, \mathrm{bL}$;

output [31:0] resultH, resultL;

wire [30:0] wH, wL;

wire aReady, bReady, inpReady;

xor \#1 (aReady, aH[0], aL[0]);

xor \#1 (bReady, bH[0], bL [0]);

and \#1 (inpReady, aReady, bReady);

adder $1 \mathrm{aO}(\mathrm{aH}[0], \mathrm{aL}[0], \mathrm{bH}[0], \mathrm{bL}[0], 0$, inpReady, $\mathrm{wH}[0], \mathrm{wL}[0]$, resulth [0], resultL[0]);

adder1 a1 (aH[1], aL[1], bH[1], bL[1], wH[0], wL[0], wH[1], wL[1], resultH[1], resultL[1]);

adder1 a2 (aH[2], aL[2], bH[2], bL[2], wH[1], wL[1], wH[2], wL[2], resultH[2], resultL[2]);

adder1 a3(aH[3], aL[3], bH[3], bL[3], wH[2], wL[2], wH[3], wL[3], resulth[3], resultL[3]);

adder1 a4(aH [4], aL [4], bH[4], bL [4], wH[3], wL [3], wH[4], wL[4], resulth [4], resultL[4]);

adder1 a5 (aH[5], aL[5], bH[5], bL[5], wH[4], wL[4], wH[5], wL[5], resultH[5], resultL[5]);

adder $1 \mathrm{a}$ (aH[6], aL[6], bH[6], bL[6], wH[5], wL[5], wH[6], wL[6] , resultH[6], resultL[6]); 
adder 1 a7 (aH[7], aL [7], bH[7], bL[7], wH[6], wL[6], wH[7] , wL[7] , resultH[7], resultL[7]);

adder1 a8(aH[8], aL[8], bH[8], bL[8], wH[7], wL[7], wH[8], wL[8], resultH[8], resultL[8]);

adder 1 a9(aH[9], aL[9], bH[9], bL[9], wH[8], wL[8], wH[9], wL[9], resulth [9], resultL[9]);

adder $1 \mathrm{a} 10(\mathrm{aH}[10], \mathrm{aL}[10], \mathrm{bH}[10], \mathrm{bL}[10], \mathrm{wH}[9], \mathrm{wL}[9], \mathrm{wH}[10], \mathrm{wL}[10]$, resultH[10], resultL[10]);

adder1 a11(aH[11], aL[11], bH[11], bL[11], wH[10], wL[10], wH[11], wL[11], resultH[11], resultL[11]);

adder 1 a12 (aH [12], aL [12], bH[12], bL [12], wH[11], wL [11], $w H[12], w L[12]$, resultH[12], resultL[12]);

adder $1 \mathrm{a} 13(\mathrm{aH}[13], \mathrm{aL}[13], \mathrm{bH}[13], \mathrm{bL}[13], \mathrm{wH}[12], \mathrm{wL}[12], \mathrm{wH}[13], \mathrm{wL}[13]$, resultH[13], resultL[13]);

adder 1 a14 (aH [14], aL [14], bH[14], bL [14], wH[13], wL [13], wH[14], wL[14], resultH[14], resultL[14]);

adder1 a15(aH[15], aL[15], bH[15], bL [15], wH[14], wL[14], wH[15], wL[15], resultH[15], resultL[15] );

adder1 a16 (aH[16], aL [16], bH[16], bL [16], wH[15], wL[15], wH[16], wL[16], resultH[16], resultL[16]);

adder1 a17 (aH[17], aL[17], bH[17], bL[17], wH[16], wL[16], wH[17], wL[17], resultH[17], resultL[17]);

adder1 a18 (aH[18], aL [18], bH[18], bL [18], wH[17], wL[17], wH[18], wL[18], resultH[18], resultL[18] );

adder1 a19 (aH[19], aL [19], bH[19], bL [19], wH[18], wL[18], wH[19], wL[19], resultH[19], resultL[19]);

adder 1 a20 (aH[20], aL [20], bH[20], bL [20], wH[19], wL[19], wH[20], wL[20], resultH[20], resultL[20]);

adder1 a21(aH[21], aL[21], bH[21], bL[21], wH[20], wL[20], wH[21], wL[21], resultH[21], resultL[21]);

adder1 a22(aH[22], aL[22], bH[22], bL [22], wH[21], wL[21], wH[22], wL[22], resultH[22], resultL[22]);

adder 1 a23 (aH [23], aL [23], bH[23], bL [23], wH[22], wL [22], wH[23], wL[23], resultH [23], resultL[23]);

adder 1 a24 (aH [24], aL [24], bH [24], bL [24], wH[23], wL [23], wH[24], wL[24], resultH[24], resultL[24]);

adder1 a25(aH[25], aL [25], bH[25], bL [25], wH[24], wL[24], wH[25], wL[25], resultH [25], resultL[25]);

adder1 a26(aH[26], aL[26], bH[26], bL[26], wH[25], wL[25], wH[26], wL[26], resultH[26], resultL[26]);

adder1 a27(aH[27], aL[27], bH[27], bL[27], wH[26], wL[26], wH[27], wL[27], resultH[27], resultL[27]);

adder1 a28(aH[28], aL [28], bH[28], bL[28], wH[27], wL[27], wH[28], wL[28] , resultH[28], resultL[28]);

adder 1 a29 (aH [29], aL [29], bH[29], bL [29], wH[28], wL [28], wH[29], wL[29], resultH[29], resultL [29] );

adder 1 a30 (aH[30], aL [30], bH[30], bL [30], wH[29], wL[29], wH[30], wL[30], resultH[30], resultL[30]);

adder1 a31(aH[31], aL[31], bH[31], bL[31], wH[30], wL[30], nowhereH, nowhereL, resultH[31], resultL[31]);

endmodule // adder32 


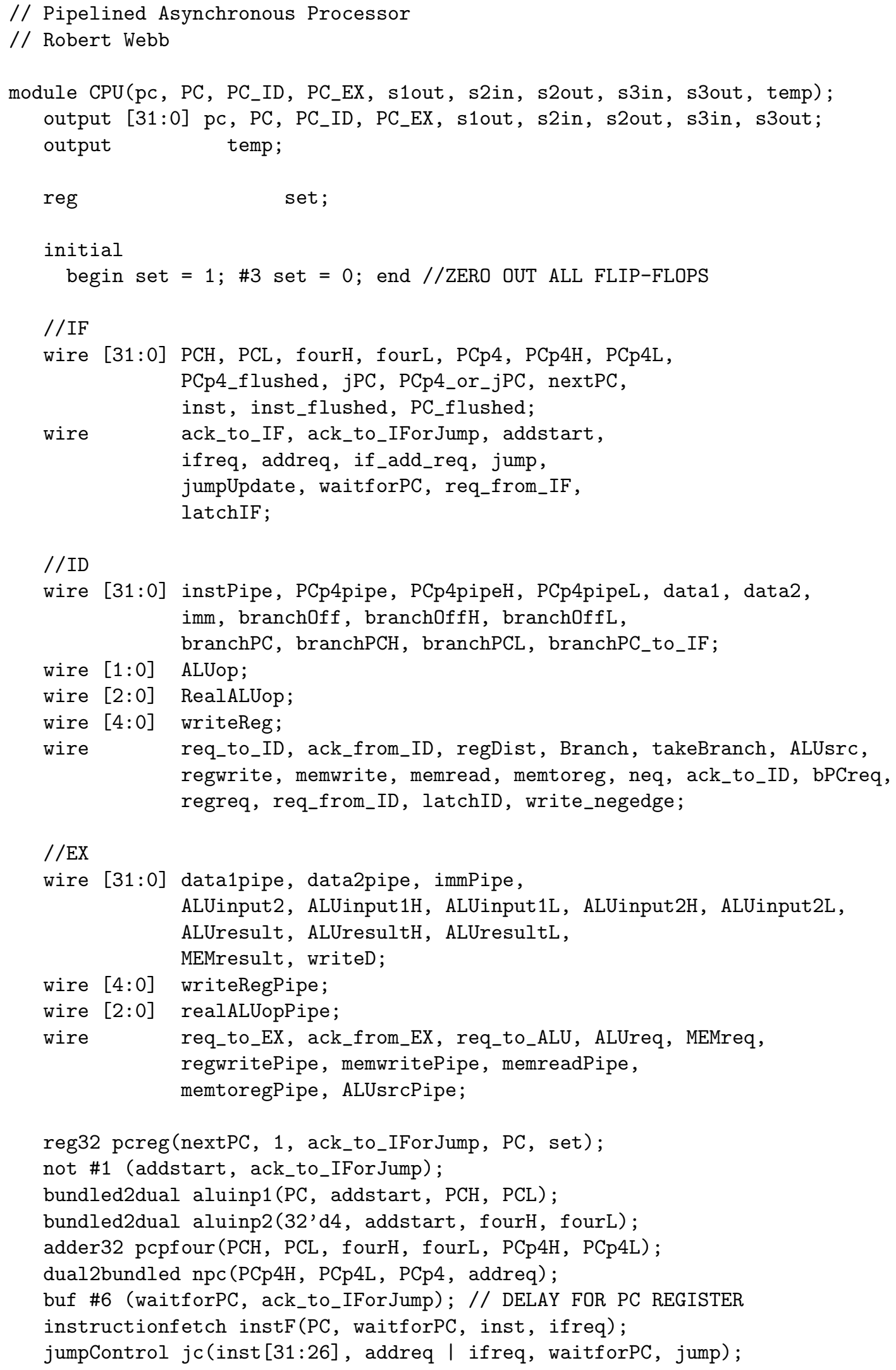




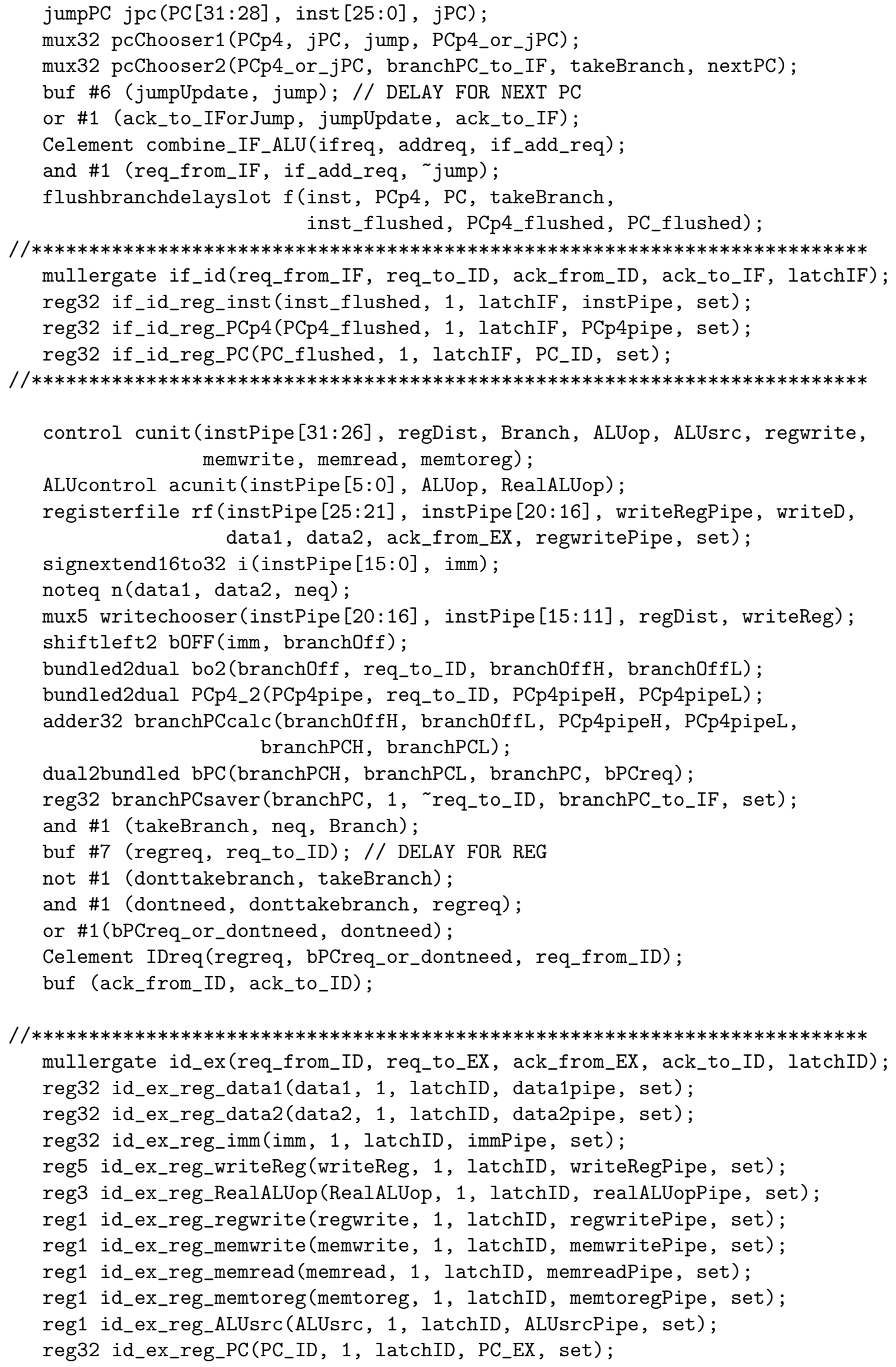




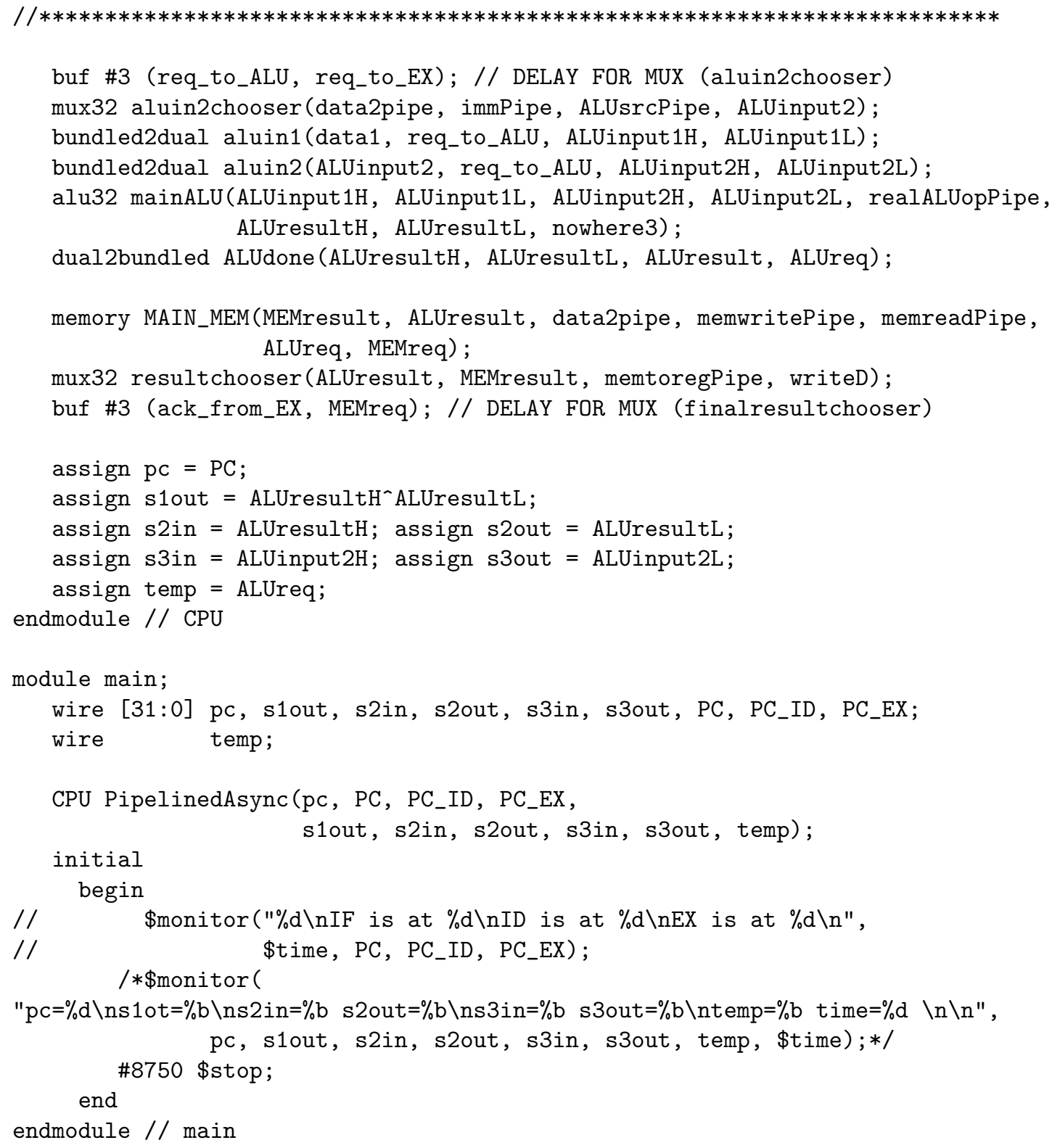




\section{Appendix D}

\section{Laboratory Exercises for students}

1. Using the single cycle asynchronous processor determine the delays of the global completion signal that are necessary to ensure correct operation.

2. Using the synchronous processor simulation and traditional boolean algebra convert the control unit to a simplified structural Verilog module. This lab is included because it is important for students to experience the difference between traditional logic design and the DIMS procedure.

3. Using the pipelined asynchronous processor convert the the behavioral control unit into a DIMS structural unit.

4. Using the pipelined asynchronous processor create a DIMS bit inverter to replace the behavioral bit inverter in the DIMS ALU. This requires that the control signals for the ALU be dual rail, which will be done in a later lab.

5. Using the pipelined asynchronous processor create a DIMS 4 input multiplexor to replace the behavioral multiplexor in the DIMS ALU. This also requires that the control signal be dual rail.

6. Using the pipelined asynchronous processor convert the remaining ALU controls to dual rail and integrate the changes from the previous labs. 